Supporting Information for

\title{
Copper(I) $\beta$-Boroalkyls from Alkene Insertion: Isolation and Rearrangement
}

\author{
David S. Laitar, Emily Y. Tsui, Joseph P. Sadighi* \\ Department of Chemistry, Massachusetts Institute of Technology \\ Cambridge, Massachusetts 02139
}

General Considerations. All synthetic manipulations were carried out using standard Schlenk techniques under an argon atmosphere, or in an Innovative Technologies glovebox under an atmosphere of purified nitrogen. Reactions were carried out in flame-dried glassware cooled under vacuum. Elemental analyses were performed by Desert Analytics, Tucson, AZ. Anhydrous toluene, hexanes, and tetrahydrofuran were purchased from Aldrich in $18-\mathrm{L}$ Pure-Pac ${ }^{\mathrm{TM}}$ solvent delivery kegs and sparged vigorously with argon for 40 minutes prior to first use. The solvents were further purified by passing them under argon pressure through two packed columns of neutral alumina and a third column packed with activated $4 \AA$ molecular sieves (for tetrahydrofuran) or through neutral alumina and copper(II) oxide (for toluene and hexanes). Benzene and pentane, anhydrous, were purchased from Aldrich in Sure-Seal ${ }^{\mathrm{TM}}$ bottles, and stored in a glove box over $4 \AA \AA$ molecular sieves. All non-dried solvents used were reagent grade or better.

NMR solvent $\mathrm{C}_{6} \mathrm{D}_{6}$ (Cambridge Isotope Laboratories) was dried over sodium/benzophenone, degassed by three freeze-pump-thaw cycles, and vacuum-transferred prior to use. ${ }^{1} \mathrm{H}$ NMR spectra were recorded on a Varian $300 \mathrm{MHz}$ instrument, with shifts reported relative to the residual solvent peak. ${ }^{19} \mathrm{~F}$ NMR spectra were recorded on a Varian $300 \mathrm{MHz}$ instrument, with shifts referenced to an external 
standard of $\mathrm{CFCl}_{3}$. ${ }^{11} \mathrm{~B} \mathrm{NMR}$ spectra were recorded on a Varian $500 \mathrm{MHz}$ instrument, with shifts referenced to an external standard of $0.5 \mathrm{M} \mathrm{BF}_{3}$ in diethyl ether $(0 \mathrm{ppm}) .{ }^{13} \mathrm{C}$ NMR spectra were recorded on a Varian $500 \mathrm{MHz}$ instrument, with shifts referenced relative to the solvent peak.

The starting compounds [1,3-bis(2,6-diisopropylphenyl)imidazol-2-ylidene]copper(I) (pinacolato)boryl ${ }^{1}$ and 4-dimethylaminostyrene ${ }^{2}$ were synthesized as described previously. Styrene (Lancaster), 4-fluorostyrene (Alfa Aesar), 4-methylstyrene (Alfa Aesar), 4-methoxystyrene (Alfa Aesar), 2-butyne (Aldrich), and cis-stilbene (Lancaster) were degassed prior to use. Ethylene (Airgas), trans-stilbene, trans-2-phenylvinyl(pinacol)boronate (Lancaster Synthesis) and trans-2-(ptolyl)vinyl(pinacol)boronate (Lancaster Synthesis) were used as received.

\section{General procedure for reaction of olefins and alkynes with (IPr)CuB(pin):}

In a glove box, a scintillation vial equipped with a magnetic stir bar was charged with (IPr)CuOt $\mathrm{Bu}$ $(0.300 \mathrm{~g}, 0.571 \mathrm{mmol})$ and bis(pinacolato)diboron $(0.146 \mathrm{~g}, 0.574 \mathrm{mmol}) . n$-Pentane $(5 \mathrm{~mL})$ was added, and the reaction mixture was stirred for 10 minutes. The appropriate olefin or alkyne $(0.628 \mathrm{mmol})$ was added via syringe and the reaction mixture was stirred for 20 minutes. The resulting white precipitate was collected by filtration.

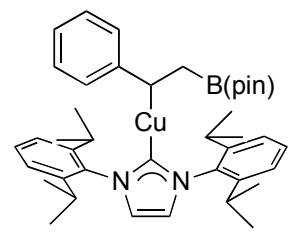

[1,3-Bis(2,6-diisopropylphenyl)imidazol-2-ylidene]copper(I) 1-phenyl-2-(pinacolboro)ethyl (1): The general procedure was followed to give the title compound $(0.354 \mathrm{~g}, 91 \%) .{ }^{1} \mathrm{H}$ NMR $\left(\mathrm{C}_{6} \mathrm{D}_{6}\right): \delta 7.23(\mathrm{t}, J$ $=7.8 \mathrm{~Hz}, 2 \mathrm{H}), 7.05(\mathrm{~m}, 6 \mathrm{H}), 7.01(\mathrm{~d}, J=7.6 \mathrm{~Hz}, 2 \mathrm{H}), 6.82(\mathrm{~d}, J=7.6 \mathrm{~Hz}, 2 \mathrm{H}), 6.74(\mathrm{t}, J=7.1 \mathrm{~Hz}, 1$ H), $6.20(\mathrm{~s}, 2 \mathrm{H}), 2.57(\mathrm{t}, J=7.8 \mathrm{~Hz}, 1 \mathrm{H}), 2.49(\mathrm{~m}, 4 \mathrm{H}), 1.64(\mathrm{~d}, J=7.8 \mathrm{~Hz}, 2 \mathrm{H}), 1.30(\mathrm{~d}, J=6.9 \mathrm{~Hz}$ $6 \mathrm{H}), 1.28(\mathrm{~d}, J=6.9 \mathrm{~Hz}, 6 \mathrm{H}), 1.068(\mathrm{~d}, J=6.9 \mathrm{~Hz}, 6 \mathrm{H}), 1.066(\mathrm{~d}, J=6.9 \mathrm{~Hz}, 6 \mathrm{H}), 0.984(\mathrm{~s}, 6 \mathrm{H})$, $0.979(\mathrm{~s}, 6 \mathrm{H}) .{ }^{13} \mathrm{C}$ NMR $\left(\mathrm{C}_{6} \mathrm{D}_{6}\right): \delta 186.0\left(\mathrm{~N}_{2} \mathrm{CCu}\right), 159.5$ (ipso- $\left.\mathrm{C}_{6} \mathrm{H}_{5}\right), 146.0(o-\mathrm{Ar}), 145.9(o-\mathrm{Ar}), 135.7$ 
(ipso-Ar), 130.7 (p-Ar), $128.0\left(m-\mathrm{C}_{6} \mathrm{H}_{5}\right), 125.1\left(o-\mathrm{C}_{6} \mathrm{H}_{5}\right), 124.4(m-\mathrm{Ar}), 122.3(\mathrm{~N} \underline{\mathrm{CH}}), 117.4\left(p-\mathrm{C}_{6} \mathrm{H}_{5}\right)$, $82.1\left(\mathrm{~B}\left(\mathrm{OC}\left(\mathrm{CH}_{3}\right)_{2}\right)_{2}, 30.7\left(\mathrm{CuC}_{\alpha}\right), 29.3\left(\underline{\mathrm{CH}}\left(\mathrm{CH}_{3}\right)_{2}\right), 25.5\left(\mathrm{~B}\left(\mathrm{OC}(\underline{\mathrm{CH}})_{3}\right)_{2}\right)_{2}, 25.2\left(\mathrm{CH}\left(\underline{\mathrm{CH}}_{3}\right)_{2}\right), 24.1\right.$ $\left(\mathrm{CH}\left(\mathrm{CH}_{3}\right)_{2}\right), 16.0$ (br., $\mathrm{CH}_{2} \mathrm{~B}($ pin) $\left.)\right) .{ }^{11} \mathrm{~B} \mathrm{NMR}\left(\mathrm{C}_{6} \mathrm{D}_{6}\right): \delta 34.7$.

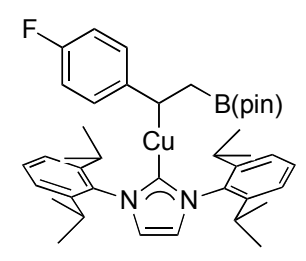

\section{[1,3-Bis(2,6-diisopropylphenyl)imidazol-2-ylidene]copper(I) 1-(4-fluorophenyl)-2-(pinacolboro)-}

ethyl: The general procedure was followed to give the title compound $(0.280 \mathrm{~g}, 70 \%) .{ }^{1} \mathrm{H}$ NMR $\left(\mathrm{C}_{6} \mathrm{D}_{6}\right): \delta 7.22(\mathrm{t}, J=7.8 \mathrm{~Hz}, 2 \mathrm{H}), 7.03(\mathrm{~d}, J=7.6 \mathrm{~Hz}, 2 \mathrm{H}), 7.01(\mathrm{~d}, J=7.6 \mathrm{~Hz}, 2 \mathrm{H}), 6.75(\mathrm{~m}, 2 \mathrm{H})$, $6.63(\mathrm{~m}, 2 \mathrm{H}), 6.17$ (s, $2 \mathrm{H}), 2.47$ (m, 5 H), 1.58 (m, $2 \mathrm{H}), 1.28$ (d, J= $7.0 \mathrm{~Hz}, 6 \mathrm{H}), 1.25$ (d, $J=7.0 \mathrm{~Hz}$, $6 \mathrm{H}), 1.06(\mathrm{~d}, J=7.0 \mathrm{~Hz}, 6 \mathrm{H}), 1.05(\mathrm{~d}, J=7.0 \mathrm{~Hz}, 6 \mathrm{H}), 0.98(\mathrm{~s}, 12 \mathrm{H}) .{ }^{13} \mathrm{C} \mathrm{NMR}\left(\mathrm{C}_{6} \mathrm{D}_{6}\right): \delta 186.0$ $\left(\mathrm{N}_{2} \underline{\mathrm{CCu}}\right), 157.5$ (d, J $\left.J_{C-F}=232 \mathrm{~Hz}, \underline{\mathrm{C}}-\mathrm{F}\right), 155.1$ (ipso- $\left.\mathrm{C}_{6} \mathrm{H}_{4} \mathrm{~F}\right), 145.9$ (o-Ar), 135.7 (ipso-Ar), 130.7 (pAr), $\left.125.3\left(\mathrm{~d}, J_{C-F}=6.3 \mathrm{~Hz}\right), 124.4(m-\mathrm{Ar}), 122.4(\mathrm{~N} \underline{\mathrm{CH}}), 114.3\left(\mathrm{~d}, J_{C-F}=20 \mathrm{~Hz}\right)\right), 82.1\left(\mathrm{~B}\left(\mathrm{O} \underline{\mathrm{C}}\left(\mathrm{CH}_{3}\right)_{2}\right)_{2}\right.$, $29.4\left(\mathrm{Cu}_{\alpha}\right), 29.2\left(\underline{\mathrm{CH}}\left(\mathrm{CH}_{3}\right)_{2}\right), 25.5,25.4,24.0\left(\mathrm{CH}\left(\underline{\mathrm{CH}}_{3}\right)_{2}\right), 16.5$ (br., $\underline{\mathrm{CH}}_{2} \mathrm{~B}($ pin $\left.)\right) .{ }^{19} \mathrm{~F}$ NMR $\left(\mathrm{C}_{6} \mathrm{D}_{6}\right): \delta$ -130.2. ${ }^{11} \mathrm{~B}$ NMR $\left(\mathrm{C}_{6} \mathrm{D}_{6}\right): \delta 34.7$.

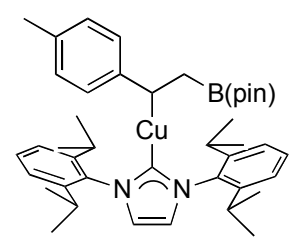

[1,3-Bis(2,6-diisopropylphenyl)imidazol-2-ylidene]copper(I) 1-(4-methylphenyl)-2-(pinacolboro)ethyl: The general procedure was followed to give the title compound $(0.350 \mathrm{~g}, 88 \%) .{ }^{1} \mathrm{H}$ NMR $\left(\mathrm{C}_{6} \mathrm{D}_{6}\right)$ : $\delta 7.23$ (t, $J=7.7 \mathrm{~Hz}, 2 \mathrm{H}), 7.05(\mathrm{~m}, 4 \mathrm{H}), 6.84(\mathrm{~d}, J=8.0 \mathrm{~Hz}, 2 \mathrm{H}), 6.75(\mathrm{~d}, J=8.2 \mathrm{~Hz}, 2 \mathrm{H}), 6.20$ (s, 2 H), $2.50(\mathrm{~m}, 5 \mathrm{H}), 2.35(\mathrm{~s}, 3 \mathrm{H}), 1.65(\mathrm{~d}, J=8.2 \mathrm{~Hz}, 2 \mathrm{H}), 1.30(\mathrm{~m}, 12 \mathrm{H}), 1.07(\mathrm{~d}, J=6.9 \mathrm{~Hz}, 12 \mathrm{H})$, $0.99(\mathrm{~s}, 12 \mathrm{H}) .{ }^{13} \mathrm{C}$ NMR $\left(\mathrm{C}_{6} \mathrm{D}_{6}\right): \delta 186.1\left(\mathrm{~N}_{2} \underline{\mathrm{CCu}}\right), 156.2,145.9,135.8,130.6,128.7,125.1,124.9$, 124.4, 122.4, 82.0, $29.9\left(\underline{\mathrm{C}}_{\alpha} \mathrm{Cu}\right), 29.3,25.6,25.4,25.2,24.1,21.6\left(p-\mathrm{CH}_{3}\right), 16.3$ (br., $\underline{\mathrm{C}}_{2} \mathrm{~B}($ pin) $)$. ${ }^{11} \mathrm{~B}$ 
$\operatorname{NMR}\left(\mathrm{C}_{6} \mathrm{D}_{6}\right): \delta 35.5$.

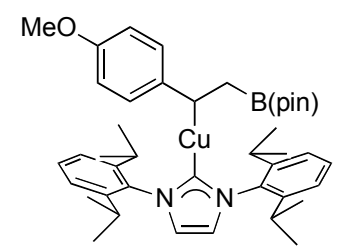

[1,3-Bis(2,6-diisopropylphenyl)imidazol-2-ylidene]copper(I) 1-(4-methoxyphenyl)-2-(pinacolboro)-

ethyl: The general procedure was followed to give the title compound $(0.374 \mathrm{~g}, 92 \%) .{ }^{1} \mathrm{H}$ NMR $\left(\mathrm{C}_{6} \mathrm{D}_{6}\right): \delta 7.25(\mathrm{t}, J=7.8 \mathrm{~Hz}, 2 \mathrm{H}), 7.06(\mathrm{~m}, 4 \mathrm{H}), 6.71(\mathrm{~m}, 4 \mathrm{H}), 6.19(\mathrm{~s}, 2 \mathrm{H}), 3.58(\mathrm{~s}, 3 \mathrm{H}), 2.50(\mathrm{~m}, 5$ H), 1.65 (m, 2 H), 1.32 (d, J=6.9 Hz, $6 \mathrm{H}), 1.30$ (d, $J=6.9 \mathrm{~Hz}, 6 \mathrm{H}), 1.07$ (d, $J=6.9 \mathrm{~Hz}, 12 \mathrm{H}), 0.99$ (s, $12 \mathrm{H}) .{ }^{13} \mathrm{C}$ NMR $\left(\mathrm{C}_{6} \mathrm{D}_{6}\right): \delta 186.1\left(\mathrm{~N}_{2} \mathrm{CCu}\right), 153.1,152.0,145.9,135.7,130.6,125.5,124.4,122.3,114.2$, 82.0, $55.7\left(\mathrm{OCH}_{3}\right), 29.3,28.9\left(\underline{\mathrm{C}}_{a} \mathrm{Cu}\right), 25.6,25.4,25.2,24.1,16.8$ (br., $\underline{\mathrm{CH}}_{2} \mathrm{~B}($ pin) $) .{ }^{11} \mathrm{~B} \mathrm{NMR}\left(\mathrm{C}_{6} \mathrm{D}_{6}\right): \delta$ 34.8 .

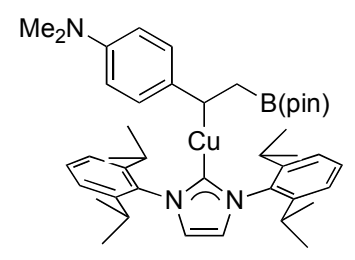

[1,3-Bis(2,6-diisopropylphenyl)imidazol-2-ylidene]copper(I)

1-(4-dimethylaminophenyl)-2-

(pinacolboro)ethyl: The general procedure was followed to give the title compound $(0.368 \mathrm{~g}, 89 \%) .{ }^{1} \mathrm{H}$ $\operatorname{NMR}\left(\mathrm{C}_{6} \mathrm{D}_{6}\right): \delta 7.23(\mathrm{t}, J=7.7 \mathrm{~Hz}, 2 \mathrm{H}), 7.08(\mathrm{~m}, 4 \mathrm{H}), 6.76(\mathrm{~d}, J=8.5 \mathrm{~Hz}, 2 \mathrm{H}), 6.62(\mathrm{~d}, J=8.5 \mathrm{~Hz}, 2$ H), $6.20(\mathrm{~s}, 2 \mathrm{H}), 2.76(\mathrm{~s}, 6 \mathrm{H}), 2.52(\mathrm{~m}, 5 \mathrm{H}), 1.68(\mathrm{~m}, 2 \mathrm{H}), 1.33(\mathrm{~d}, J=6.9 \mathrm{~Hz}, 6 \mathrm{H}), 1.32(\mathrm{~d}, J=6.9$ $\mathrm{Hz}, 6 \mathrm{H}), 1.08$ (d, J=6.9 Hz, $12 \mathrm{H}), 1.00(\mathrm{~s}, 12 \mathrm{H}) .{ }^{13} \mathrm{C} \mathrm{NMR}\left(\mathrm{C}_{6} \mathrm{D}_{6}\right): \delta 186.4\left(\mathrm{~N}_{2} \underline{\mathrm{CCu}}\right), 150.4,146.0$, $144.5,135.8,130.6,125.5,124.4,122.3,116.2,82.0,43.4\left(\mathrm{~N}\left(\underline{\mathrm{CH}}_{3}\right)_{2}\right), 29.3,28.8\left(\underline{\mathrm{C}}_{\alpha} \mathrm{Cu}\right), 25.6,25.4$, 25.3, 24.1, 16.8 (br., $\underline{\mathrm{CH}}_{2} \mathrm{~B}\left(\right.$ pin) )). ${ }^{11} \mathrm{~B}$ NMR $\left(\mathrm{C}_{6} \mathrm{D}_{6}\right)$ : $\delta 35.5$.

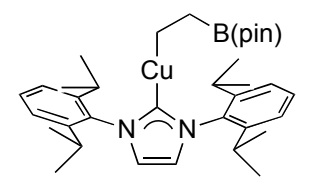


[1,3-Bis(2,6-diisopropylphenyl)imidazol-2-ylidene]copper(I) 2-(pinacolboro)ethyl: The general procedure was followed except that the pentane suspension of (IPr)CuB(pin) was degassed on a Schlenk line, and ethylene (1 $1 \mathrm{~atm})$ was added to give the title compound $(0.297 \mathrm{~g}, 86 \%) .{ }^{1} \mathrm{H}$ NMR $\left(\mathrm{C}_{6} \mathrm{D}_{6}\right): \delta 7.21$ (t, $J=7.7 \mathrm{~Hz}, 2 \mathrm{H}), 7.07$ (m, $J=7.4 \mathrm{~Hz}, 4 \mathrm{H}), 6.23$ (s, $2 \mathrm{H}), 2.64$ (sept., $J=6.9 \mathrm{~Hz}, 4 \mathrm{H}), 1.43$ (d, $J=6.9$ $\mathrm{Hz}, 12 \mathrm{H}), 1.21(\mathrm{~m}, 2 \mathrm{H}), 1.10(\mathrm{~d}, J=6.9 \mathrm{~Hz}, 12 \mathrm{H}), 1.05(\mathrm{~s}, 12 \mathrm{H}), 0.44(\mathrm{~m}, 2 \mathrm{H}) .{ }^{13} \mathrm{C}$ NMR $\left(\mathrm{C}_{6} \mathrm{D}_{6}\right): \delta$ $186.7(\mathrm{NCCu}), 146.2,135.8,130.6,124.4,122.1,81.7,29.3,25.4,24.1,10.3\left(\mathrm{CH}_{2} \mathrm{~B}(\operatorname{pin}), 2.4\left(\mathrm{CuCH}_{2}\right)\right.$. ${ }^{11} \mathrm{~B} \operatorname{NMR}\left(\mathrm{C}_{6} \mathrm{D}_{6}\right): \delta 34.5$.

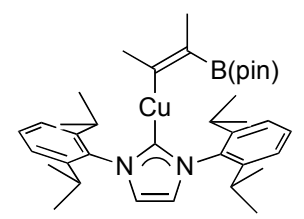

[1,3-Bis(2,6-diisopropylphenyl)imidazol-2-ylidene]copper(I) (Z)-3-(pinacolatoboro)-2-butenyl: The general procedure was followed, except that a large excess of 3-butyne $(\sim 0.25 \mathrm{~mL})$ was used due to its high volatility, to give the title compound $(0.326 \mathrm{~g}, 90 \%) .{ }^{1} \mathrm{H}$ NMR $\left(\mathrm{C}_{6} \mathrm{D}_{6}\right): \delta 7.27$ (t, $\left.J=7.8 \mathrm{~Hz}, 2 \mathrm{H}\right)$, 7.14 (d, $J=7.6 \mathrm{~Hz}, 2 \mathrm{H}), 6.26$ (s, $2 \mathrm{H}), 2.68$ (sept, $J=6.9 \mathrm{~Hz}, 4 \mathrm{H}), 2.22$ (s, $3 \mathrm{H}), 1.74$ (s, $3 \mathrm{H}), 1.45$ (d, $J=6.9 \mathrm{~Hz}, 12 \mathrm{H}), 1.13(\mathrm{~d}, J=6.9 \mathrm{~Hz}, 12 \mathrm{H}), 0.96(\mathrm{~s}, 12 \mathrm{H}) .{ }^{13} \mathrm{C}$ NMR $\left(\mathrm{C}_{6} \mathrm{D}_{6}\right): \delta 192.8\left(\mathrm{C}_{\alpha}\right), 187.2$ $\left(\mathrm{N}_{2} \underline{\mathrm{CCu}}\right), 146.3,136.6,132.9$ (br., $\underline{\mathrm{CB}}($ pin)) 130.6, 124.6, 122.7, 81.5, 29.4, 25.6, 25.4, 25.2, 24.0, 15.5. ${ }^{11} \mathrm{~B} \operatorname{NMR}\left(\mathrm{C}_{6} \mathrm{D}_{6}\right): \delta 30.4$.

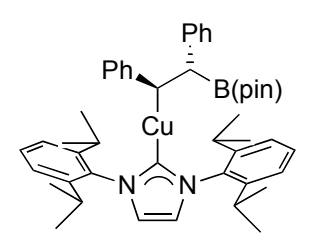

trans-Stilbene insertion: The general procedure was followed, except the reaction mixture was stirred for $15 \mathrm{hrs}$, to give the title compound $(0.351 \mathrm{~g}, 81 \%) .{ }^{1} \mathrm{H}$ NMR $\left(\mathrm{C}_{6} \mathrm{D}_{6}\right): \delta 7.51(\mathrm{~d}, J=6.9 \mathrm{~Hz}, 2 \mathrm{H}), 7.21$ (t, $J=7.7 \mathrm{~Hz}, 2 \mathrm{H}), 7.06-7.01(6 \mathrm{H}), 6.85(\mathrm{t}, J=7.4 \mathrm{~Hz}, 1 \mathrm{H}), 6.81$ (t, $J=7.2 \mathrm{~Hz}, 2 \mathrm{H}), 6.71(\mathrm{~d}, J=7.2$ Hz, $2 \mathrm{H}), 6.55$ (t, $J=7.2 \mathrm{~Hz}, 1 \mathrm{H}), 6.19(\mathrm{~s}, 2 \mathrm{H}), 3.54(\mathrm{~d}, J=11.6 \mathrm{~Hz}, 1 \mathrm{H}), 3.12(\mathrm{~d}, J=11.6 \mathrm{~Hz}, 1 \mathrm{H})$, 
$2.57(\mathrm{~m}, 4 \mathrm{H}), 1.36(\mathrm{~d}, J=6.9 \mathrm{~Hz}, 6 \mathrm{H}), 1.33(\mathrm{~d}, J=6.9 \mathrm{~Hz}, 6 \mathrm{H}), 1.07(\mathrm{~d}, J=6.9 \mathrm{~Hz}, 12 \mathrm{H}), 0.91(\mathrm{~s}, 6$ $\mathrm{H}), 0.86(\mathrm{~s}, 6 \mathrm{H}) .{ }^{13} \mathrm{C} \mathrm{NMR}\left(\mathrm{C}_{6} \mathrm{D}_{6}\right): \delta 185.8,155.2,147.5,145.70,145.68,135.9,130.7,129.8,128.0$, $127.9,126.4,124.6,124.5,124.1,122.7,117.7,82.3,37.3,36.2,29.31,29.27,25.6,25.3,25.2,25.0$, 24.2, 24.15. ${ }^{11} \mathrm{~B} \mathrm{NMR}\left(\mathrm{C}_{6} \mathrm{D}_{6}\right): \delta 34.0$.

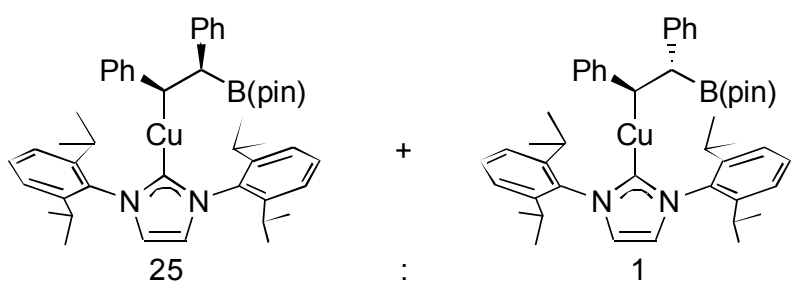

cis-Stilbene insertion product: The general procedure was followed, except 2 equiv of cis-stilbene (203 $\mu \mathrm{L}, 1.14 \mathrm{mmol}$ ) was used and the mixture was stirred for 15 hours, to give a mixture of syn and anti insertion products $(0.330 \mathrm{~g}, 76 \%) .{ }^{1} \mathrm{H} \mathrm{NMR}\left(\mathrm{C}_{6} \mathrm{D}_{6}\right): \delta 7.36(\mathrm{~d}, J=6.9 \mathrm{~Hz}, 2 \mathrm{H}), 7.24(\mathrm{t}, J=7.8 \mathrm{~Hz}$, $2 \mathrm{H}), 7.07-7.00(9 \mathrm{H}), 6.89(\mathrm{~d}, J=7.2 \mathrm{~Hz}, 2 \mathrm{H}), 6.77(\mathrm{t}, J=7.1 \mathrm{~Hz}, 1 \mathrm{H}), 6.71(\mathrm{~d}, J=7.2 \mathrm{~Hz}, 2 \mathrm{H}), 6.55$ (t, $J=7.2 \mathrm{~Hz}, 1 \mathrm{H}), 6.13(\mathrm{~s}, 2 \mathrm{H}), 3.30(\mathrm{~d}, J=12.5 \mathrm{~Hz}, 1 \mathrm{H}), 2.82(\mathrm{~d}, J=12.5 \mathrm{~Hz}, 1 \mathrm{H}), 2.40$ (sept., $J=$ $6.9 \mathrm{~Hz}, 2 \mathrm{H}), 2.34$ (sept., $J=6.9 \mathrm{~Hz}, 2 \mathrm{H}), 1.16$ (d, $J=6.9 \mathrm{~Hz}, 6 \mathrm{H}), 1.00-1.05$ (18 H), 0.87 (s, $6 \mathrm{H})$, $0.82(\mathrm{~s}, 6 \mathrm{H}) .{ }^{13} \mathrm{C} \mathrm{NMR}\left(\mathrm{C}_{6} \mathrm{D}_{6}\right): \delta 185.6,156.8,148.6,145.90,145.8,135.6,130.5,129.2,128.3,128.1$, $125.7,124.5,124.4,123.9,122.2,117.9,82.5,43.2,36.8,29.2,29.17,25.2,25.1,25.0,24.9,24.2,23.9$. ${ }^{11} \mathrm{~B}$ NMR $\left(\mathrm{C}_{6} \mathrm{D}_{6}\right): \delta 34.7$.

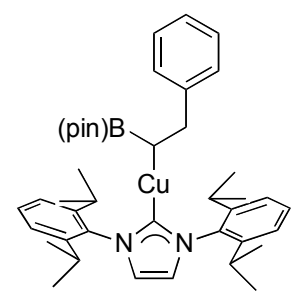

\section{[1,3-Bis(2,6-diisopropylphenyl)imidazol-2-ylidene $] \operatorname{copper(I)~}$}

(1-pinacolboro)-2-phenylethyl

(2a):

Method A: In a glovebox, a resealable Schlenk flask equipped with a magnetic stir bar was charged with $1(0.250 \mathrm{~g}, 0.356 \mathrm{mmol})$ and benzene $(10 \mathrm{~mL})$. The flask was sealed with a teflon stopcock, taken 
out of the glovebox and heated at $70{ }^{\circ} \mathrm{C}$ for 24 hours. The flask was allowed to cool and taken into the glovebox, and the reaction mixture was filtered through celite. The solution was then concentrated in vacuo and the resulting solid was washed with pentane to afford the title compound as an off-white solid (0.136 g, 54\%).

Method B: In a glove box, a vial is charged with (IPr) $\mathrm{CuOt} t \mathrm{Bu}(0.300 \mathrm{~g}, 0.571 \mathrm{mmol})$ and pentane (5 $\mathrm{mL})$. Triethoxysilane was added $(116 \mu \mathrm{L}, 0.628 \mathrm{mmol})$ and the mixture was stirred for 5 minutes. A solution of trans-2-phenylvinyl(pinacol)boronate $(0.144 \mathrm{~g}, 0.628 \mathrm{mmol})$ in $n$-pentane $(3 \mathrm{~mL})$ was then added and the mixture was stirred for 1 hour. The resulting white precipitate was collected by filtration to yield the title complex (354 mg, 91\%). ${ }^{1} \mathrm{H}$ NMR $\left(\mathrm{C}_{6} \mathrm{D}_{6}\right): \delta 7.45(\mathrm{~d}, J=6.9 \mathrm{~Hz}, 2 \mathrm{H}), 7.24-7.18(4 \mathrm{H})$, $7.08(4 \mathrm{H}), 7.04(\mathrm{t}, J=6.1 \mathrm{~Hz}, 1 \mathrm{H}), 6.23(\mathrm{~s}, 2 \mathrm{H}), 3.38(\mathrm{dd}, J=14.5 \mathrm{~Hz}, J=11.3 \mathrm{~Hz}, 1 \mathrm{H}), 2.80(\mathrm{dd}, J=$ $14.5 \mathrm{~Hz}, J=4.1 \mathrm{~Hz}, 1 \mathrm{H}), 2.59$ (sept, $J=6.9 \mathrm{~Hz}, 4 \mathrm{H}), 1.43$ (d, $J=6.9 \mathrm{~Hz}, 6 \mathrm{H}), 1.42$ (d, $J=6.9 \mathrm{~Hz}, 6$ H), $1.09(\mathrm{~d}, J=6.9 \mathrm{~Hz}, 12 \mathrm{H}), 1.03(\mathrm{~s}, 6 \mathrm{H}), 0.98(7 \mathrm{H})$. The proton on $\mathrm{C}_{\alpha}$ was located by 2D NMR spectroscopy (HSQC) and determined to be accidentally degenerate with a pinacol- $\mathrm{CH}_{3}$ resonance at 0.98 ppm. ${ }^{13} \mathrm{C}$ NMR $\left(\mathrm{C}_{6} \mathrm{D}_{6}\right): \delta 184.9\left(\mathrm{~N}_{2} \underline{\mathrm{CCu}}\right), 152.2,146.2,135.7,130.8,128.7,128.2,124.6,124.5$, 124.4, 122.5, 80.2, 34.9, 29.3, 29.2, 25.7, 25.5, 25.4, 24.2, 24.1, 18.1. ${ }^{11} \mathrm{~B}$ NMR $\left(\mathrm{C}_{6} \mathrm{D}_{6}\right): \delta 33.4$.

\section{Thermal Rearrangement of 1 in the presence of trans-2-(p-tolyl)vinyl(pinacol)boronate:}

In a glove box, $1(0.040 \mathrm{~g}, 0.0585 \mathrm{mmol})$ and trans-2-( $p$-tolyl)vinyl(pinacol)boronate $(0.021 \mathrm{~g}, 0.0878$ mmol) were dissolved in $\mathrm{C}_{6} \mathrm{D}_{6}(0.7 \mathrm{~mL})$ and the solution was added to an NMR tube. The NMR tube was sealed with a Teflon stopcock and heated at $70{ }^{\circ} \mathrm{C}$ for 24 hours. The tube was then cooled to room

temperature and inserted into an NMR spectrometer. The ${ }^{1} \mathrm{H}$ NMR spectrum indicated that a mixture of $\mathbf{2 a}$ and $\mathbf{2 b}$ had formed.

\section{Reversible $\beta$-Hydride Elimination Experiment:}

In a glove box, $2 \mathrm{a}(0.040 \mathrm{~g}, 0.0585 \mathrm{mmol})$ and trans-2-( $p$-tolyl)vinyl(pinacol)boronate $(0.020 \mathrm{~g}, 0.0878$ mmol) were dissolved in $\mathrm{C}_{6} \mathrm{D}_{6}(0.7 \mathrm{~mL})$ and the resulting solution was added to an NMR tube. The 
NMR tube was sealed with a Teflon stopcock and heated at $70{ }^{\circ} \mathrm{C}$ for 24 hours. The tube was then cooled to room temperature and inserted into an NMR spectrometer. The ${ }^{1} \mathrm{H}$ NMR spectrum indicated a 1:9 ratio of $\mathbf{2 b}$ to starting complex $\mathbf{2 a}$.

\section{Hammett Substituent Effect Study:}

\begin{tabular}{|c|c|c|}
\hline $\mathrm{X}$ & $\sigma_{\mathrm{p}}$ & $\mathrm{k}_{\mathrm{x}} / \mathrm{k}_{\mathrm{H}}$ \\
\hline $\mathrm{F}$ & 0.06 & $0.79(1)$ \\
\hline $\mathrm{Me}$ & -0.17 & $0.27(1)$ \\
\hline $\mathrm{OMe}$ & -0.27 & $0.068(15)$ \\
\hline $\mathrm{NMe}_{2}$ & -0.83 & $0.016(2)$ \\
\hline
\end{tabular}

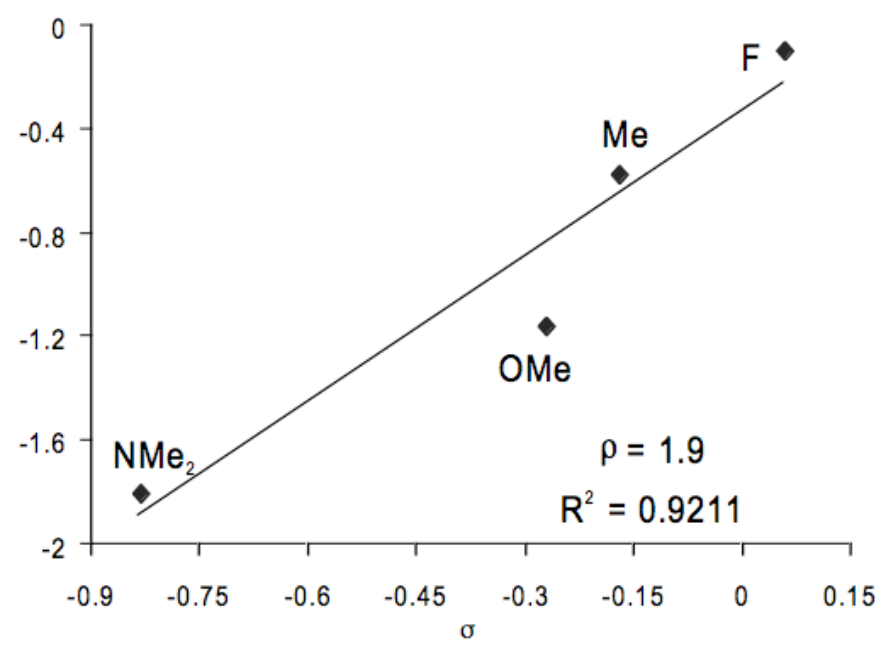

In a glove box, styrene $(200 \mu \mathrm{L}, 1.73 \mathrm{mmol})$ and a 4 -substituted styrene $(1.73 \mathrm{mmol})$ were added to a $10 \mathrm{~mL}$ volumetric flask. Benzene was added such that the total volume of solution was $10 \mathrm{~mL}$. After homogenization, the solution $(500 \mu \mathrm{L}, 0.0863 \mathrm{mmol}$ of each olefin) was rapidly added to a benzene solution of (IPr)CuB(pin) $(0.025 \mathrm{~g}, 0.0431 \mathrm{mmol}$ dissolved in $2 \mathrm{~mL})$. After stirring for 10 minutes, the solvent was removed in vacuo and the resulting solid was dissolved in $\mathrm{C}_{6} \mathrm{D}_{6}(0.7 \mathrm{~mL})$. The ratios of the insertion products were measured by integration of ${ }^{1} \mathrm{H}$ NMR spectra as follows: For styrene vs 4-fluorostyrene, the $\beta$-protons ((IPr) $\left.\mathrm{CuCH}(\mathrm{Ar}) \mathrm{C}_{2} \mathrm{~B}(\mathrm{pin})\right)$ of each complex were compared directly. For the other styrenes, the $\beta$-protons of the insertion products overlapped, and the ratios were measured indirectly by integration of the $\beta$-protons of both complexes compared with a distinct resonance of the 4substituted styrene insertion product after simple arithmetic. Specifically, the $\underline{\mathrm{CH}}_{3}, \mathrm{OCH}_{3}, \mathrm{~N}\left(\mathrm{CH}_{3}\right)_{2}$ peaks of the 4-substituted styrene insertion products were compared with the $\beta$-protons of both complexes. 


\section{Note on characterization of alkene insertion products}

Attempted elemental analyses of the insertion products did not give satisfactory results, even for samples in which no impurities were evident by ${ }^{1} \mathrm{H}$ or ${ }^{13} \mathrm{C}$ NMR spectroscopy. We attribute this to the very high air-sensitivity of these complexes, and to their perhaps imperfect thermal stability over the timeframe required to send analytical samples. Attempts at melting point determination for the insertion products of styrene, $p$-fluorostyrene, $p$-dimethylaminostyrene, and trans-stilbene, as well as for the $\alpha$ boroalkyl complex 2a, were unsuccessful: all these complexes rapidly turned orange, then black, in color on heating to temperatures much over $100{ }^{\circ} \mathrm{C}$ (the temperatures varied according to sample and heating rate). The following pages (S10-S29) display the ${ }^{1} \mathrm{H}$ and ${ }^{13} \mathrm{C}$ NMR spectra of all isolated complexes reported in the manuscript, so that the reader may judge their purity. Based on these spectra, we judge the reported complexes to be over $90 \%$ pure. Reasonably extensive efforts to purify them further have proven self-defeating due to the sensitive nature of these products. 


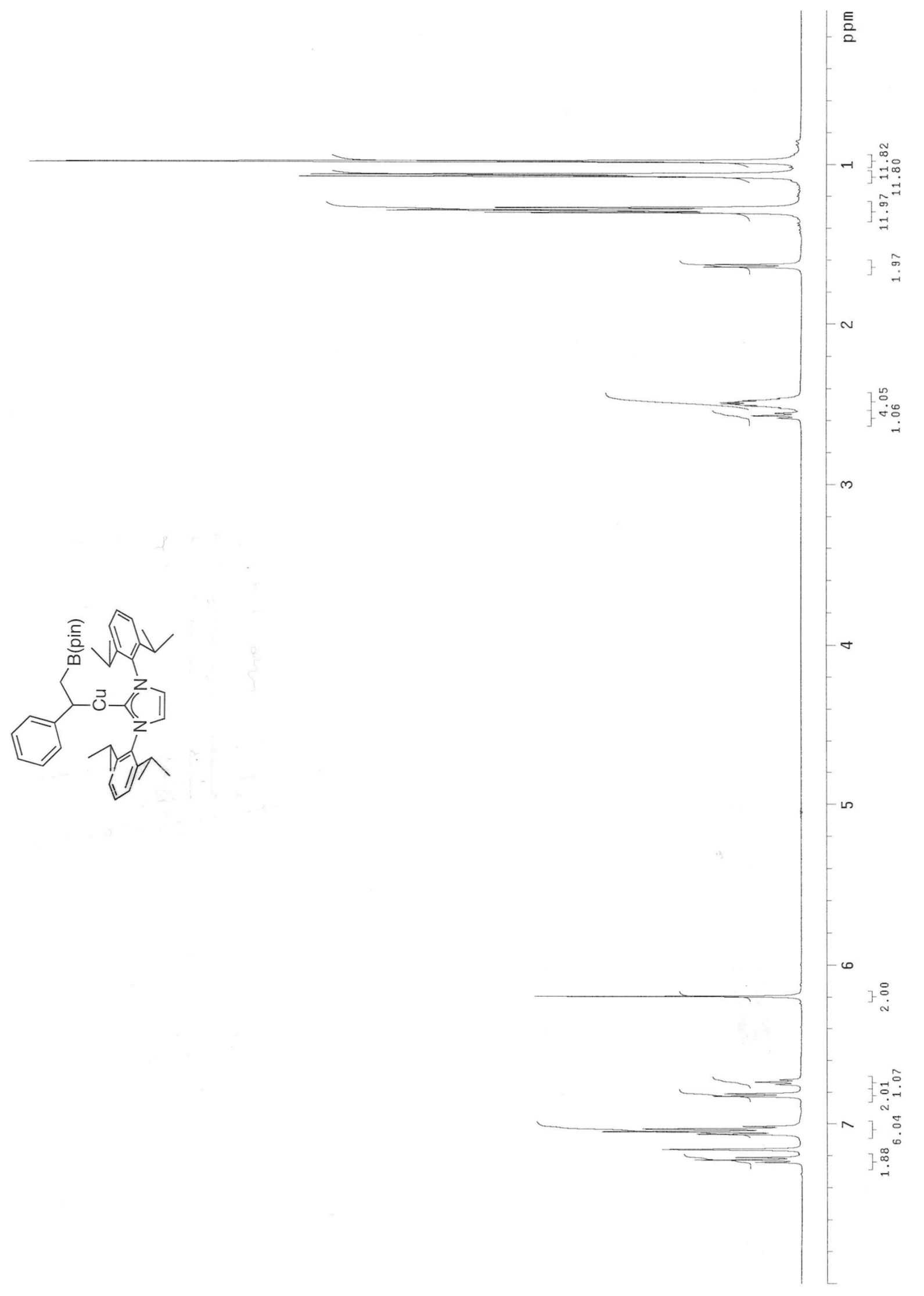




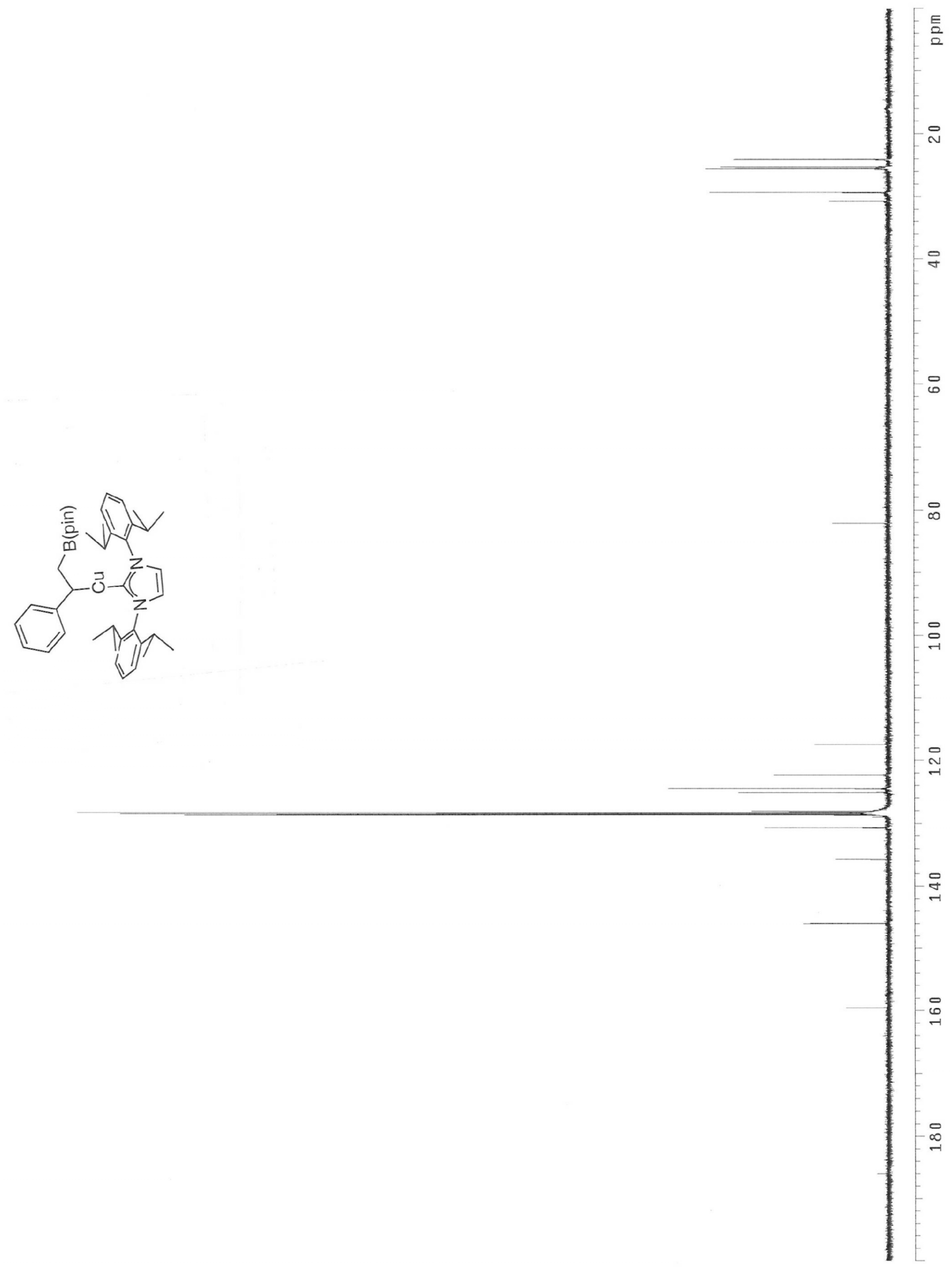




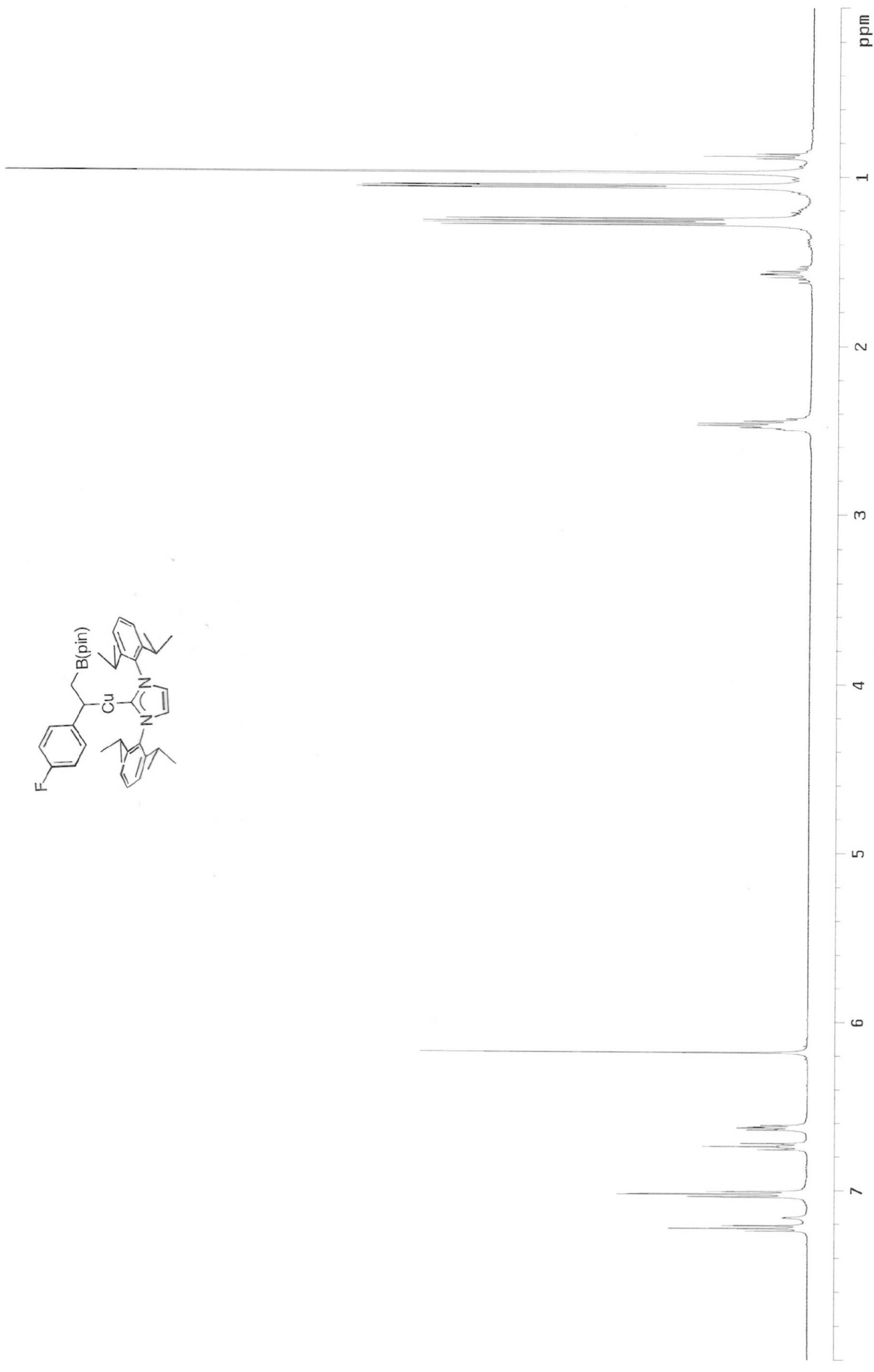

S12 

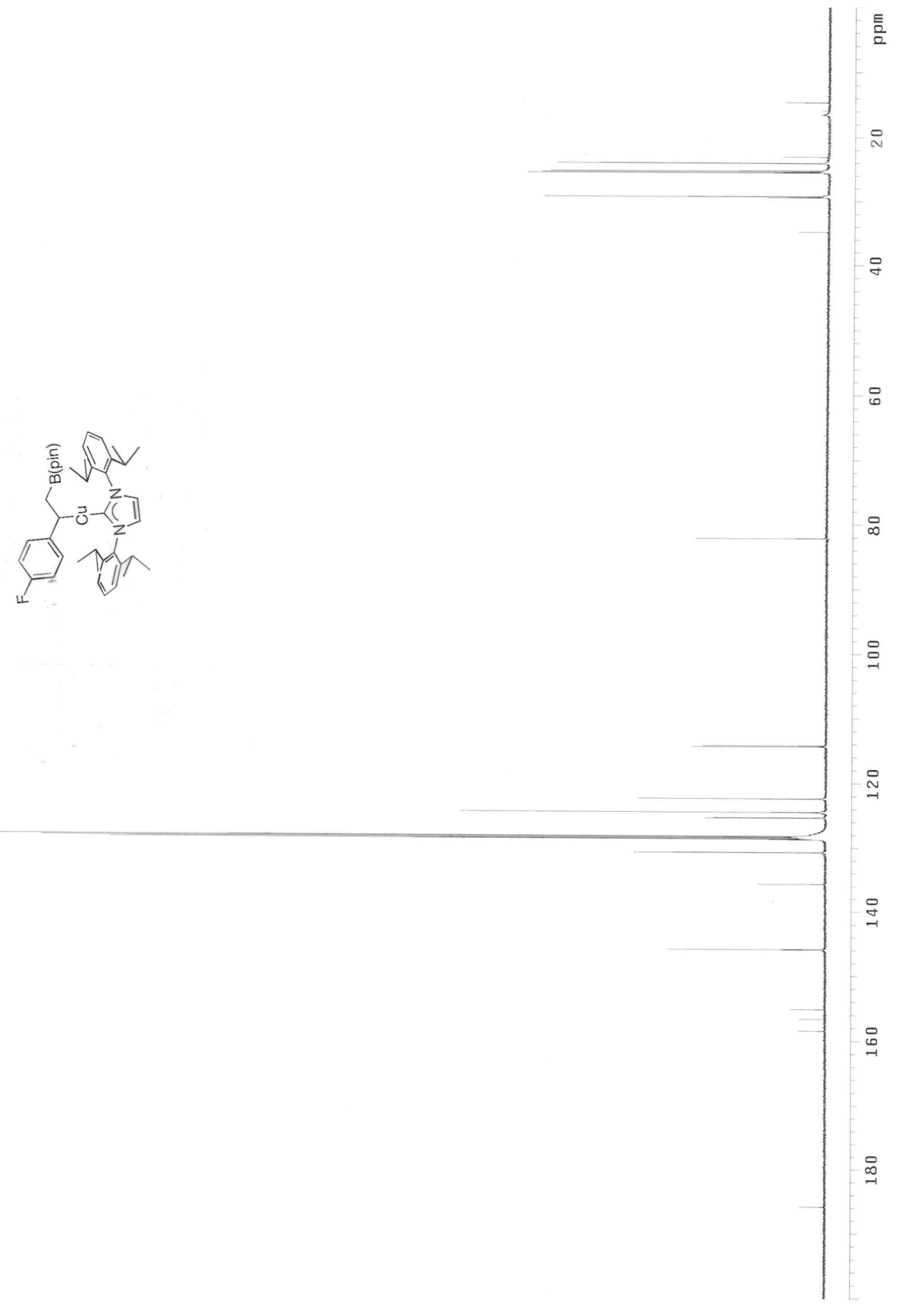


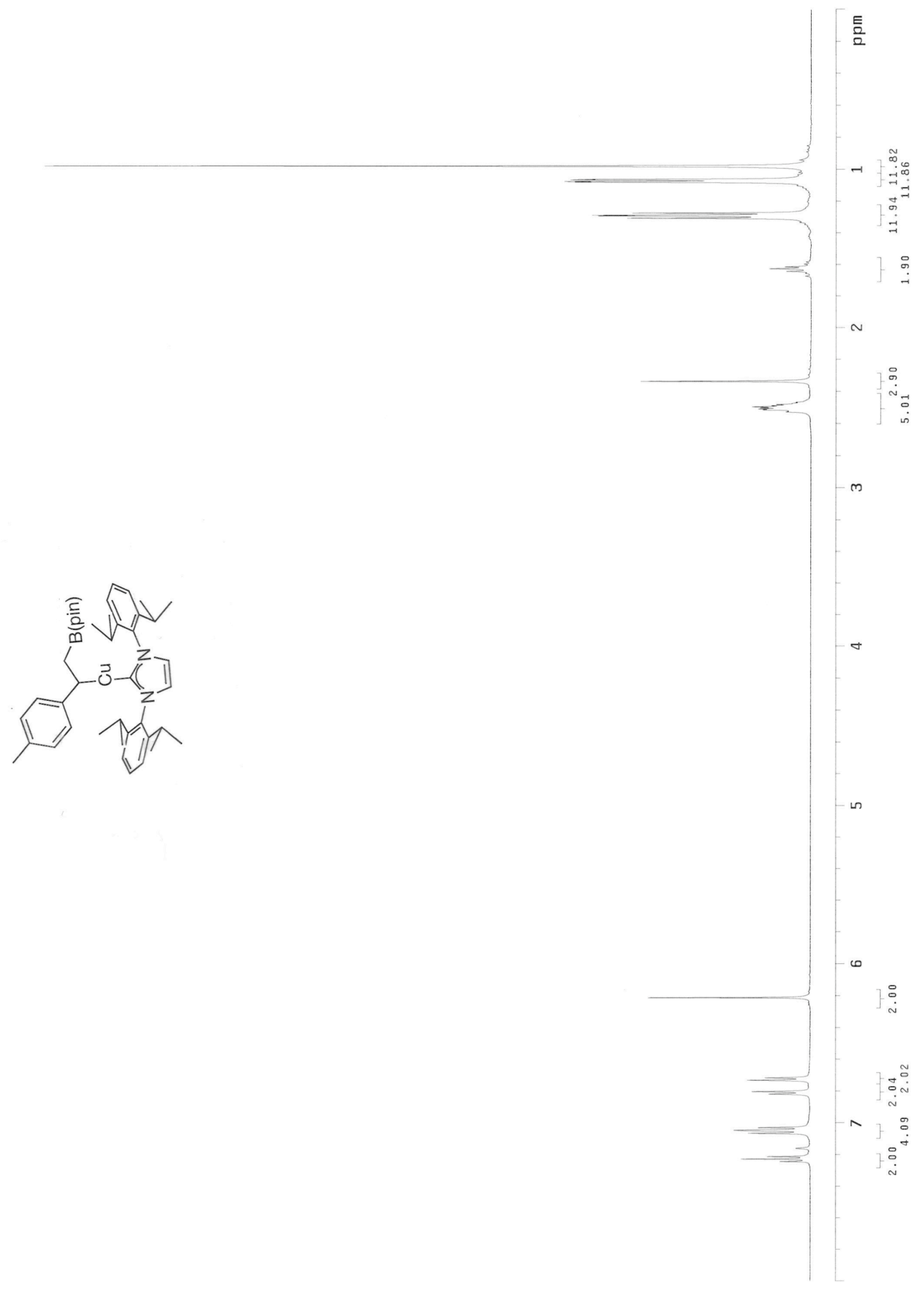




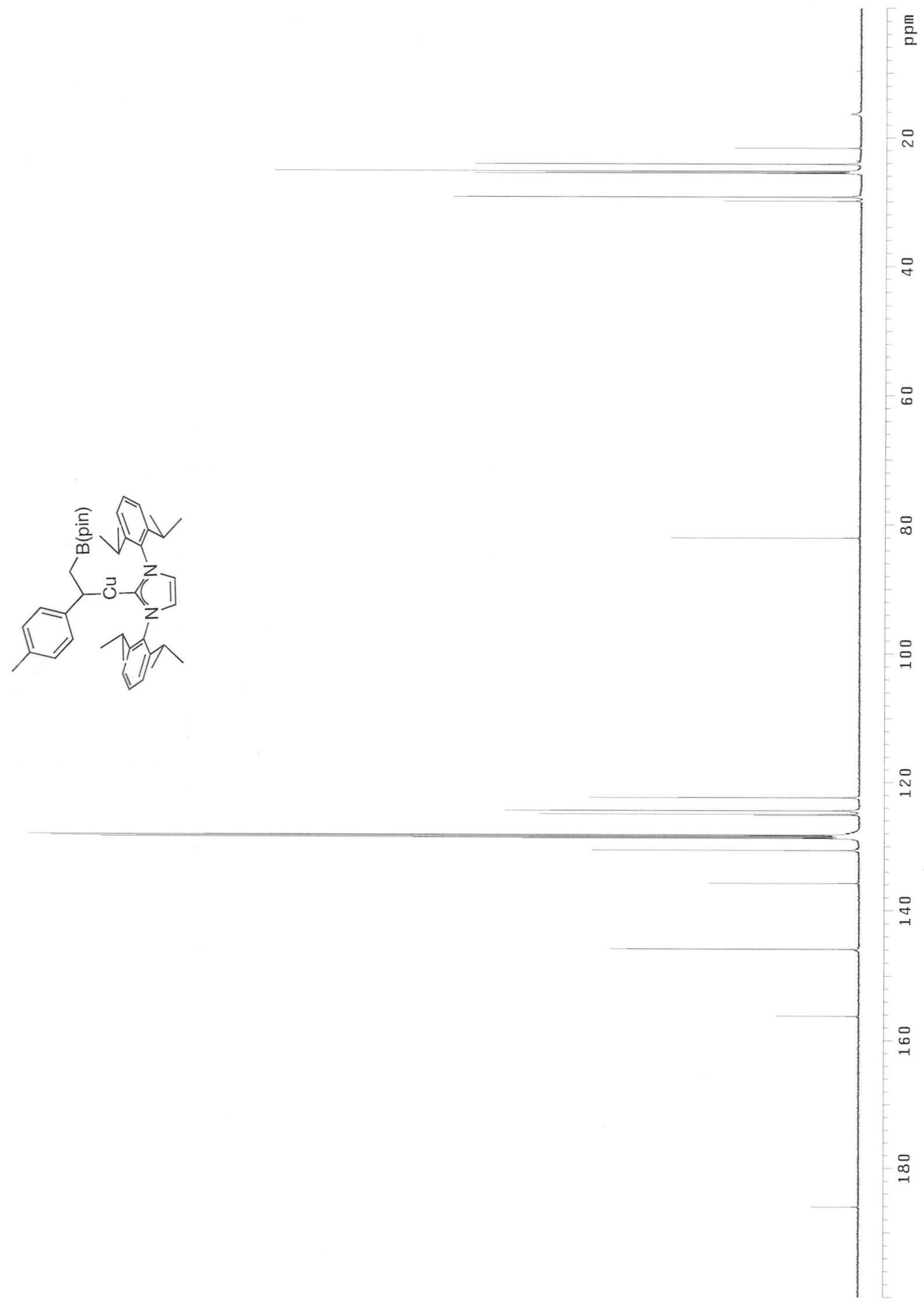




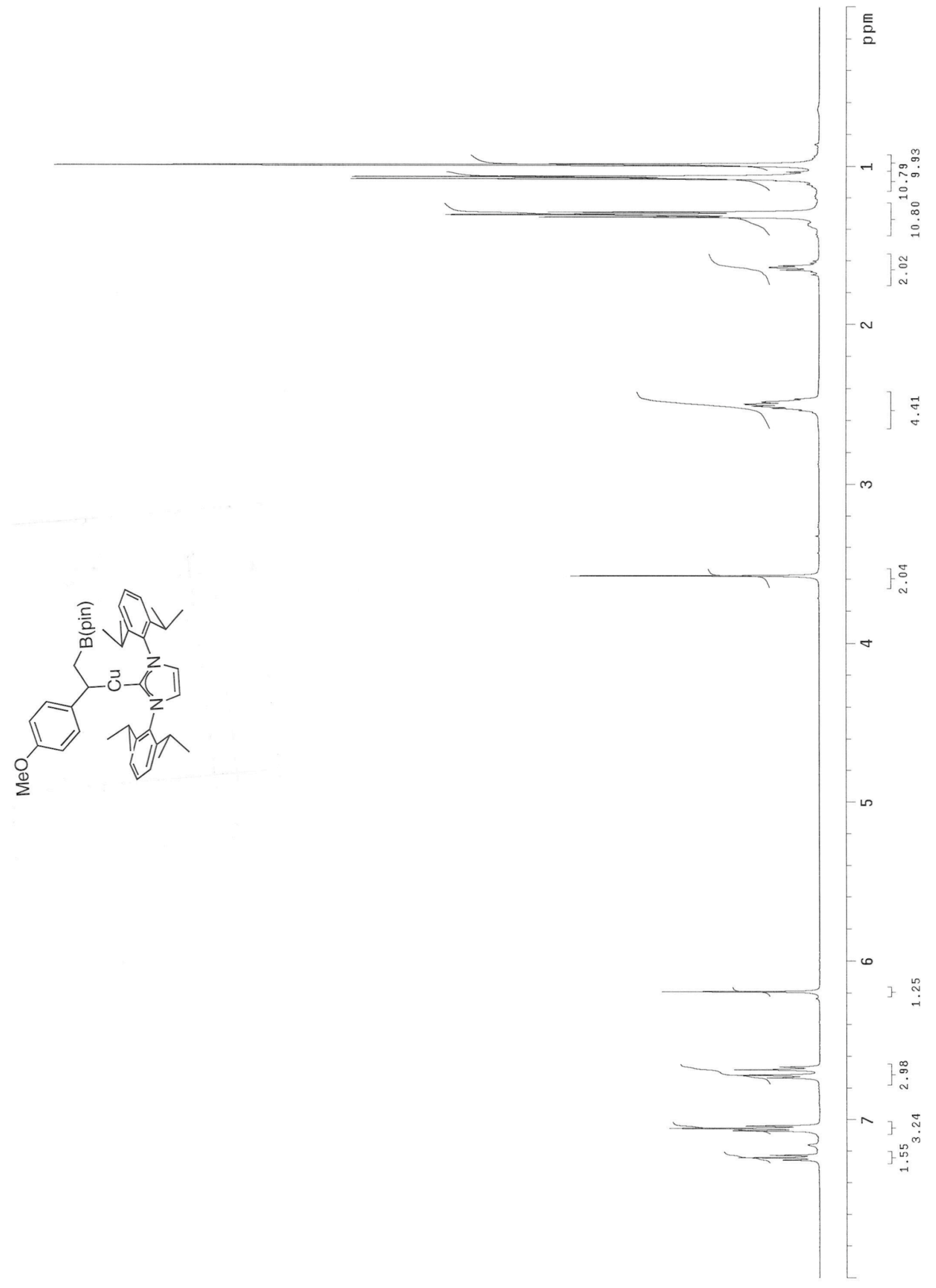




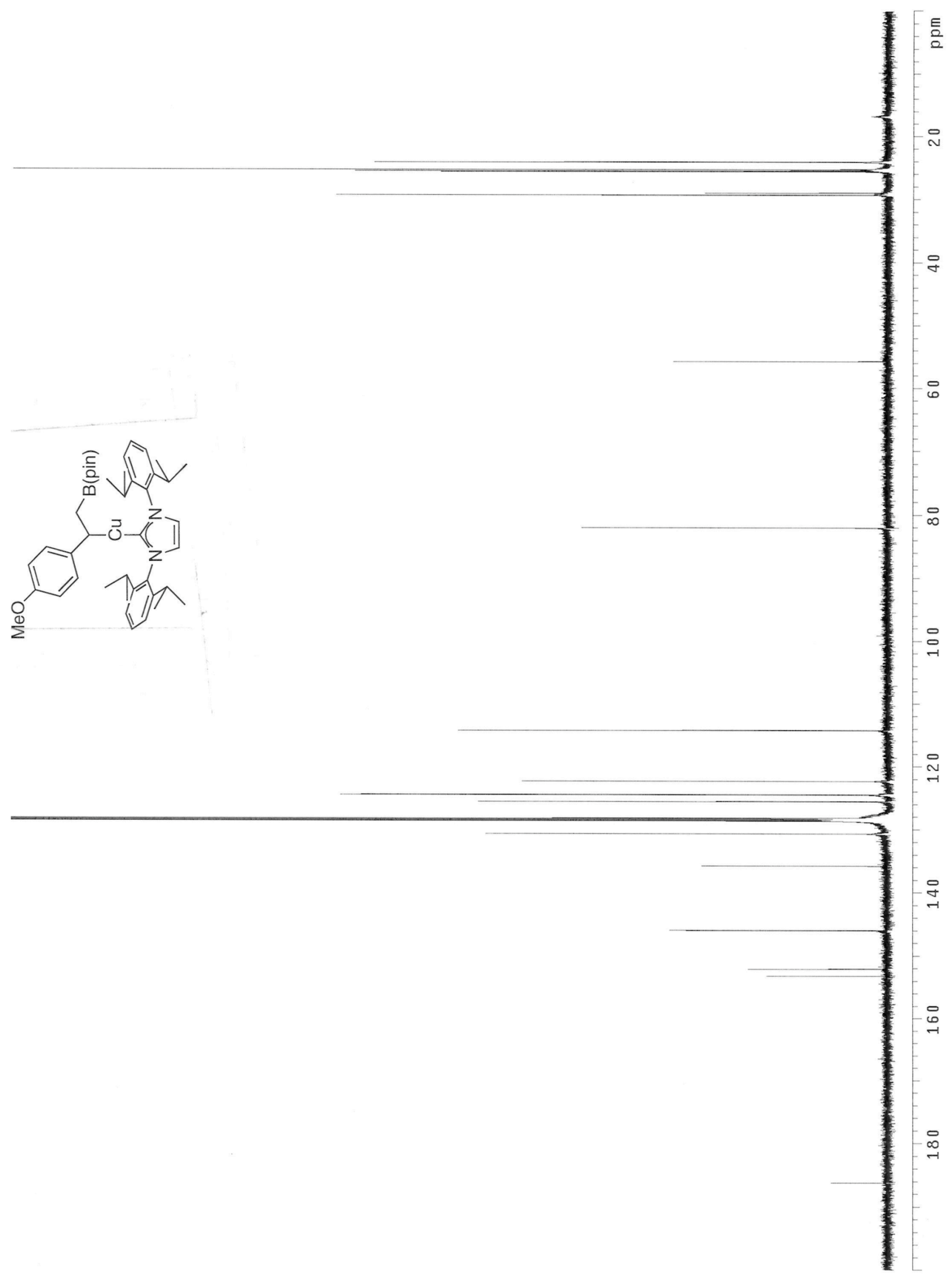




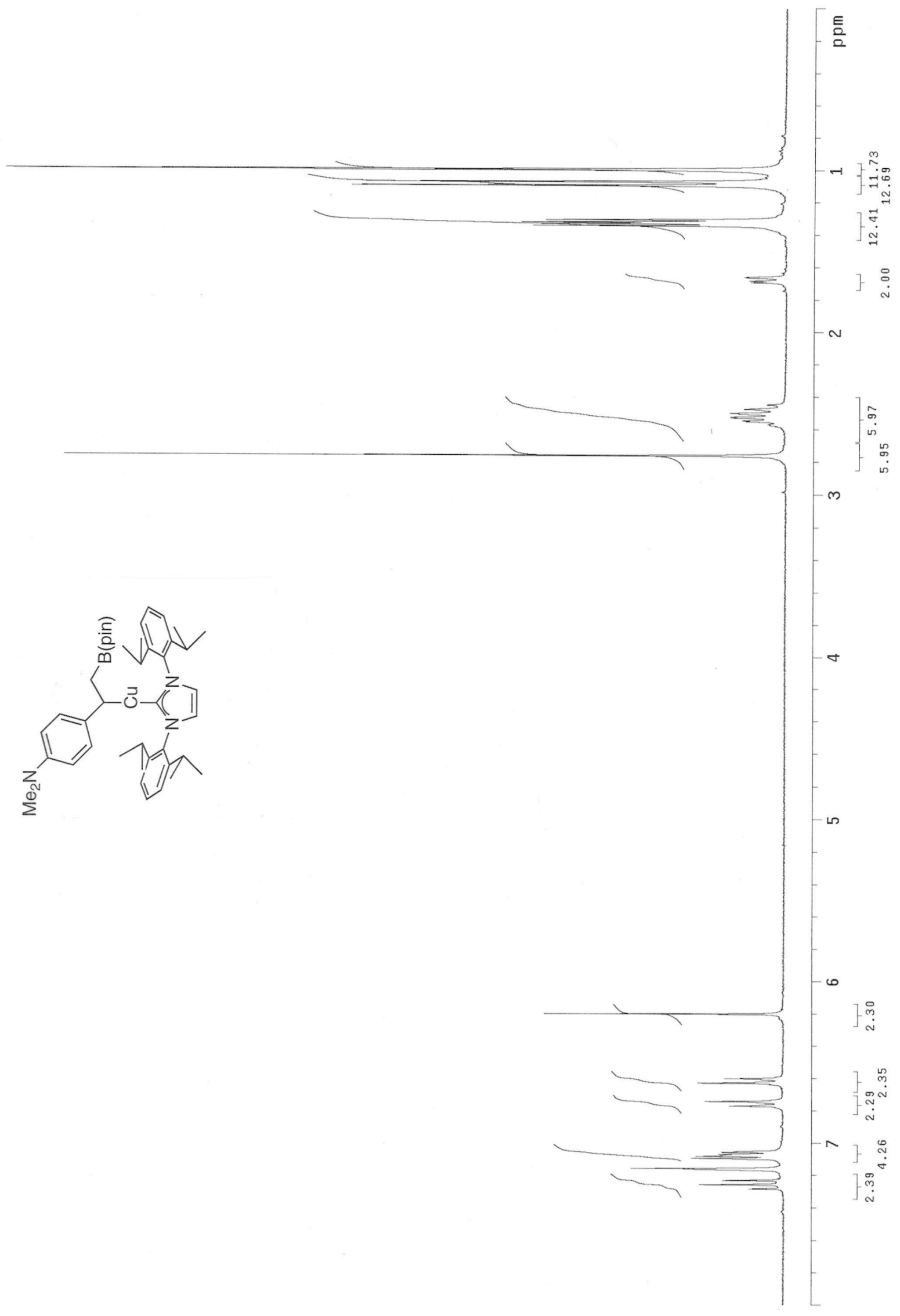




$$
4
$$




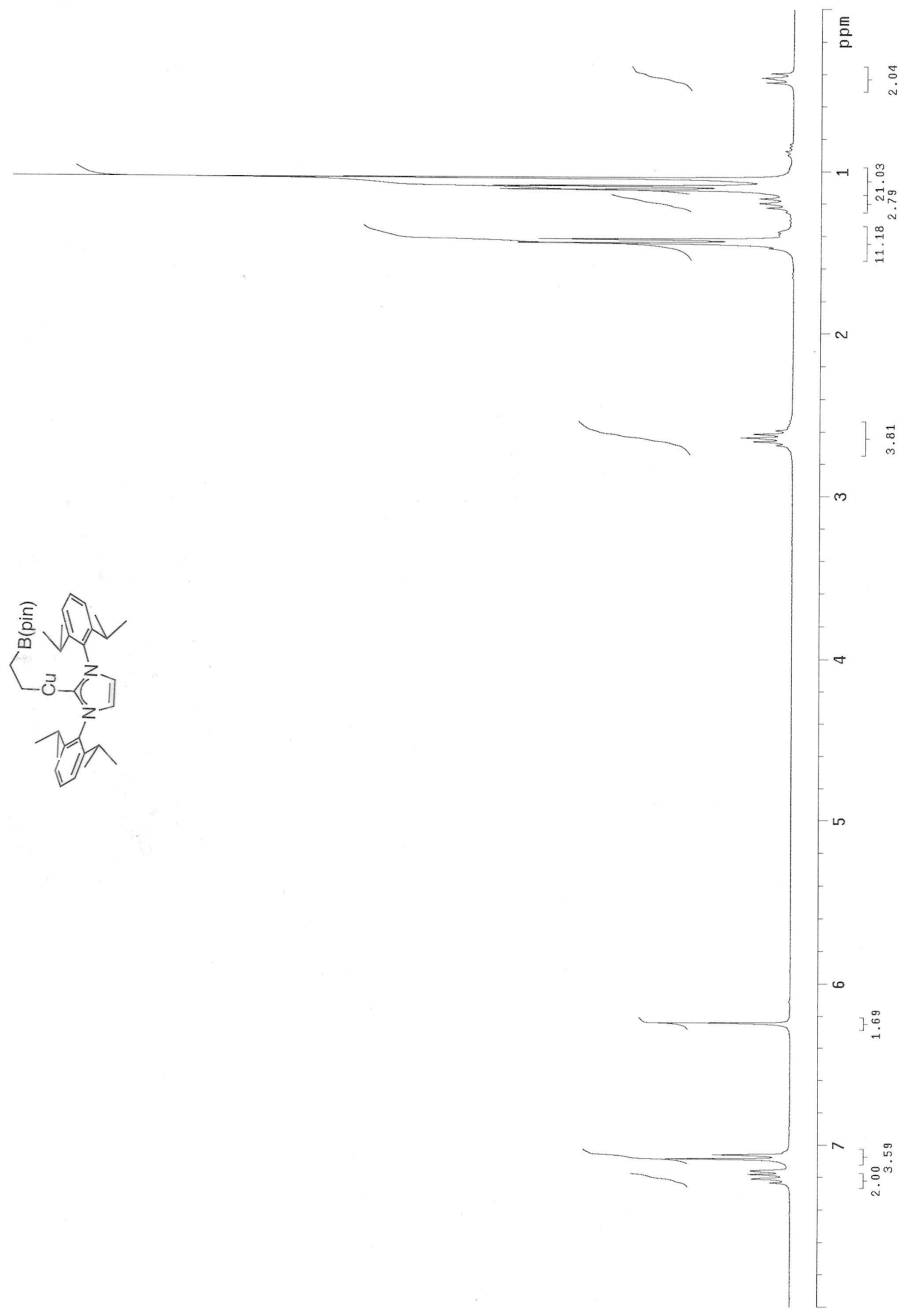




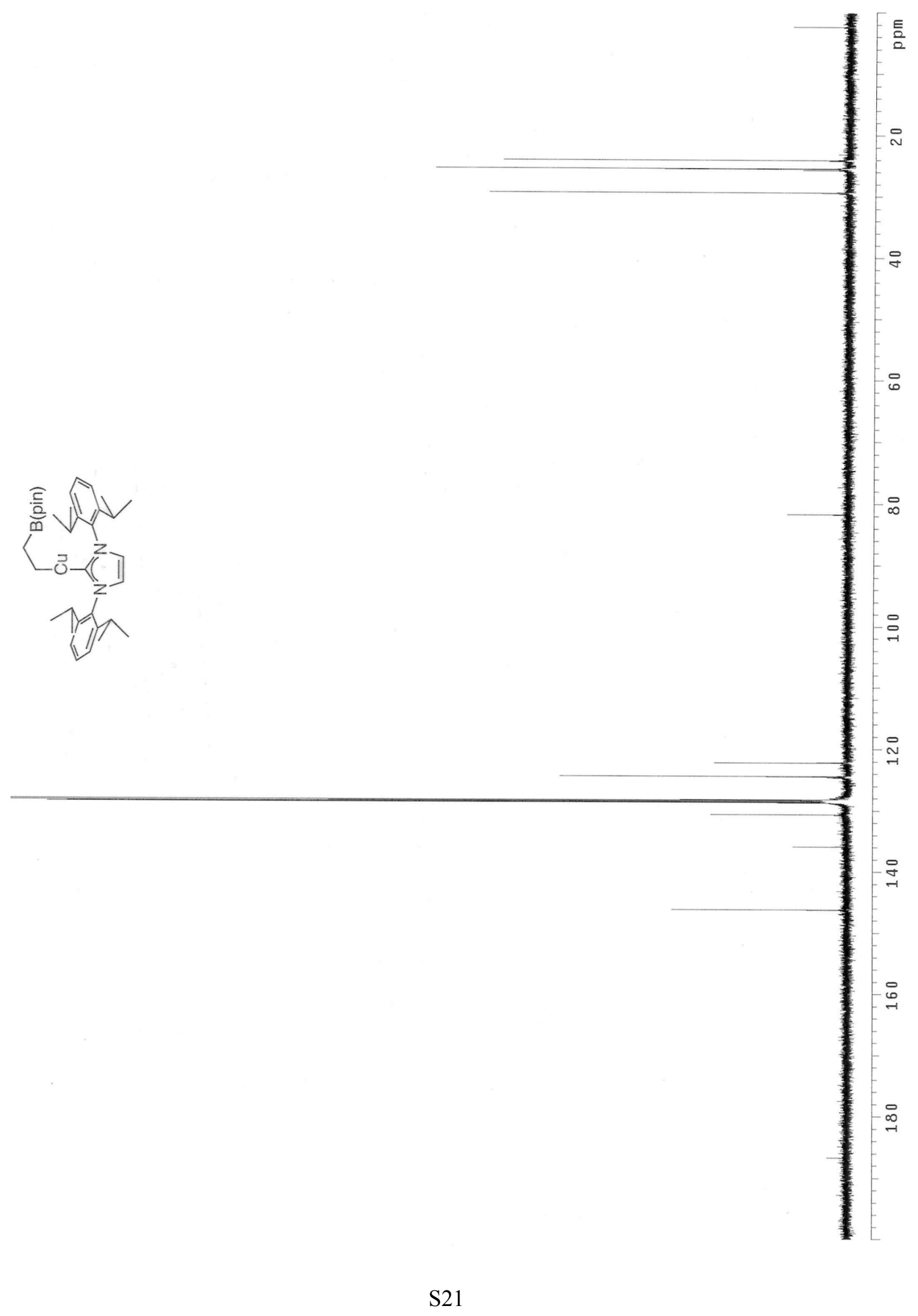




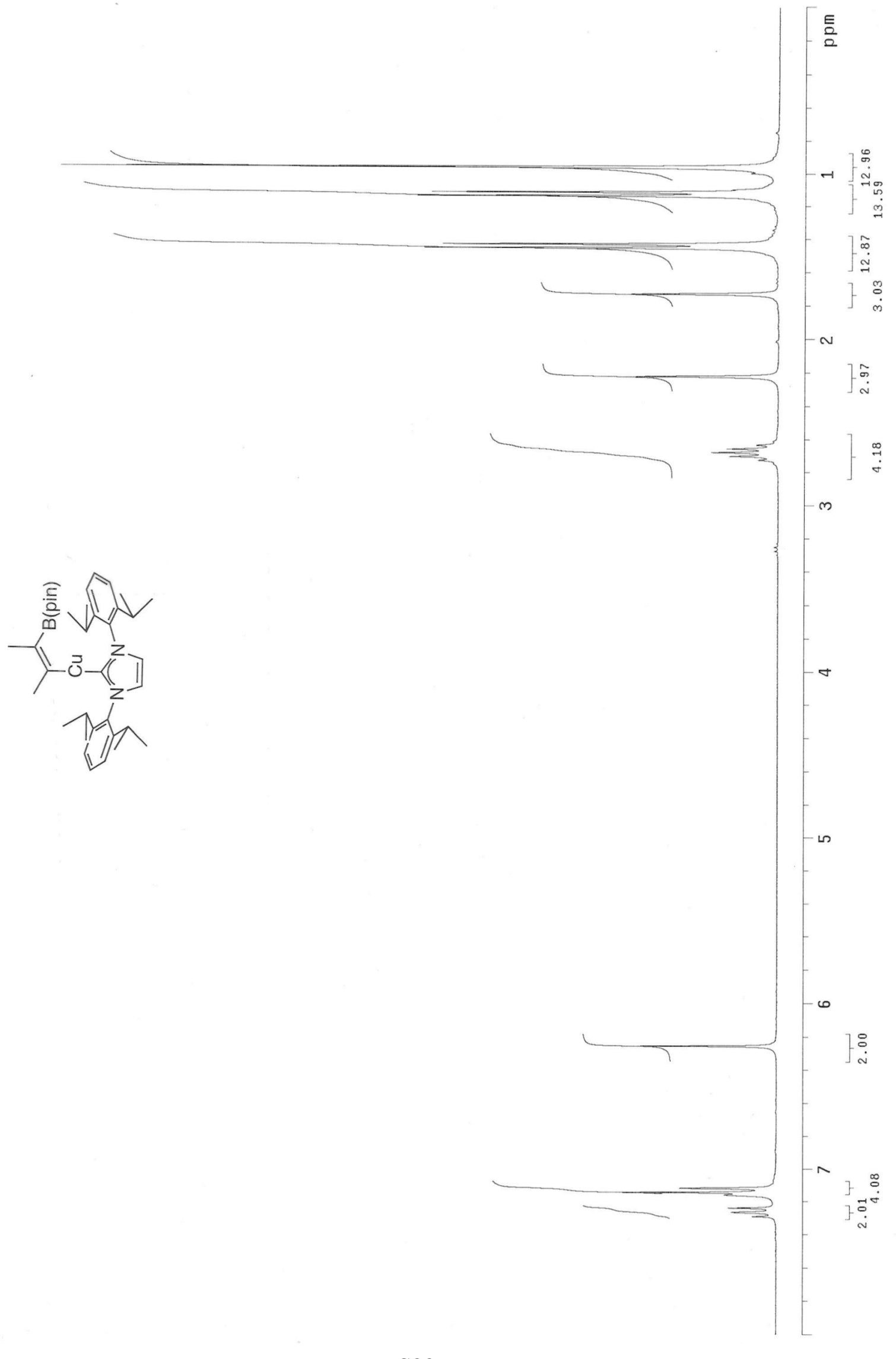




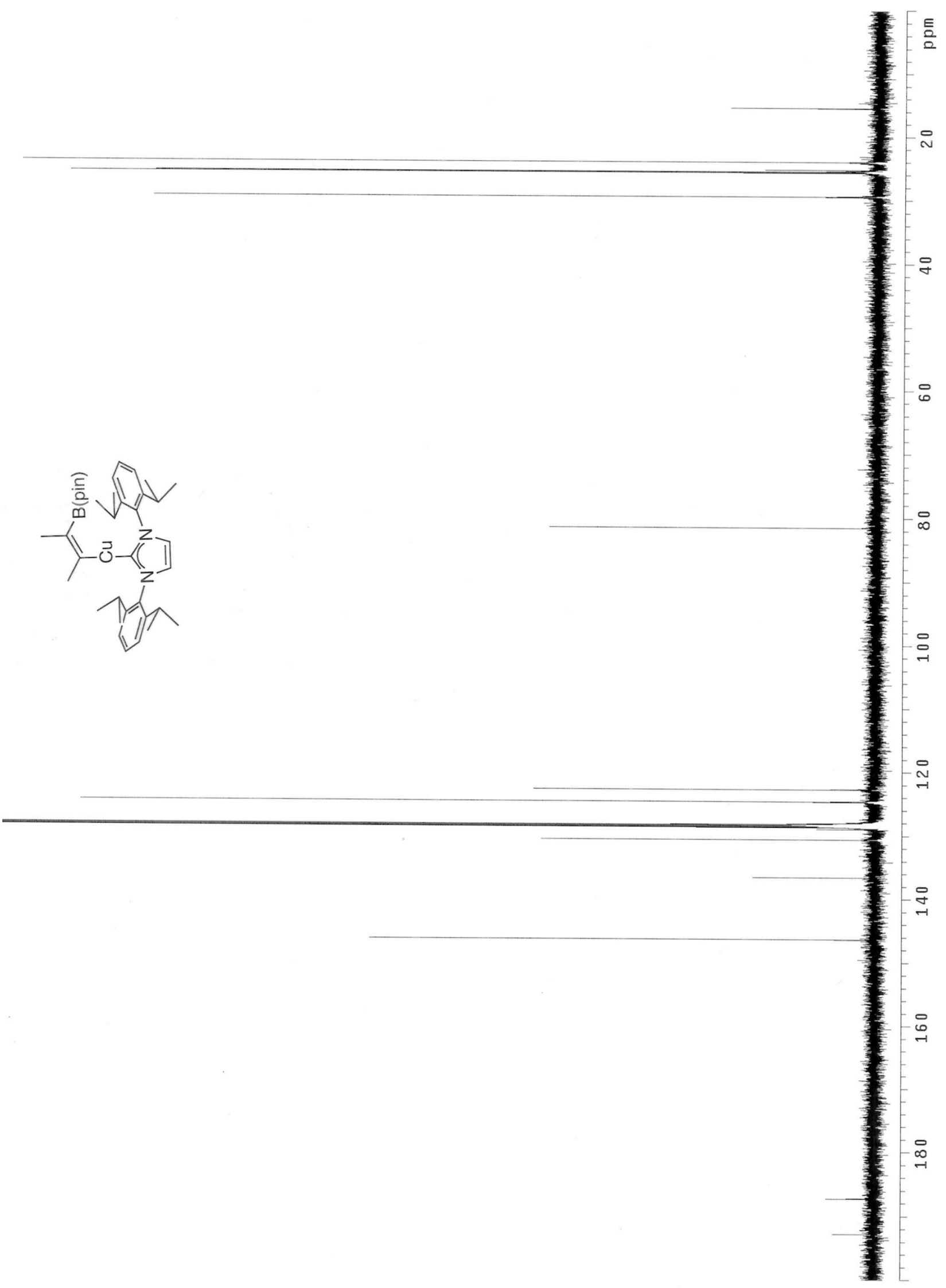

S23 


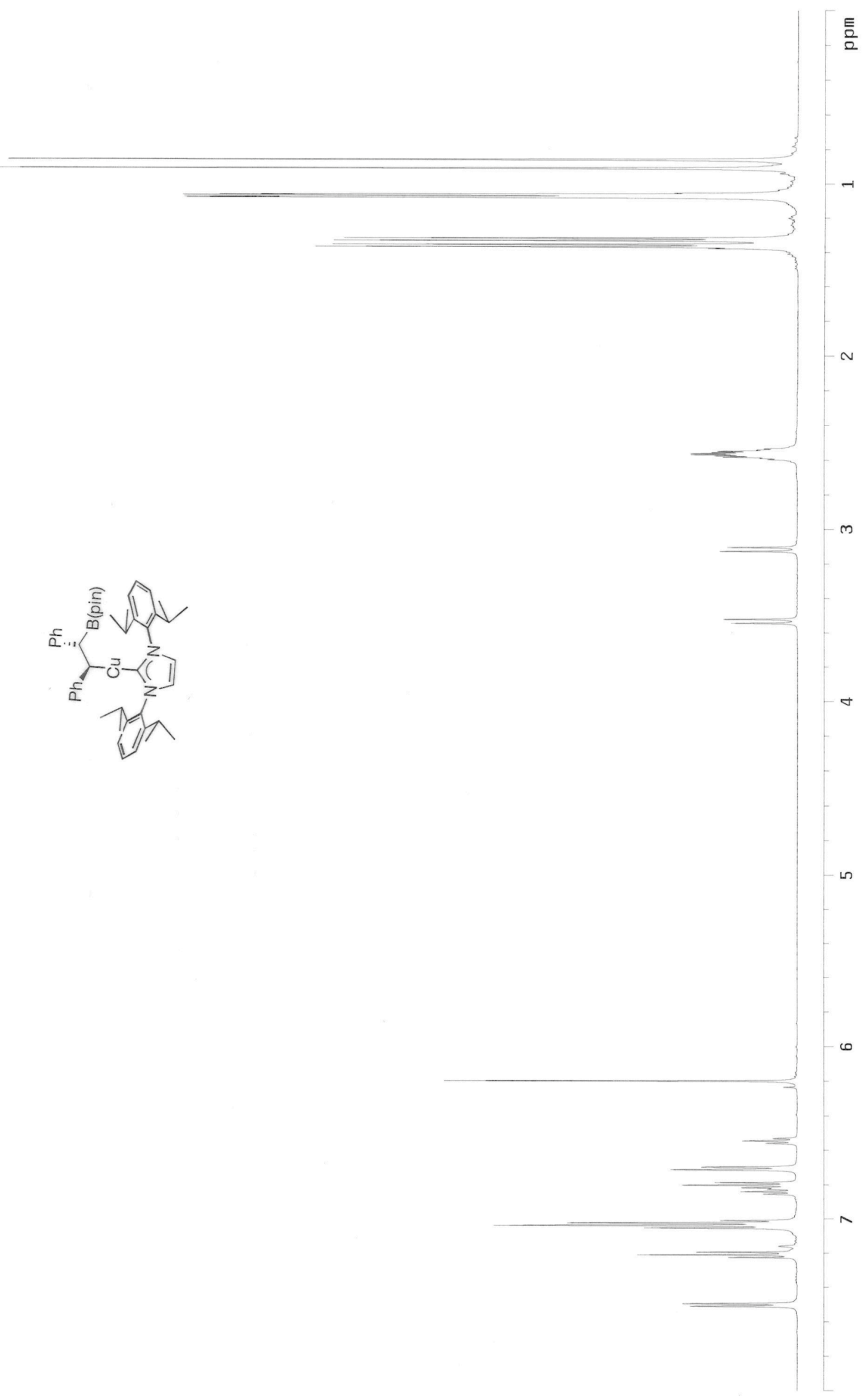




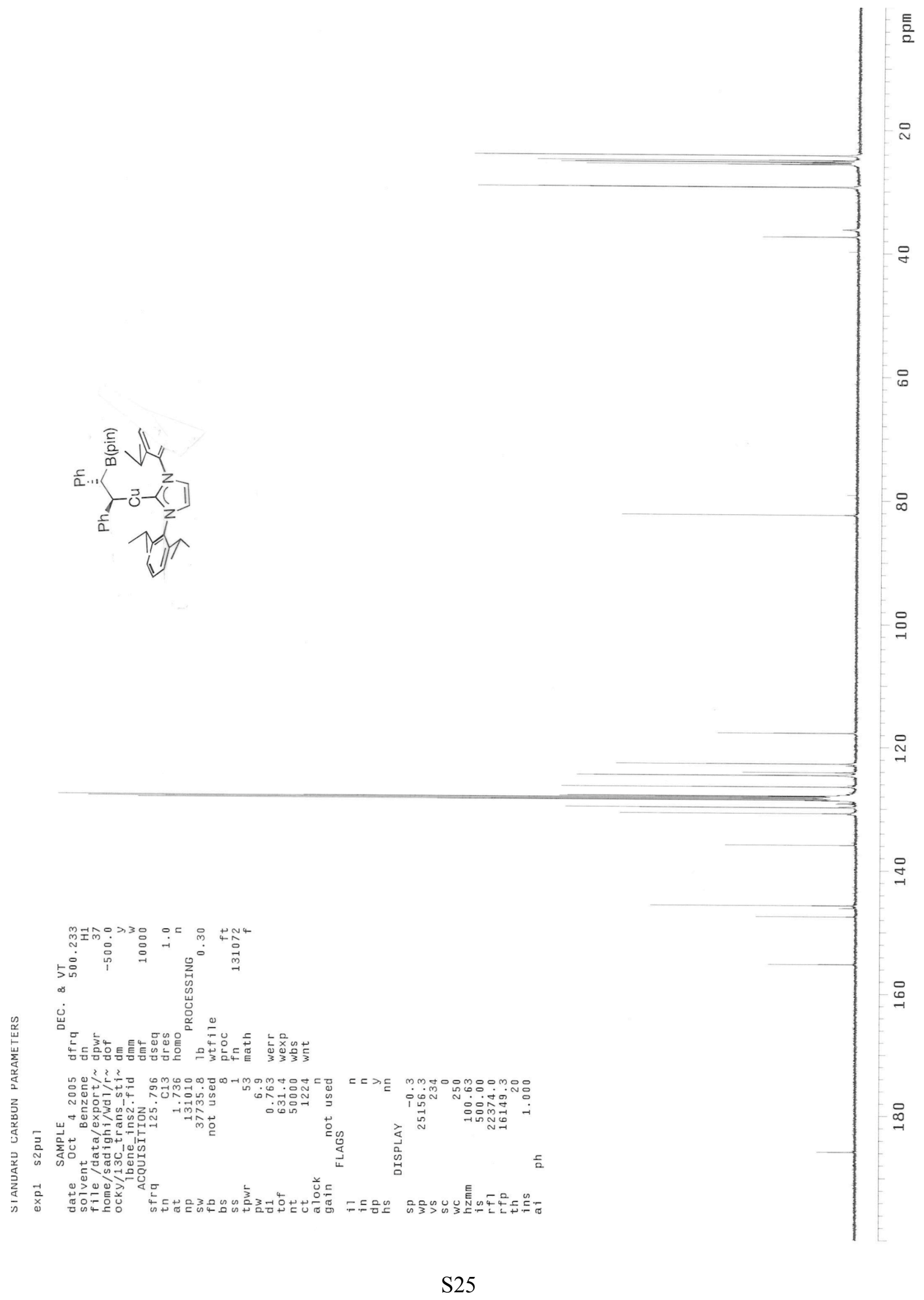




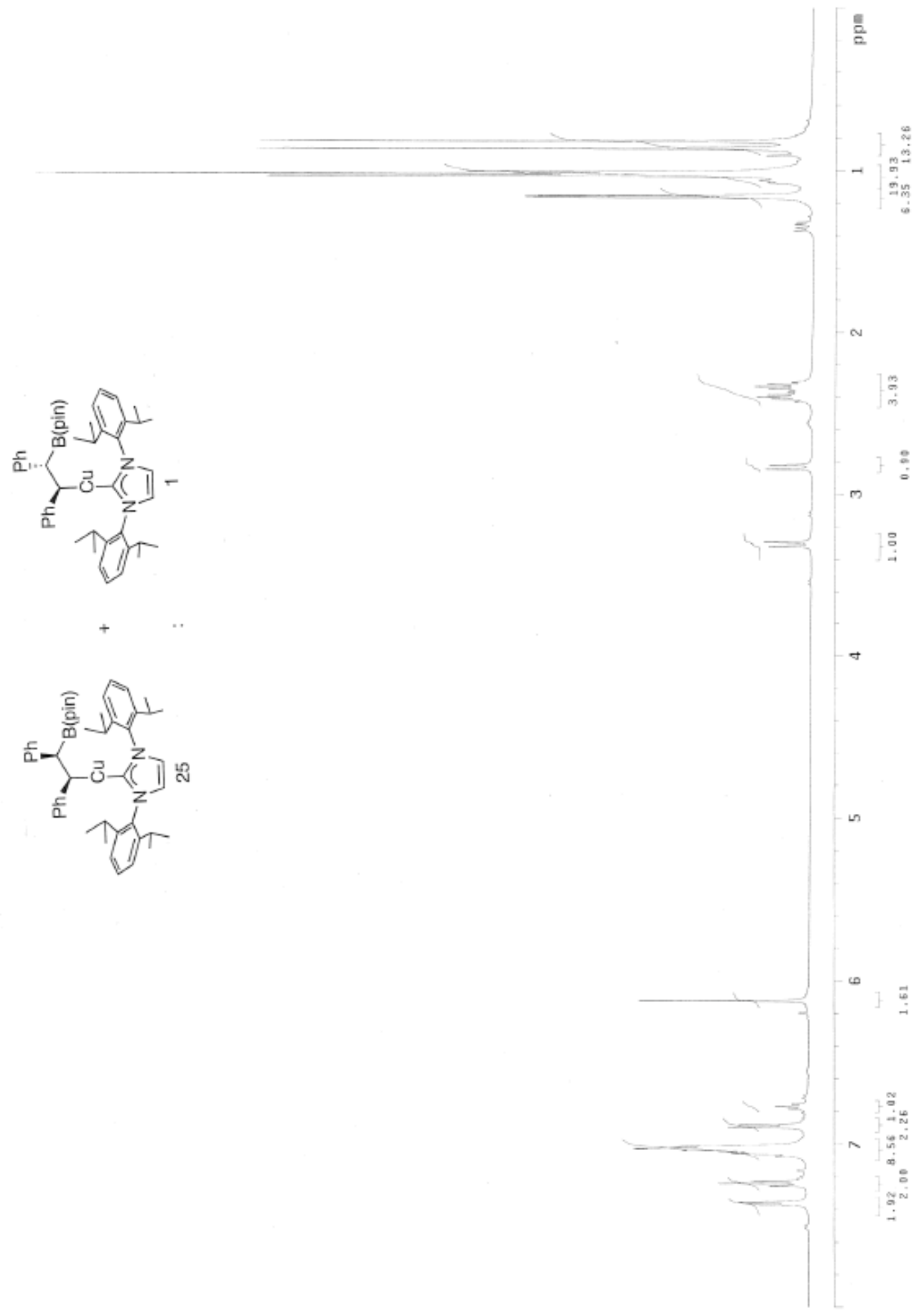




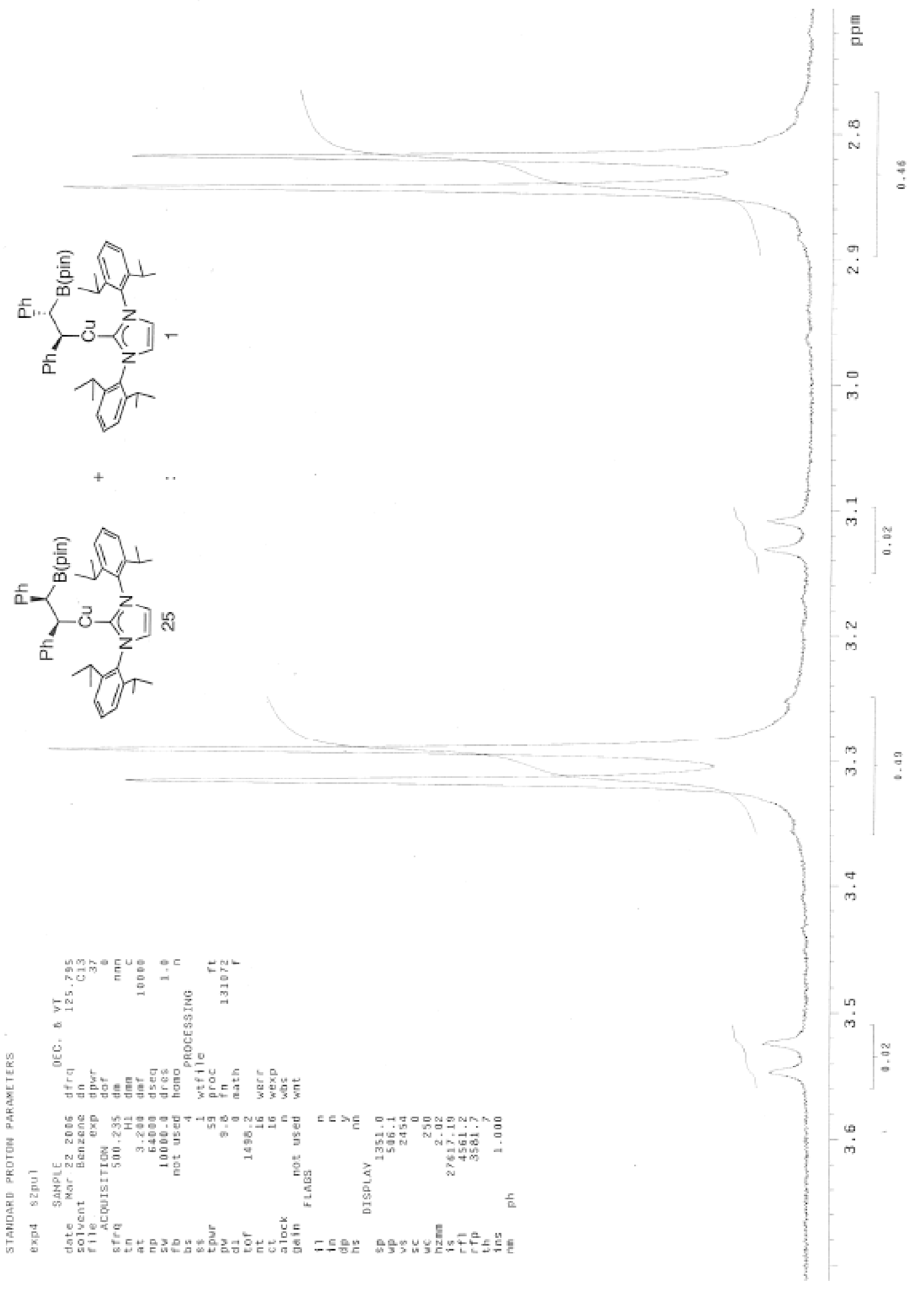




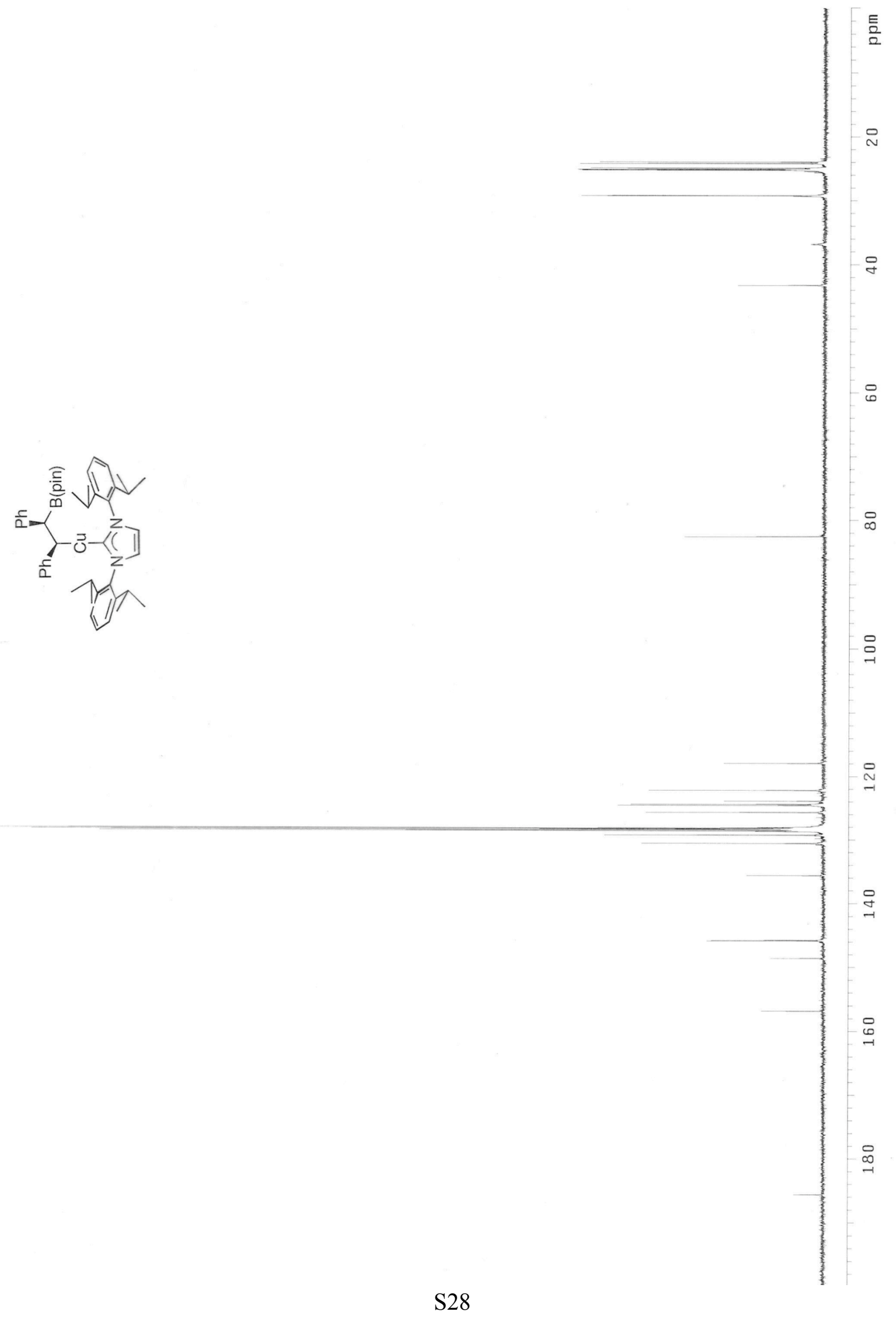




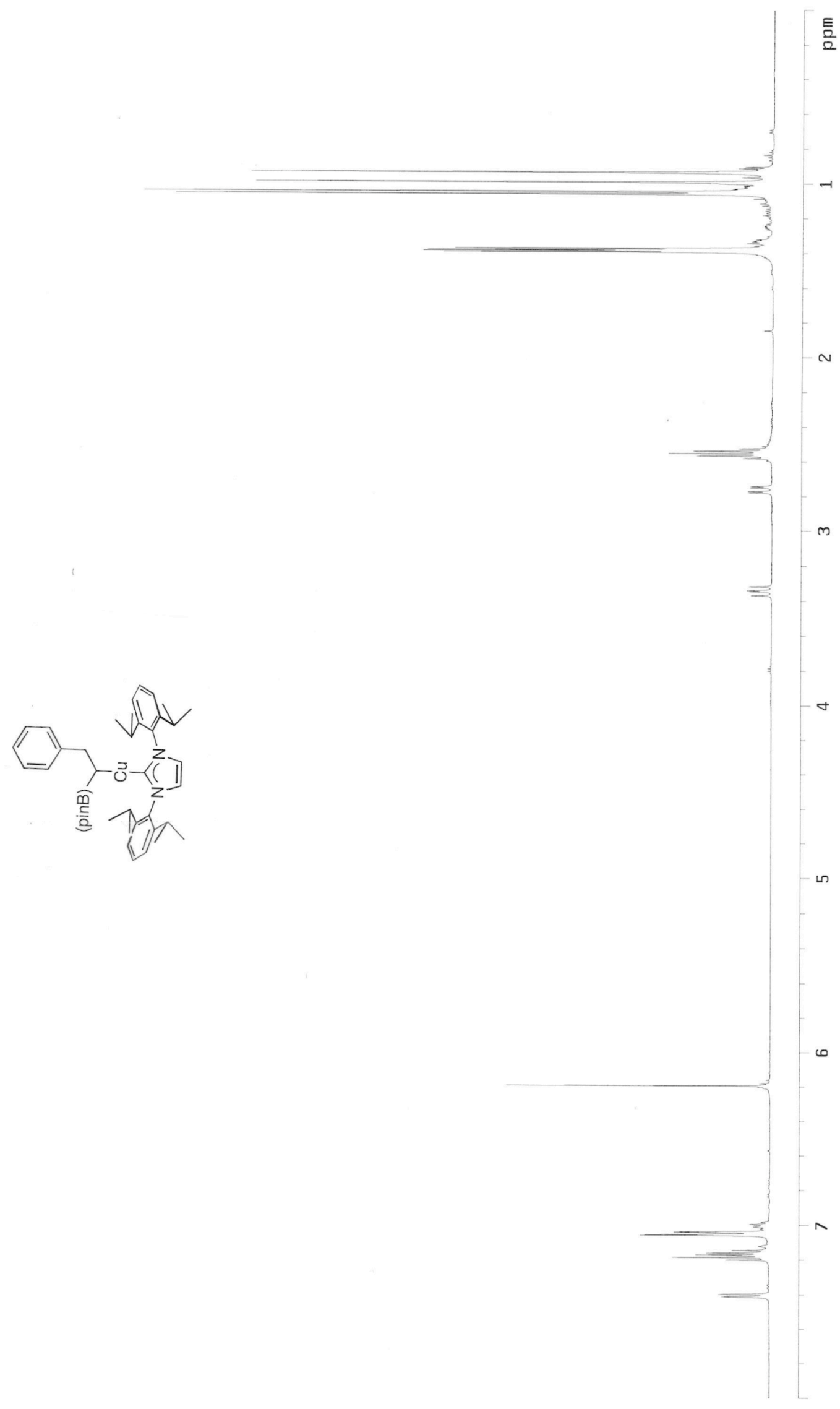




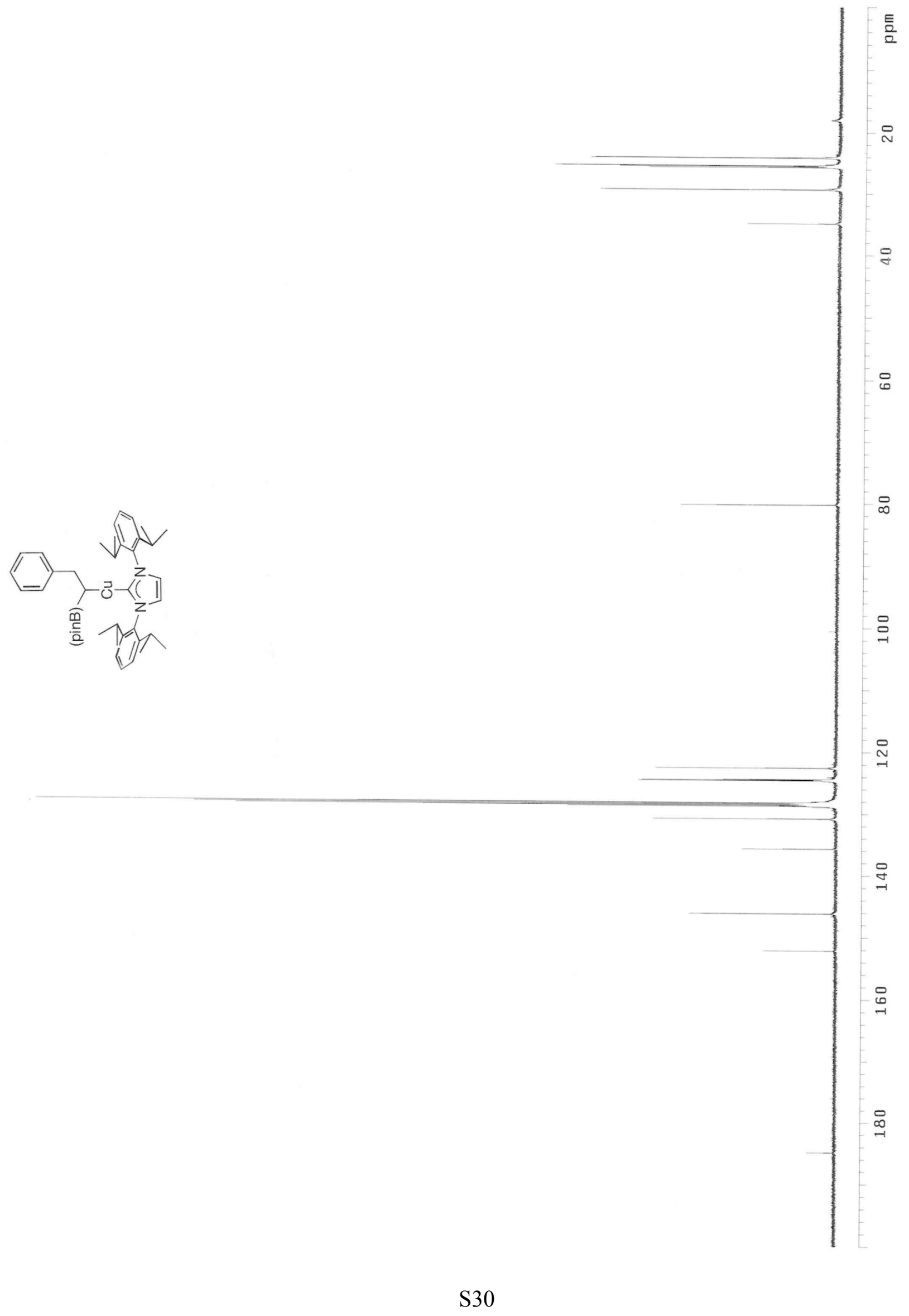


X-ray Diffraction Studies: Experiments were performed on single crystals of $\mathbf{1}$ and $\mathbf{2}$ grown by the vapor diffusion of pentanes into a concentrated ether solution (1) or pentanes into a toluene solution (2) at $-40{ }^{\circ} \mathrm{C}$. Colorless crystals were removed from the supernatant and transferred onto a microscope slide coated with Paratone $\mathrm{N}$ oil. Crystals were affixed to a glass fiber or a cryoloop using the oil, frozen in a nitrogen stream, and optically centered. The data were collected on a Siemens three-circle platform goniometer equipped with a Bruker Smart Apex CCD detector with graphite-monochromated Mo $\mathrm{K} \alpha$ radiation $(\lambda=0.71073 \AA)$, using both phi and omega scans at $-173{ }^{\circ} \mathrm{C}$. The structures were solved by direct methods (SHELXS) ${ }^{3}$ and refined against $F^{2}$ on all data by full matrix least squares with SHELXL-97 (Sheldrick, G. M. SHELXL 97; Universität Göttingen: Göttingen, Germany, 1997). All non-hydrogen atoms were refined anisotropically. Hydrogen atoms were placed at idealized positions and refined using a riding model.

\section{[1,3-Bis(2,6-diisopropylphenyl)imidazol-2-ylidene]copper(I) (1-phenyl)-2-pinacolatoboroethyl}

(1). An isopropyl group was disordered (C25, C27 and C28) and refined over two positions with the help of similarity restraints on 1-2 and 1-3 distances and displacement parameters. Rigid bond restraints for anisotropic displacement parameters were also used. A pentane molecule located on a special position was also found to be disordered and was refined with the aid of part -1 and part -2 instructions, as well as the restraints mentioned above. The relative occupancies for the disordered parts were refined freely, while constraining the overall occupation to unity. 


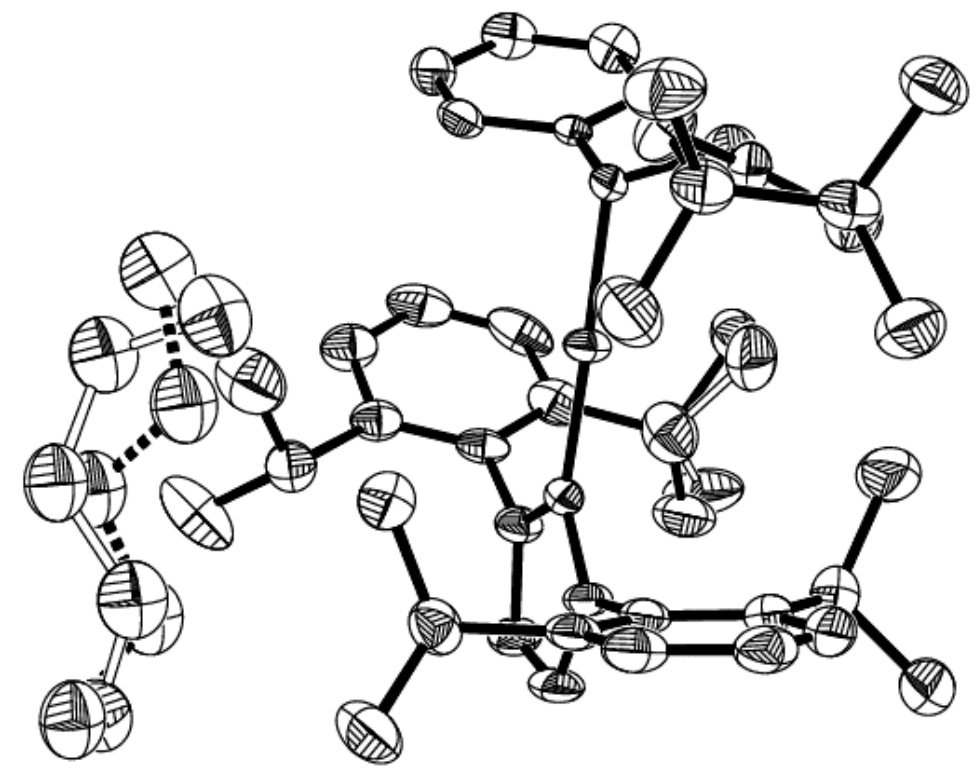

Figure S1. X-ray crystal structure of $\mathbf{1 \bullet 0 . 5}\left(\mathbf{C}_{\mathbf{5}} \mathbf{H}_{\mathbf{1 2}}\right)$ shown as $50 \%$ ellipsoids. For clarity, hydrogen atoms have been omitted.

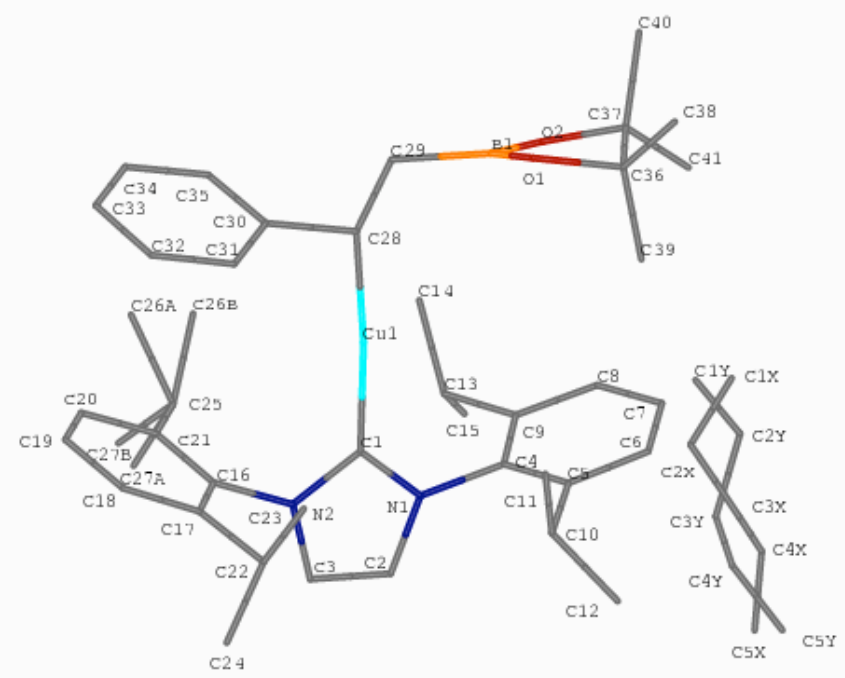

Figure S2. X-ray crystal structure of $\mathbf{1 \bullet 0 . 5}\left(\mathbf{C}_{\mathbf{5}} \mathbf{H}_{\mathbf{1 2}}\right)$ shown as a stick figure for clarity in labeling.

Table S1. Crystal data and structure refinement for $\mathbf{1 \bullet 0 . 5}\left(\mathbf{C}_{5} \mathbf{H}_{12}\right)$.

Identification code

Empirical formula

Formula weight

Temperature

Wavelength
05141

$\mathrm{C}_{43.50} \mathrm{H}_{62} \mathrm{BCuN}_{2} \mathrm{O}_{2}$

719.30

100(2) K

$0.71073 \AA$ 
Crystal system

Space group

Unit cell dimensions

Volume

Z

Density (calculated)

Absorption coefficient

$\mathrm{F}(000)$

Crystal size

Theta range for data collection

Index ranges

Reflections collected

Independent reflections

Completeness to theta $=25.02^{\circ}$

Absorption correction

Max. and min. transmission

Refinement method

Data / restraints / parameters

Goodness-of-fit on $\mathrm{F}^{2}$

Final $\mathrm{R}$ indices $[\mathrm{I}>2 \sigma(\mathrm{I})]$

$\mathrm{R}$ indices (all data)

Largest diff. peak and hole
Monoclinic

$\mathrm{P} 2(1) / \mathrm{n}$

$$
\begin{array}{ll}
\mathrm{a}=20.3264(8) \AA & \alpha=90^{\circ} . \\
\mathrm{b}=9.6981(4) \AA & \beta=106.996(2)^{\circ} . \\
\mathrm{c}=21.9595(8) \AA & \gamma=90^{\circ} .
\end{array}
$$

4139.8(3) $\AA^{3}$

4

$1.154 \mathrm{Mg} / \mathrm{m}^{3}$

$0.563 \mathrm{~mm}^{-1}$

1548

$0.20 \times 0.05 \times 0.05 \mathrm{~mm}^{3}$

1.94 to $25.02^{\circ}$.

$-23<=\mathrm{h}<=24,-11<=\mathrm{k}<=11,-26<=\mathrm{l}<=25$

46339

$7312[\mathrm{R}(\mathrm{int})=0.0826]$

$100.0 \%$

Semi-empirical from equivalents

0.9724 and 0.8957

Full-matrix least-squares on $\mathrm{F}^{2}$

7312 / 285 / 544

1.133

$\mathrm{R} 1=0.0657, \mathrm{wR} 2=0.1349$

$\mathrm{R} 1=0.0929, \mathrm{wR} 2=0.1449$

0.625 and $-0.365 \mathrm{e} / \AA^{3}$

Table 2. Atomic coordinates $\left(\times 10^{4}\right)$ and equivalent isotropic displacement parameters $\left(\AA^{2} \times 10^{3}\right)$ for $\mathbf{1 \bullet 0 . 5}\left(\mathbf{C}_{5} \mathbf{H}_{12}\right)$. U(eq) is defined as one third of the trace of the orthogonalized $\mathrm{U}^{\mathrm{ij}}$ tensor.

\begin{tabular}{lrrrr}
\hline & $\mathrm{x}$ & $\mathrm{y}$ & $\mathrm{z}$ & $\mathrm{U}(\mathrm{eq})$ \\
\hline $\mathrm{Cu}(1)$ & $8944(1)$ & $4767(1)$ & $1426(1)$ & $26(1)$ \\
$\mathrm{C}(28)$ & $9018(2)$ & $6765(3)$ & $1380(2)$ & $23(1)$ \\
$\mathrm{N}(2)$ & $8203(2)$ & $2175(3)$ & $1105(2)$ & $30(1)$ \\
$\mathrm{N}(1)$ & $9228(2)$ & $1784(3)$ & $1687(2)$ & $29(1)$ \\
$\mathrm{C}(1)$ & $8799(2)$ & $2833(4)$ & $1413(2)$ & $26(1)$ \\
$\mathrm{C}(30)$ & $8350(2)$ & $7206(3)$ & $923(2)$ & $23(1)$ \\
$\mathrm{C}(31)$ & $7742(2)$ & $7279(4)$ & $1106(2)$ & $31(1)$
\end{tabular}




\begin{tabular}{|c|c|c|c|c|}
\hline $\mathrm{O}(2)$ & $10946(2)$ & $6823(3)$ & $1854(2)$ & $45(1)$ \\
\hline $\mathrm{O}(1)$ & $10283(2)$ & $7796(3)$ & $2409(1)$ & $45(1)$ \\
\hline$C(17)$ & $7101(2)$ & $3204(4)$ & $1051(2)$ & $34(1)$ \\
\hline $\mathrm{C}(29)$ & $9665(2)$ & $7279(4)$ & $1234(2)$ & $36(1)$ \\
\hline$C(22)$ & $7180(2)$ & $2838(5)$ & $1741(2)$ & $39(1)$ \\
\hline$C(35)$ & $8289(2)$ & $7571(4)$ & $295(2)$ & $31(1)$ \\
\hline$C(21)$ & $7535(2)$ & $3288(4)$ & $122(2)$ & $41(1)$ \\
\hline$C(25)$ & $8080(3)$ & $2928(5)$ & $-199(2)$ & $57(1)$ \\
\hline$C(26 A)$ & $8123(9)$ & $4105(8)$ & $-685(6)$ & $63(4)$ \\
\hline$C(27 A)$ & $7880(11)$ & $1598(13)$ & $-571(8)$ & $75(5)$ \\
\hline$C(26 B)$ & $8538(13)$ & $4030(17)$ & $-320(16)$ & $65(7)$ \\
\hline $\mathrm{C}(27 \mathrm{~B})$ & $7870(20)$ & $1870(30)$ & $-733(15)$ & $78(9)$ \\
\hline $\mathrm{B}(1)$ & $10312(2)$ & $7286(5)$ & $1844(2)$ & $34(1)$ \\
\hline$C(37)$ & $11404(2)$ & $7198(4)$ & $2466(2)$ & $35(1)$ \\
\hline $\mathrm{C}(2)$ & $8906(2)$ & $517(4)$ & $1551(2)$ & $38(1)$ \\
\hline $\mathrm{C}(9)$ & $10434(2)$ & $2042(4)$ & $1739(2)$ & $37(1)$ \\
\hline$C(7)$ & $11273(2)$ & $2361(4)$ & $2760(3)$ & $44(1)$ \\
\hline$C(6)$ & $10768(2)$ & $2296(4)$ & $3061(2)$ & $40(1)$ \\
\hline$C(32)$ & $7130(2)$ & $7711(4)$ & $692(2)$ & $38(1)$ \\
\hline$C(4)$ & $9934(2)$ & $1977(4)$ & $2056(2)$ & $31(1)$ \\
\hline$C(34)$ & $7671(2)$ & 7996(4) & $-118(2)$ & $37(1)$ \\
\hline$C(20)$ & $6949(3)$ & $4004(5)$ & $-204(2)$ & $50(1)$ \\
\hline$C(16)$ & $7593(2)$ & $2907(4)$ & $749(2)$ & $31(1)$ \\
\hline$C(5)$ & $10080(2)$ & 2109(4) & $2717(2)$ & $34(1)$ \\
\hline$C(33)$ & $7087(2)$ & $8093(4)$ & $72(2)$ & $39(1)$ \\
\hline$C(10)$ & $9515(2)$ & 2102(4) & $3041(2)$ & $38(1)$ \\
\hline$C(41)$ & $11935(2)$ & $6048(5)$ & $2672(3)$ & $56(1)$ \\
\hline$C(3)$ & $8264(2)$ & $754(4)$ & $1182(2)$ & $37(1)$ \\
\hline $\mathrm{C}(11)$ & $9349(3)$ & $3572(5)$ & $3200(2)$ & $52(1)$ \\
\hline$C(36)$ & $10930(2)$ & $7384(4)$ & $2889(2)$ & $39(1)$ \\
\hline$C(19)$ & $6451(3)$ & $4327(5)$ & $82(2)$ & $50(1)$ \\
\hline$C(13)$ & $10259(3)$ & $1935(5)$ & $1020(2)$ & $49(1)$ \\
\hline$C(40)$ & $11767(2)$ & $8518(4)$ & $2367(2)$ & $48(1)$ \\
\hline$C(18)$ & $6526(2)$ & $3929(5)$ & $696(2)$ & $44(1)$ \\
\hline C(39) & $10780(3)$ & $6072(5)$ & $3177(3)$ & $62(2)$ \\
\hline$C(8)$ & $11113(2)$ & $2237(4)$ & 2111(3) & $45(1)$ \\
\hline$C(38)$ & $11139(3)$ & $8518(5)$ & $3388(2)$ & $52(1)$ \\
\hline
\end{tabular}




\begin{tabular}{lcccc}
$\mathrm{C}(14)$ & $10332(3)$ & $3349(5)$ & $729(3)$ & $72(2)$ \\
$\mathrm{C}(15)$ & $10693(3)$ & $849(5)$ & $806(3)$ & $56(1)$ \\
$\mathrm{C}(12)$ & $9689(3)$ & $1198(5)$ & $3632(3)$ & $62(2)$ \\
$\mathrm{C}(24)$ & $6559(3)$ & $2064(6)$ & $1826(3)$ & $77(2)$ \\
$\mathrm{C}(23)$ & $7331(3)$ & $4108(6)$ & $2163(2)$ & $69(2)$ \\
$\mathrm{C}(1 \mathrm{X})$ & $10097(11)$ & $6300(20)$ & $4929(11)$ & $53(3)$ \\
$\mathrm{C}(2 \mathrm{X})$ & $9476(7)$ & $5762(15)$ & $4975(7)$ & $49(2)$ \\
$\mathrm{C}(3 \mathrm{X})$ & $9630(8)$ & $4598(13)$ & $5364(8)$ & $46(2)$ \\
$\mathrm{C}(4 \mathrm{X})$ & $9967(11)$ & $3457(19)$ & $5257(11)$ & $49(2)$ \\
$\mathrm{C}(5 \mathrm{X})$ & $9776(9)$ & $2181(15)$ & $5279(8)$ & $46(3)$ \\
$\mathrm{C}(1 \mathrm{Y})$ & $9700(15)$ & $6650(30)$ & $4878(15)$ & $53(4)$ \\
$\mathrm{C}(2 \mathrm{Y})$ & $10035(12)$ & $5420(20)$ & $5039(15)$ & $48(2)$ \\
$\mathrm{C}(3 \mathrm{Y})$ & $9570(15)$ & $4430(20)$ & $5122(15)$ & $48(2)$ \\
$\mathrm{C}(4 \mathrm{Y})$ & $9809(17)$ & $3130(30)$ & $5027(14)$ & $48(3)$ \\
$\mathrm{C}(5 \mathrm{Y})$ & $9892(19)$ & $2290(30)$ & $5514(14)$ & $48(4)$ \\
\hline
\end{tabular}

Table 3. Bond lengths $[\AA]$ and angles $\left[{ }^{\circ}\right]$ for $\mathbf{1} \bullet \mathbf{0 . 5}\left(\mathbf{C}_{\mathbf{5}} \mathbf{H}_{12}\right)$.

\begin{tabular}{llll}
\hline $\mathrm{Cu}(1)-\mathrm{C}(1)$ & $1.898(4)$ & $\mathrm{C}(17)-\mathrm{C}(22)$ & $1.520(6)$ \\
$\mathrm{Cu}(1)-\mathrm{C}(28)$ & $1.948(3)$ & $\mathrm{C}(29)-\mathrm{B}(1)$ & $1.579(6)$ \\
$\mathrm{C}(28)-\mathrm{C}(30)$ & $1.496(5)$ & $\mathrm{C}(22)-\mathrm{C}(23)$ & $1.518(7)$ \\
$\mathrm{C}(28)-\mathrm{C}(29)$ & $1.526(5)$ & $\mathrm{C}(22)-\mathrm{C}(24)$ & $1.526(7)$ \\
$\mathrm{N}(2)-\mathrm{C}(1)$ & $\mathrm{C}(35)-\mathrm{C}(34)$ & $1.379(6)$ \\
$\mathrm{N}(2)-\mathrm{C}(3)$ & $1.362(5)$ & $\mathrm{C}(21)-\mathrm{C}(20)$ & $1.383(6)$ \\
$\mathrm{N}(2)-\mathrm{C}(16)$ & $1.390(5)$ & $\mathrm{C}(21)-\mathrm{C}(16)$ & $1.397(6)$ \\
$\mathrm{N}(1)-\mathrm{C}(1)$ & $\mathrm{C}(21)-\mathrm{C}(25)$ & $1.518(7)$ \\
$\mathrm{N}(1)-\mathrm{C}(2)$ & $1.443(5)$ & $\mathrm{C}(25)-\mathrm{C}(26 \mathrm{~B})$ & $1.492(12)$ \\
$\mathrm{N}(1)-\mathrm{C}(4)$ & $1.360(5)$ & $\mathrm{C}(25)-\mathrm{C}(27 \mathrm{~A})$ & $1.517(10)$ \\
$\mathrm{C}(30)-\mathrm{C}(35)$ & $1.384(5)$ & $\mathrm{C}(25)-\mathrm{C}(27 \mathrm{~B})$ & $1.520(13)$ \\
$\mathrm{C}(30)-\mathrm{C}(31)$ & $1.439(5)$ & $\mathrm{C}(25)-\mathrm{C}(26 \mathrm{~A})$ & $1.583(9)$ \\
$\mathrm{C}(31)-\mathrm{C}(32)$ & $1.395(5)$ & $\mathrm{C}(37)-\mathrm{C}(40)$ & $1.523(6)$ \\
$\mathrm{O}(2)-\mathrm{B}(1)$ & $1.408(5)$ & $\mathrm{C}(37)-\mathrm{C}(41)$ & $1.525(6)$ \\
$\mathrm{O}(2)-\mathrm{C}(37)$ & $1.375(6)$ & $\mathrm{C}(37)-\mathrm{C}(36)$ & $1.533(6)$ \\
$\mathrm{O}(1)-\mathrm{B}(1)$ & $1.359(5)$ & $\mathrm{C}(2)-\mathrm{C}(3)$ & $1.340(6)$ \\
$\mathrm{O}(1)-\mathrm{C}(36)$ & $1.439(5)$ & $\mathrm{C}(9)-\mathrm{C}(4)$ & $1.392(6)$ \\
$\mathrm{C}(17)-\mathrm{C}(16)$ & $1.353(6)$ & $\mathrm{C}(9)-\mathrm{C}(8)$ & $1.396(6)$ \\
$\mathrm{C}(17)-\mathrm{C}(18)$ & $1.480(5)$ & $\mathrm{C}(9)-\mathrm{C}(13)$ & $1.516(7)$ \\
& $1.382(6)$ & &
\end{tabular}




\begin{tabular}{|c|c|c|c|}
\hline$C(7)-C(8)$ & $1.371(7)$ & $C(35)-C(30)-C(31)$ & $116.0(4)$ \\
\hline$C(7)-C(6)$ & $1.376(6)$ & $\mathrm{C}(35)-\mathrm{C}(30)-\mathrm{C}(28)$ & $122.4(3)$ \\
\hline$C(6)-C(5)$ & $1.393(6)$ & $\mathrm{C}(31)-\mathrm{C}(30)-\mathrm{C}(28)$ & $121.5(3)$ \\
\hline$C(32)-C(33)$ & $1.387(6)$ & $\mathrm{C}(32)-\mathrm{C}(31)-\mathrm{C}(30)$ & $121.7(4)$ \\
\hline$C(4)-C(5)$ & $1.399(6)$ & $\mathrm{B}(1)-\mathrm{O}(2)-\mathrm{C}(37)$ & $106.5(3)$ \\
\hline$C(34)-C(33)$ & $1.373(6)$ & $\mathrm{B}(1)-\mathrm{O}(1)-\mathrm{C}(36)$ & 106.6(3) \\
\hline$C(20)-C(19)$ & $1.374(7)$ & $\mathrm{C}(16)-\mathrm{C}(17)-\mathrm{C}(18)$ & $116.4(4)$ \\
\hline$C(5)-C(10)$ & $1.517(6)$ & $C(16)-C(17)-C(22)$ & $123.4(4)$ \\
\hline$C(10)-C(12)$ & $1.520(6)$ & $\mathrm{C}(18)-\mathrm{C}(17)-\mathrm{C}(22)$ & $120.2(4)$ \\
\hline$C(10)-C(11)$ & $1.529(6)$ & $\mathrm{C}(28)-\mathrm{C}(29)-\mathrm{B}(1)$ & $112.2(3)$ \\
\hline$C(36)-C(39)$ & $1.491(6)$ & $C(23)-C(22)-C(17)$ & $111.3(4)$ \\
\hline$C(36)-C(38)$ & $1.522(6)$ & $\mathrm{C}(23)-\mathrm{C}(22)-\mathrm{C}(24)$ & $110.6(4)$ \\
\hline$C(19)-C(18)$ & $1.368(7)$ & $C(17)-C(22)-C(24)$ & $112.7(4)$ \\
\hline$C(13)-C(15)$ & $1.534(6)$ & $\mathrm{C}(34)-\mathrm{C}(35)-\mathrm{C}(30)$ & $121.7(4)$ \\
\hline$C(13)-C(14)$ & $1.538(7)$ & $C(20)-C(21)-C(16)$ & $117.0(4)$ \\
\hline$C(1 X)-C(2 X)$ & $1.40(2)$ & $\mathrm{C}(20)-\mathrm{C}(21)-\mathrm{C}(25)$ & $120.8(4)$ \\
\hline$C(2 X)-C(3 X)$ & $1.395(15)$ & $C(16)-C(21)-C(25)$ & $122.2(4)$ \\
\hline$C(3 X)-C(4 X)$ & $1.358(16)$ & $C(26 B)-C(25)-C(27 A)$ & $126.6(11)$ \\
\hline$C(4 X)-C(5 X)$ & $1.302(19)$ & $C(26 \mathrm{~B})-\mathrm{C}(25)-\mathrm{C}(21)$ & $119.7(8)$ \\
\hline $\mathrm{C}(1 \mathrm{Y})-\mathrm{C}(2 \mathrm{Y})$ & $1.37(2)$ & $\mathrm{C}(27 \mathrm{~A})-\mathrm{C}(25)-\mathrm{C}(21)$ & 109.1(8) \\
\hline $\mathrm{C}(2 \mathrm{Y})-\mathrm{C}(3 \mathrm{Y})$ & $1.39(2)$ & $C(26 B)-C(25)-C(27 B)$ & $112.9(14)$ \\
\hline $\mathrm{C}(3 \mathrm{Y})-\mathrm{C}(4 \mathrm{Y})$ & $1.39(2)$ & $C(27 \mathrm{~A})-\mathrm{C}(25)-\mathrm{C}(27 \mathrm{~B})$ & $16.8(17)$ \\
\hline \multirow[t]{2}{*}{$\mathrm{C}(4 \mathrm{Y})-\mathrm{C}(5 \mathrm{Y})$} & $1.32(2)$ & $\mathrm{C}(21)-\mathrm{C}(25)-\mathrm{C}(27 \mathrm{~B})$ & $116.0(15)$ \\
\hline & & $C(26 B)-C(25)-C(26 A)$ & 37.1(9) \\
\hline $\mathrm{C}(1)-\mathrm{Cu}(1)-\mathrm{C}(28)$ & $175.07(16)$ & $C(27 A)-C(25)-C(26 A)$ & $108.0(7)$ \\
\hline $\mathrm{C}(30)-\mathrm{C}(28)-\mathrm{C}(29)$ & $115.8(3)$ & $C(21)-C(25)-C(26 A)$ & $109.7(6)$ \\
\hline $\mathrm{C}(30)-\mathrm{C}(28)-\mathrm{Cu}(1)$ & $104.5(2)$ & $C(27 B)-C(25)-C(26 A)$ & $91.2(14)$ \\
\hline $\mathrm{C}(29)-\mathrm{C}(28)-\mathrm{Cu}(1)$ & $114.9(3)$ & $\mathrm{O}(1)-\mathrm{B}(1)-\mathrm{O}(2)$ & $113.6(4)$ \\
\hline $\mathrm{C}(1)-\mathrm{N}(2)-\mathrm{C}(3)$ & $111.7(3)$ & $\mathrm{O}(1)-\mathrm{B}(1)-\mathrm{C}(29)$ & $122.1(4)$ \\
\hline $\mathrm{C}(1)-\mathrm{N}(2)-\mathrm{C}(16)$ & $122.5(3)$ & $\mathrm{O}(2)-\mathrm{B}(1)-\mathrm{C}(29)$ & $124.3(4)$ \\
\hline $\mathrm{C}(3)-\mathrm{N}(2)-\mathrm{C}(16)$ & $125.8(3)$ & $\mathrm{O}(2)-\mathrm{C}(37)-\mathrm{C}(40)$ & $106.8(4)$ \\
\hline $\mathrm{C}(1)-\mathrm{N}(1)-\mathrm{C}(2)$ & $111.5(3)$ & $\mathrm{O}(2)-\mathrm{C}(37)-\mathrm{C}(41)$ & $107.5(4)$ \\
\hline $\mathrm{C}(1)-\mathrm{N}(1)-\mathrm{C}(4)$ & $123.9(3)$ & $\mathrm{C}(40)-\mathrm{C}(37)-\mathrm{C}(41)$ & 109.1(4) \\
\hline $\mathrm{C}(2)-\mathrm{N}(1)-\mathrm{C}(4)$ & $124.6(3)$ & $\mathrm{O}(2)-\mathrm{C}(37)-\mathrm{C}(36)$ & $104.2(3)$ \\
\hline $\mathrm{N}(1)-\mathrm{C}(1)-\mathrm{N}(2)$ & $103.4(3)$ & $\mathrm{C}(40)-\mathrm{C}(37)-\mathrm{C}(36)$ & $113.8(4)$ \\
\hline $\mathrm{N}(1)-\mathrm{C}(1)-\mathrm{Cu}(1)$ & $131.0(3)$ & $C(41)-C(37)-C(36)$ & $114.9(4)$ \\
\hline $\mathrm{N}(2)-\mathrm{C}(1)-\mathrm{Cu}(1)$ & $125.6(3)$ & S36 $\mathrm{C}(3)-\mathrm{C}(2)-\mathrm{N}(1)$ & $107.1(3)$ \\
\hline
\end{tabular}




$\begin{array}{llll}\mathrm{C}(4)-\mathrm{C}(9)-\mathrm{C}(8) & 117.1(4) & \mathrm{C}(12)-\mathrm{C}(10)-\mathrm{C}(11) & 110.8(4) \\ \mathrm{C}(4)-\mathrm{C}(9)-\mathrm{C}(13) & 122.3(4) & \mathrm{C}(2)-\mathrm{C}(3)-\mathrm{N}(2) & 106.3(4) \\ \mathrm{C}(8)-\mathrm{C}(9)-\mathrm{C}(13) & 120.6(4) & \mathrm{O}(1)-\mathrm{C}(36)-\mathrm{C}(39) & 106.5(4) \\ \mathrm{C}(8)-\mathrm{C}(7)-\mathrm{C}(6) & 120.9(4) & \mathrm{O}(1)-\mathrm{C}(36)-\mathrm{C}(38) & 108.7(3) \\ \mathrm{C}(7)-\mathrm{C}(6)-\mathrm{C}(5) & 121.0(4) & \mathrm{C}(39)-\mathrm{C}(36)-\mathrm{C}(38) & 111.1(4) \\ \mathrm{C}(31)-\mathrm{C}(32)-\mathrm{C}(33) & 121.0(4) & \mathrm{O}(1)-\mathrm{C}(36)-\mathrm{C}(37) & 100.7(3) \\ \mathrm{C}(9)-\mathrm{C}(4)-\mathrm{C}(5) & 123.4(4) & \mathrm{C}(39)-\mathrm{C}(36)-\mathrm{C}(37) & 113.5(4) \\ \mathrm{C}(9)-\mathrm{C}(4)-\mathrm{N}(1) & 118.5(4) & \mathrm{C}(38)-\mathrm{C}(36)-\mathrm{C}(37) & 115.4(4) \\ \mathrm{C}(5)-\mathrm{C}(4)-\mathrm{N}(1) & 118.1(4) & \mathrm{C}(18)-\mathrm{C}(19)-\mathrm{C}(20) & 120.1(4) \\ \mathrm{C}(33)-\mathrm{C}(34)-\mathrm{C}(35) & 121.6(4) & \mathrm{C}(9)-\mathrm{C}(13)-\mathrm{C}(15) & 112.7(4) \\ \mathrm{C}(19)-\mathrm{C}(20)-\mathrm{C}(21) & 121.1(4) & \mathrm{C}(9)-\mathrm{C}(13)-\mathrm{C}(14) & 110.5(4) \\ \mathrm{C}(17)-\mathrm{C}(16)-\mathrm{C}(21) & 123.6(4) & \mathrm{C}(15)-\mathrm{C}(13)-\mathrm{C}(14) & 110.5(4) \\ \mathrm{C}(17)-\mathrm{C}(16)-\mathrm{N}(2) & 118.0(4) & \mathrm{C}(19)-\mathrm{C}(18)-\mathrm{C}(17) & 121.8(5) \\ \mathrm{C}(21)-\mathrm{C}(16)-\mathrm{N}(2) & 118.5(4) & \mathrm{C}(7)-\mathrm{C}(8)-\mathrm{C}(9) & 120.9(4) \\ \mathrm{C}(6)-\mathrm{C}(5)-\mathrm{C}(4) & 116.8(4) & \mathrm{C}(3 \mathrm{X})-\mathrm{C}(2 \mathrm{X})-\mathrm{C}(1 \mathrm{X}) & 107.7(14) \\ \mathrm{C}(6)-\mathrm{C}(5)-\mathrm{C}(10) & 121.5(4) & \mathrm{C}(4 \mathrm{X})-\mathrm{C}(3 \mathrm{X})-\mathrm{C}(2 \mathrm{X}) & 125.9(15) \\ \mathrm{C}(4)-\mathrm{C}(5)-\mathrm{C}(10) & 121.7(4) & \mathrm{C}(5 \mathrm{X})-\mathrm{C}(4 \mathrm{X})-\mathrm{C}(3 \mathrm{X}) & 126.6(19) \\ \mathrm{C}(34)-\mathrm{C}(33)-\mathrm{C}(32) & 117.9(4) & \mathrm{C}(1 \mathrm{Y})-\mathrm{C}(2 \mathrm{Y})-\mathrm{C}(3 \mathrm{Y}) & 109(2) \\ \mathrm{C}(5)-\mathrm{C}(10)-\mathrm{C}(12) & 112.6(4) & \mathrm{C}(4 \mathrm{Y})-\mathrm{C}(3 \mathrm{Y})-\mathrm{C}(2 \mathrm{Y}) & 109(2) \\ \mathrm{C}(5)-\mathrm{C}(10)-\mathrm{C}(11) & 110.5(3) & \mathrm{C}(5 \mathrm{Y})-\mathrm{C}(4 \mathrm{Y})-\mathrm{C}(3 \mathrm{Y}) & 114(2) \\ & & & \\ & & & \end{array}$

Table 4. Anisotropic displacement parameters $\left(\AA^{2} \times 10^{3}\right)$ for $\mathbf{1 \bullet 0 . 5}\left(\mathbf{C}_{\mathbf{5}} \mathbf{H}_{\mathbf{1 2}}\right)$. The anisotropic displacement factor exponent takes the form: $-2 \pi^{2}\left[\mathrm{~h}^{2} \mathrm{a}^{* 2} \mathrm{U}^{11}+\ldots+2 \mathrm{~h} \mathrm{k} \mathrm{a}^{*} \mathrm{~b}^{*} \mathrm{U}^{12}\right]$

\begin{tabular}{lllllll}
\hline & $\mathrm{U}^{11}$ & $\mathrm{U}^{22}$ & $\mathrm{U}^{33}$ & $\mathrm{U}^{23}$ & $\mathrm{U}^{13}$ & $\mathrm{U}^{12}$ \\
\hline $\mathrm{Cu}(1)$ & $27(1)$ & $16(1)$ & $32(1)$ & $2(1)$ & $6(1)$ & $-1(1)$ \\
$\mathrm{C}(28)$ & $27(2)$ & $19(2)$ & $27(2)$ & $-1(2)$ & $12(2)$ & $-3(2)$ \\
$\mathrm{N}(2)$ & $33(2)$ & $20(2)$ & $37(2)$ & $0(1)$ & $9(2)$ & $-3(1)$ \\
$\mathrm{N}(1)$ & $28(2)$ & $16(2)$ & $42(2)$ & $5(1)$ & $9(2)$ & $2(1)$ \\
$\mathrm{C}(1)$ & $26(2)$ & $20(2)$ & $36(2)$ & $2(2)$ & $14(2)$ & $-1(2)$ \\
$\mathrm{C}(30)$ & $27(2)$ & $12(2)$ & $33(2)$ & $-3(2)$ & $14(2)$ & $-5(1)$ \\
$\mathrm{C}(31)$ & $37(2)$ & $23(2)$ & $34(2)$ & $5(2)$ & $12(2)$ & $-3(2)$ \\
$\mathrm{O}(2)$ & $39(2)$ & $51(2)$ & $47(2)$ & $-6(2)$ & $14(2)$ & $-2(2)$ \\
$\mathrm{O}(1)$ & $37(2)$ & $51(2)$ & $43(2)$ & $-6(2)$ & $7(2)$ & $11(1)$ \\
$\mathrm{C}(17)$ & $32(2)$ & $27(2)$ & $37(2)$ & $-1(2)$ & & \\
& & & & & & \\
\end{tabular}




\begin{tabular}{|c|c|c|c|c|c|c|}
\hline $\mathrm{C}(29)$ & $42(3)$ & $29(2)$ & $37(2)$ & $0(2)$ & $12(2)$ & $2(2)$ \\
\hline$C(22)$ & $34(2)$ & $45(3)$ & $35(2)$ & $5(2)$ & $6(2)$ & $4(2)$ \\
\hline$C(35)$ & $34(2)$ & $28(2)$ & $34(2)$ & $-2(2)$ & $12(2)$ & $-1(2)$ \\
\hline$C(21)$ & $47(3)$ & $33(2)$ & $36(3)$ & $-6(2)$ & $3(2)$ & $-13(2)$ \\
\hline$C(25)$ & $67(3)$ & $64(3)$ & $42(3)$ & $-2(2)$ & $17(3)$ & $-8(3)$ \\
\hline$C(26 A)$ & $85(9)$ & $47(4)$ & $73(7)$ & $-13(4)$ & $46(7)$ & $-19(5)$ \\
\hline$C(27 \mathrm{~A})$ & $138(12)$ & $35(5)$ & $70(8)$ & $12(5)$ & $61(9)$ & $14(6)$ \\
\hline$C(26 B)$ & $49(13)$ & 71(11) & $83(17)$ & $-1(11)$ & $32(12)$ & $-3(8)$ \\
\hline $\mathrm{C}(27 \mathrm{~B})$ & $120(20)$ & $39(14)$ & $64(17)$ & $-7(13)$ & $14(13)$ & $16(11)$ \\
\hline $\mathrm{B}(1)$ & $33(3)$ & $30(2)$ & $44(3)$ & $-2(2)$ & $21(2)$ & $-1(2)$ \\
\hline$C(37)$ & $38(2)$ & $26(2)$ & $39(3)$ & $0(2)$ & $6(2)$ & $-3(2)$ \\
\hline $\mathrm{C}(2)$ & $47(3)$ & $15(2)$ & $52(3)$ & $3(2)$ & $16(2)$ & $-1(2)$ \\
\hline $\mathrm{C}(9)$ & $38(3)$ & $25(2)$ & $54(3)$ & $9(2)$ & $21(2)$ & $8(2)$ \\
\hline$C(7)$ & $27(2)$ & $27(2)$ & $75(4)$ & $8(2)$ & $10(2)$ & $0(2)$ \\
\hline$C(6)$ & $38(3)$ & $25(2)$ & $53(3)$ & $6(2)$ & $6(2)$ & $1(2)$ \\
\hline$C(32)$ & $25(2)$ & $37(2)$ & $53(3)$ & $3(2)$ & $14(2)$ & $-1(2)$ \\
\hline C(4) & $30(2)$ & $18(2)$ & $44(3)$ & $9(2)$ & $9(2)$ & $2(2)$ \\
\hline$C(34)$ & $38(3)$ & $39(2)$ & $30(2)$ & $-2(2)$ & $4(2)$ & $-4(2)$ \\
\hline$C(20)$ & $59(3)$ & $42(3)$ & $36(3)$ & $11(2)$ & $-8(3)$ & $-20(2)$ \\
\hline$C(16)$ & $34(2)$ & $18(2)$ & $34(2)$ & $-1(2)$ & $0(2)$ & $-6(2)$ \\
\hline $\mathrm{C}(5)$ & $32(2)$ & $17(2)$ & $54(3)$ & $7(2)$ & $12(2)$ & $1(2)$ \\
\hline$C(33)$ & $31(2)$ & $36(2)$ & $42(3)$ & $2(2)$ & $-1(2)$ & $-1(2)$ \\
\hline$C(10)$ & $30(2)$ & $41(2)$ & $43(3)$ & $6(2)$ & $11(2)$ & $-5(2)$ \\
\hline$C(41)$ & $44(3)$ & $40(3)$ & $84(4)$ & $3(3)$ & $21(3)$ & $6(2)$ \\
\hline $\mathrm{C}(3)$ & $44(3)$ & $18(2)$ & $47(3)$ & $-5(2)$ & $13(2)$ & $-3(2)$ \\
\hline$C(11)$ & $61(3)$ & $46(3)$ & $55(3)$ & $8(2)$ & $30(3)$ & $10(2)$ \\
\hline$C(36)$ & $41(3)$ & $31(2)$ & $42(3)$ & $-4(2)$ & $9(2)$ & $-6(2)$ \\
\hline$C(19)$ & $46(3)$ & $35(2)$ & $56(3)$ & $12(2)$ & $-7(3)$ & $-2(2)$ \\
\hline$C(13)$ & $49(3)$ & $51(3)$ & $56(3)$ & $7(2)$ & $28(3)$ & $7(2)$ \\
\hline$C(40)$ & $47(3)$ & $35(2)$ & $62(3)$ & $5(2)$ & $16(2)$ & $-7(2)$ \\
\hline$C(18)$ & $38(3)$ & $40(3)$ & $49(3)$ & $2(2)$ & $4(2)$ & $3(2)$ \\
\hline$C(39)$ & $88(4)$ & $49(3)$ & $64(4)$ & $-5(3)$ & $44(3)$ & $-16(3)$ \\
\hline$C(8)$ & $34(3)$ & $34(2)$ & $71(4)$ & $11(2)$ & $24(2)$ & $4(2)$ \\
\hline$C(38)$ & $74(4)$ & $38(3)$ & $42(3)$ & $-10(2)$ & $12(3)$ & $-1(2)$ \\
\hline$C(14)$ & $107(5)$ & $53(3)$ & $75(4)$ & $23(3)$ & $56(4)$ & $27(3)$ \\
\hline$C(15)$ & $60(3)$ & $42(3)$ & $74(4)$ & $0(3)$ & $35(3)$ & $-2(2)$ \\
\hline$C(12)$ & $63(4)$ & $58(3)$ & $73(4)$ & ${ }^{31(3)}$ S38 & $31(3)$ & $5(3)$ \\
\hline
\end{tabular}




\begin{tabular}{lllllll}
$\mathrm{C}(24)$ & $84(4)$ & $85(4)$ & $54(4)$ & $21(3)$ & $8(3)$ & $-36(4)$ \\
$\mathrm{C}(23)$ & $98(5)$ & $61(3)$ & $45(3)$ & $-5(3)$ & $19(3)$ & $-15(3)$ \\
$\mathrm{C}(1 \mathrm{X})$ & $50(6)$ & $61(7)$ & $47(7)$ & $-5(6)$ & $11(6)$ & $-14(5)$ \\
$\mathrm{C}(2 \mathrm{X})$ & $40(5)$ & $58(5)$ & $46(5)$ & $-1(4)$ & $9(5)$ & $-5(4)$ \\
$\mathrm{C}(3 \mathrm{X})$ & $37(4)$ & $58(4)$ & $42(5)$ & $-2(4)$ & $11(5)$ & $-4(4)$ \\
$\mathrm{C}(4 \mathrm{X})$ & $39(5)$ & $61(4)$ & $45(6)$ & $-1(4)$ & $8(5)$ & $-3(4)$ \\
$\mathrm{C}(5 \mathrm{X})$ & $34(6)$ & $60(5)$ & $41(7)$ & $10(6)$ & $5(6)$ & $4(5)$ \\
$\mathrm{C}(1 \mathrm{Y})$ & $46(7)$ & $60(6)$ & $48(7)$ & $0(6)$ & $7(7)$ & $-3(6)$ \\
$\mathrm{C}(2 \mathrm{Y})$ & $39(5)$ & $58(5)$ & $45(5)$ & $-1(5)$ & $11(5)$ & $-4(4)$ \\
$\mathrm{C}(3 \mathrm{Y})$ & $38(5)$ & $59(4)$ & $46(5)$ & $0(4)$ & $11(5)$ & $-3(4)$ \\
$\mathrm{C}(4 \mathrm{Y})$ & $38(5)$ & $60(5)$ & $45(6)$ & $-1(5)$ & $10(5)$ & $-4(4)$ \\
$\mathrm{C}(5 \mathrm{Y})$ & $35(7)$ & $63(6)$ & $44(8)$ & $0(6)$ & $10(7)$ & $0(6)$ \\
\hline
\end{tabular}

Table 5. Hydrogen coordinates $\left(\mathrm{x} 10^{4}\right)$ and isotropic displacement parameters $\left(\AA^{2} \times 10^{3}\right)$ for $1 \bullet 0.5\left(\mathrm{C}_{5} \mathrm{H}_{12}\right)$.

\begin{tabular}{|c|c|c|c|c|}
\hline & $\mathrm{x}$ & $\mathrm{y}$ & $\mathrm{z}$ & $\mathrm{U}(\mathrm{eq})$ \\
\hline $\mathrm{H}(28)$ & 9029 & 7139 & 1807 & 28 \\
\hline $\mathrm{H}(31)$ & 7755 & 7024 & 1527 & 37 \\
\hline $\mathrm{H}(29 \mathrm{~A})$ & 9583 & 8226 & 1059 & 43 \\
\hline $\mathrm{H}(29 B)$ & 9761 & 6681 & 905 & 43 \\
\hline $\mathrm{H}(22)$ & 7587 & 2212 & 1887 & 46 \\
\hline $\mathrm{H}(35)$ & 8684 & 7527 & 147 & 38 \\
\hline $\mathrm{H}(25 \mathrm{~A})$ & 8537 & 2816 & 129 & 69 \\
\hline $\mathrm{H}(25 \mathrm{~B})$ & 8411 & 2381 & 143 & 69 \\
\hline $\mathrm{H}(26 \mathrm{~A})$ & 8479 & 3873 & -887 & 95 \\
\hline $\mathrm{H}(26 \mathrm{~B})$ & 8237 & 4982 & -457 & 95 \\
\hline $\mathrm{H}(26 \mathrm{C})$ & 7678 & 4188 & -1012 & 95 \\
\hline $\mathrm{H}(27 \mathrm{~A})$ & 8234 & 1354 & -774 & 112 \\
\hline $\mathrm{H}(27 \mathrm{~B})$ & 7438 & 1723 & -898 & 112 \\
\hline $\mathrm{H}(27 \mathrm{C})$ & 7838 & 857 & -281 & 112 \\
\hline $\mathrm{H}(26 \mathrm{D})$ & 8865 & 3626 & -521 & 98 \\
\hline $\mathrm{H}(26 \mathrm{E})$ & 8790 & 4463 & 84 & 98 \\
\hline $\mathrm{H}(26 \mathrm{~F})$ & 8259 & 4727 & -604 & 98 \\
\hline $\mathrm{H}(27 \mathrm{D})$ & 8262 & 1709 & $\mathrm{~S} 39^{-904}$ & 117 \\
\hline
\end{tabular}




\begin{tabular}{|c|c|c|c|c|}
\hline $\mathrm{H}(27 \mathrm{E})$ & 7481 & 2227 & -1073 & 117 \\
\hline $\mathrm{H}(27 \mathrm{~F})$ & 7744 & 1009 & -568 & 117 \\
\hline $\mathrm{H}(2)$ & 9102 & -357 & 1693 & 45 \\
\hline $\mathrm{H}(7)$ & 11738 & 2494 & 3004 & 53 \\
\hline $\mathrm{H}(6)$ & 10890 & 2380 & 3512 & 48 \\
\hline $\mathrm{H}(32)$ & 6730 & 7749 & 832 & 45 \\
\hline $\mathrm{H}(34)$ & 7650 & 8227 & -544 & 45 \\
\hline $\mathrm{H}(20)$ & 6889 & 4276 & -632 & 60 \\
\hline $\mathrm{H}(33)$ & 6666 & 8411 & -211 & 47 \\
\hline $\mathrm{H}(10)$ & 9092 & 1715 & 2732 & 46 \\
\hline $\mathrm{H}(41 \mathrm{~A})$ & 11698 & 5164 & 2660 & 84 \\
\hline $\mathrm{H}(41 \mathrm{~B})$ & 12226 & 6227 & 3106 & 84 \\
\hline $\mathrm{H}(41 \mathrm{C})$ & 12221 & 6016 & 2382 & 84 \\
\hline $\mathrm{H}(3)$ & 7919 & 84 & 1007 & 44 \\
\hline $\mathrm{H}(11 \mathrm{~A})$ & 9743 & 3955 & 3528 & 77 \\
\hline $\mathrm{H}(11 \mathrm{~B})$ & 8946 & 3560 & 3358 & 77 \\
\hline $\mathrm{H}(11 \mathrm{C})$ & 9252 & 4145 & 2816 & 77 \\
\hline $\mathrm{H}(19)$ & 6054 & 4827 & -147 & 60 \\
\hline $\mathrm{H}(13)$ & 9766 & 1649 & 855 & 59 \\
\hline $\mathrm{H}(40 \mathrm{~A})$ & 12009 & 8358 & 2048 & 72 \\
\hline $\mathrm{H}(40 \mathrm{~B})$ & 12098 & 8792 & 2771 & 72 \\
\hline $\mathrm{H}(40 \mathrm{C})$ & 11426 & 9252 & 2220 & 72 \\
\hline $\mathrm{H}(18)$ & 6176 & 4154 & 886 & 53 \\
\hline $\mathrm{H}(39 \mathrm{~A})$ & 10422 & 6232 & 3385 & 93 \\
\hline $\mathrm{H}(39 \mathrm{~B})$ & 11199 & 5744 & 3492 & 93 \\
\hline $\mathrm{H}(39 \mathrm{C})$ & 10622 & 5376 & 2842 & 93 \\
\hline $\mathrm{H}(8)$ & 11469 & 2285 & 1911 & 54 \\
\hline $\mathrm{H}(38 \mathrm{~A})$ & 11151 & 9403 & 3177 & 78 \\
\hline $\mathrm{H}(38 \mathrm{~B})$ & 11596 & 8314 & 3678 & 78 \\
\hline $\mathrm{H}(38 \mathrm{C})$ & 10804 & 8566 & 3630 & 78 \\
\hline $\mathrm{H}(14 \mathrm{~A})$ & 10033 & 4019 & 853 & 109 \\
\hline $\mathrm{H}(14 \mathrm{~B})$ & 10198 & 3271 & 264 & 109 \\
\hline $\mathrm{H}(14 \mathrm{C})$ & 10811 & 3657 & 886 & 109 \\
\hline $\mathrm{H}(15 \mathrm{~A})$ & 11181 & 1097 & 967 & 83 \\
\hline $\mathrm{H}(15 \mathrm{~B})$ & 10557 & 812 & 340 & 83 \\
\hline $\mathrm{H}(15 \mathrm{C})$ & 10620 & -55 & 974 & 83 \\
\hline $\mathrm{H}(12 \mathrm{~A})$ & 9759 & 246 & $\mathrm{~S} 40^{3514}$ & 93 \\
\hline
\end{tabular}




\begin{tabular}{|c|c|c|c|c|}
\hline $\mathrm{H}(12 \mathrm{~B})$ & 9310 & 1227 & 3823 & 93 \\
\hline $\mathrm{H}(12 \mathrm{C})$ & 10111 & 1537 & 3939 & 93 \\
\hline $\mathrm{H}(24 \mathrm{~A})$ & 6156 & 2671 & 1712 & 116 \\
\hline $\mathrm{H}(24 \mathrm{~B})$ & 6655 & 1772 & 2270 & 116 \\
\hline $\mathrm{H}(24 \mathrm{C})$ & 6468 & 1251 & 1548 & 116 \\
\hline $\mathrm{H}(23 \mathrm{~A})$ & 7753 & 4549 & 2131 & 103 \\
\hline $\mathrm{H}(23 \mathrm{~B})$ & 7390 & 3838 & 2606 & 103 \\
\hline $\mathrm{H}(23 \mathrm{C})$ & 6946 & 4758 & 2026 & 103 \\
\hline $\mathrm{H}(1 \mathrm{X} 1)$ & 10010 & 7174 & 4693 & 80 \\
\hline $\mathrm{H}(1 \mathrm{X} 2)$ & 10310 & 5646 & 4704 & 80 \\
\hline H(1X3) & 10408 & 6467 & 5357 & 80 \\
\hline $\mathrm{H}(2 \mathrm{X} 1)$ & 9234 & 6455 & 5162 & 59 \\
\hline $\mathrm{H}(2 \mathrm{X} 2)$ & 9175 & 5508 & 4548 & 59 \\
\hline $\mathrm{H}(3 \mathrm{X} 1)$ & 9894 & 4937 & 5791 & 55 \\
\hline $\mathrm{H}(3 \mathrm{X} 2)$ & 9184 & 4266 & 5406 & 55 \\
\hline $\mathrm{H}(4 \mathrm{X} 1)$ & 10022 & 3582 & 4827 & 59 \\
\hline $\mathrm{H}(4 \mathrm{X} 2)$ & 10435 & 3517 & 5559 & 59 \\
\hline $\mathrm{H}(5 \mathrm{X} 1)$ & 10081 & 1574 & 5128 & 69 \\
\hline $\mathrm{H}(5 \mathrm{X} 2)$ & 9302 & 2069 & 5006 & 69 \\
\hline $\mathrm{H}(5 \mathrm{X} 3)$ & 9800 & 1941 & 5718 & 69 \\
\hline H(1Y1) & 9907 & 7164 & 4595 & 79 \\
\hline $\mathrm{H}(1 \mathrm{Y} 2)$ & 9742 & 7194 & 5264 & 79 \\
\hline H(1Y3) & 9213 & 6482 & 4660 & 79 \\
\hline $\mathrm{H}(2 \mathrm{Y} 1)$ & 10416 & 5522 & 5438 & 57 \\
\hline $\mathrm{H}(2 \mathrm{Y} 2)$ & 10233 & 5120 & 4698 & 57 \\
\hline H(3Y1) & 9525 & 4497 & 5558 & 58 \\
\hline H(3Y2) & 9112 & 4592 & 4814 & 58 \\
\hline $\mathrm{H}(4 \mathrm{Y} 1)$ & 9480 & 2713 & 4648 & 58 \\
\hline $\mathrm{H}(4 \mathrm{Y} 2)$ & 10255 & 3237 & 4934 & 58 \\
\hline $\mathrm{H}(5 \mathrm{Y} 1)$ & 10123 & 1448 & 5438 & 71 \\
\hline $\mathrm{H}(5 \mathrm{Y} 2)$ & 9442 & 2054 & 5564 & 71 \\
\hline H(5Y3) & 10173 & 2741 & 5902 & 71 \\
\hline
\end{tabular}




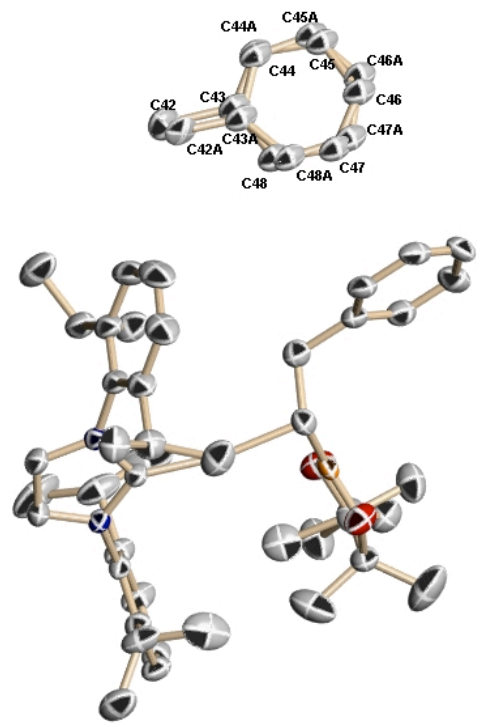

Figure S3. X-ray crystal structure of $\mathbf{2} \bullet \mathbf{0 . 5} \mathbf{C}_{7} \mathbf{H}_{\mathbf{8}}$ shown as $50 \%$ ellipsoids. For clarity, hydrogen atoms have been omitted.

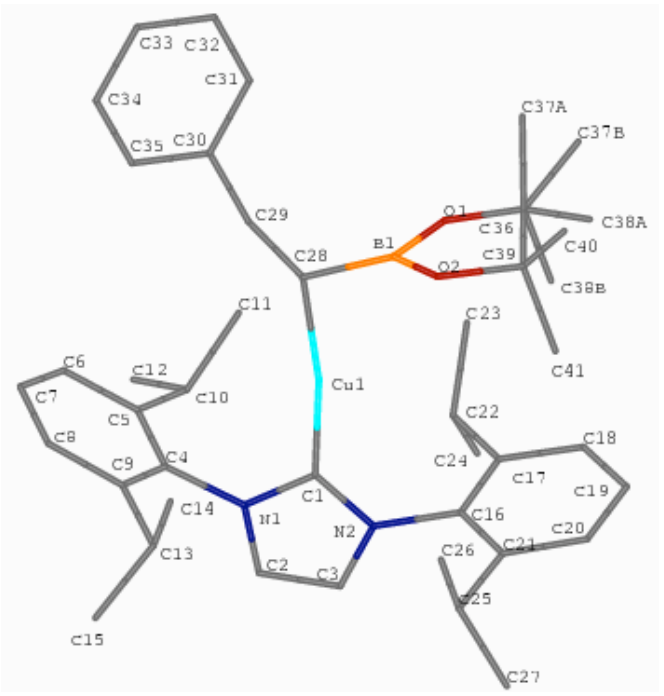

Figure S4. X-ray crystal structure of $\mathbf{2 \bullet 0 . 5 C _ { 7 }} \mathbf{H}_{\mathbf{8}}$ shown as a stick figure for clarity in labeling. Solvent and hydrogen atoms have been omitted for clarity.

\section{[1,3-Bis(2,6-diisopropylphenyl)imidazol-2-ylidene]copper(I) (1-pinacolato)-2-phenylethyl (2).}

Two methyl groups of the pinacolborane were disordered $(\mathrm{C}(37)$, and $\mathrm{C}(38))$ and were refined over two positions with the help of similarity restraints on 1-2 and 1-3 distances and displacement parameters. Rigid bond restraints for anisotropic displacement parameters were also used. A toluene molecule located on a special position was also disordered and was refined with the aid of part -1 and part -2 instructions, as well as the restraints mentioned above. The relative occupancies for the disordered parts 
were refined freely, while constraining the overall occupation to unity. One peak of significant residual electron density $\left(3.20 \mathrm{e} / \AA^{3}\right)$ remained upon final refinement which was less than $1 \AA$ away from copper. This peak corresponds to a disorder involving $\mathrm{Cu}(1)$ and a different orientation of the boroalkyl group. The disorder represents less than $10 \%$ of the occupancy for those atoms, and attempts to include it in the model were unsuccessful yielding mathematically unstable refinements even with strong restraints.

Table S2. Crystal data and structure refinement for $\mathbf{2} \bullet \mathbf{0 . 5} \mathbf{C}_{7} \mathbf{H}_{\mathbf{8}}$.

Identification code

Empirical formula

Formula weight

Temperature

Wavelength

Crystal system

Space group

Unit cell dimensions

Volume

$\mathrm{Z}$

Density (calculated)

Absorption coefficient

$\mathrm{F}(000)$

Crystal size

Theta range for data collection

Index ranges

Reflections collected

Independent reflections

Completeness to $\theta=26.37^{\circ}$

Absorption correction

Max. and min. transmission

Refinement method

Data / restraints / parameters

Goodness-of-fit on $\mathrm{F}^{2}$
05159

$\mathrm{C}_{44.50} \mathrm{H}_{60} \mathrm{BCuN}_{2} \mathrm{O}_{2}$

729.29

100(2) K

$0.71073 \AA$

Monoclinic

$\mathrm{P} 2(1) / \mathrm{n}$

$\mathrm{a}=12.5994(8) \AA \quad \alpha=90^{\circ}$.

$\mathrm{b}=19.7548(15) \AA \quad \beta=110.025(2)^{\circ}$.

$\mathrm{c}=17.9349(14) \AA \quad \gamma=90^{\circ}$.

4194.1(5) $\AA^{3}$

4

$1.155 \mathrm{Mg} / \mathrm{m}^{3}$

$0.557 \mathrm{~mm}^{-1}$

1564

$0.21 \times 0.10 \times 0.10 \mathrm{~mm}^{3}$

1.59 to $26.37^{\circ}$.

$-15<=\mathrm{h}<=15,-24<=\mathrm{k}<=24,-22<=1<=22$

75308

$8592[\mathrm{R}(\mathrm{int})=0.0409]$

$100.0 \%$

Semi-empirical from equivalents

0.9464 and 0.8920

Full-matrix least-squares on $\mathrm{F}^{2}$

8592 / $251 / 586$

1.029 
Final R indices $[\mathrm{I}>2 \sigma(\mathrm{I})]$

$\mathrm{R}$ indices (all data)

Largest diff. peak and hole
$\mathrm{R} 1=0.0671, \mathrm{wR} 2=0.1578$

$\mathrm{R} 1=0.0789, \mathrm{wR} 2=0.1672$

3.205 and $-1.747 \mathrm{e} / \AA^{3}$

Table 2. Atomic coordinates $\left(\times 10^{4}\right)$ and equivalent isotropic displacement parameters $\left(\AA^{2} \times 10^{3}\right)$ for $\mathbf{2} \bullet \mathbf{0 . 5} \mathbf{C}_{7} \mathbf{H}_{\mathbf{8}}$. $\mathrm{U}(\mathrm{eq})$ is defined as one third of the trace of the orthogonalized $\mathrm{U}^{\mathrm{ij}}$ tensor.

\begin{tabular}{|c|c|c|c|c|}
\hline & $\mathrm{x}$ & $\mathrm{y}$ & $\mathrm{z}$ & $\mathrm{U}(\mathrm{eq})$ \\
\hline $\mathrm{Cu}(1 \mathrm{~A})$ & $524(1)$ & $2230(1)$ & 5991(1) & $28(1)$ \\
\hline$C(28)$ & $1705(3)$ & $1725(2)$ & $6801(2)$ & $30(1)$ \\
\hline$C(29)$ & $2347(3)$ & $1313(2)$ & $6366(2)$ & $35(1)$ \\
\hline $\mathrm{C}(1)$ & $-430(2)$ & $2716(1)$ & $5104(2)$ & $21(1)$ \\
\hline $\mathrm{N}(1)$ & $-52(2)$ & $3026(1)$ & $4564(1)$ & $23(1)$ \\
\hline $\mathrm{N}(2)$ & $-1557(2)$ & $2831(1)$ & $4817(1)$ & $22(1)$ \\
\hline$C(2)$ & $-926(3)$ & $3321(2)$ & $3960(2)$ & $29(1)$ \\
\hline$C(3)$ & $-1877(3)$ & $3199(2)$ & $4113(2)$ & $29(1)$ \\
\hline$C(4)$ & $1118(3)$ & $3004(2)$ & $4622(2)$ & $27(1)$ \\
\hline$C(5)$ & $1452(3)$ & $2496(2)$ & 4199(2) & $32(1)$ \\
\hline$C(6)$ & $2589(3)$ & $2463(2)$ & $4294(2)$ & $43(1)$ \\
\hline$C(7)$ & $3352(3)$ & $2912(2)$ & $4779(3)$ & $49(1)$ \\
\hline $\mathrm{C}(8)$ & 2998(3) & $3413(2)$ & $5175(2)$ & $44(1)$ \\
\hline $\mathrm{C}(9)$ & $1863(3)$ & $3470(2)$ & $5105(2)$ & $32(1)$ \\
\hline$C(10)$ & $618(3)$ & 1981(2) & $3686(2)$ & $37(1)$ \\
\hline $\mathrm{C}(11)$ & $641(3)$ & $1336(2)$ & $4156(2)$ & $41(1)$ \\
\hline$C(12)$ & $827(5)$ & $1824(2)$ & $2910(2)$ & $54(1)$ \\
\hline$C(13)$ & $1500(3)$ & $4012(2)$ & $5567(2)$ & $37(1)$ \\
\hline$C(14)$ & $2059(4)$ & $3896(2)$ & $6455(2)$ & $51(1)$ \\
\hline$C(15)$ & $1717(4)$ & $4722(2)$ & $5321(2)$ & $45(1)$ \\
\hline$C(16)$ & $-2349(2)$ & $2526(2)$ & $5138(2)$ & $23(1)$ \\
\hline $\mathrm{C}(17)$ & $-2723(3)$ & $1875(2)$ & $4895(2)$ & $33(1)$ \\
\hline $\mathrm{C}(18)$ & $-3501(3)$ & $1586(2)$ & $5198(2)$ & $38(1)$ \\
\hline$C(19)$ & $-3876(3)$ & $1938(2)$ & $5719(2)$ & $33(1)$ \\
\hline$C(20)$ & $-3490(3)$ & $2584(2)$ & $5953(2)$ & $33(1)$ \\
\hline$C(21)$ & $-2710(3)$ & $2897(2)$ & $5671(2)$ & $28(1)$ \\
\hline$C(22)$ & $-2339(4)$ & $1478(2)$ & $4303(2)$ & $49(1)$ \\
\hline$C(23)$ & $-1878(4)$ & $782(2)$ & $4621(3)$ & $63(1)$ \\
\hline
\end{tabular}




\begin{tabular}{|c|c|c|c|c|}
\hline$C(24)$ & $-3316(5)$ & $1433(3)$ & $3506(3)$ & $75(2)$ \\
\hline$C(25)$ & $-2247(4)$ & $3597(2)$ & $5963(2)$ & $43(1)$ \\
\hline$C(26)$ & $-1299(4)$ & $3535(2)$ & $6761(3)$ & $63(1)$ \\
\hline $\mathrm{C}(27)$ & $-3152(4)$ & $4083(2)$ & $6022(3)$ & $51(1)$ \\
\hline $\mathrm{B}(1)$ & $920(3)$ & $1361(2)$ & $7150(2)$ & $28(1)$ \\
\hline $\mathrm{O}(1)$ & $277(2)$ & $785(1)$ & $6849(2)$ & $42(1)$ \\
\hline $\mathrm{O}(2)$ & $709(2)$ & 1594(1) & $7806(1)$ & $40(1)$ \\
\hline$C(36)$ & $-295(3)$ & $598(2)$ & $7379(2)$ & $38(1)$ \\
\hline$C(37 A)$ & $465(4)$ & $15(2)$ & $7874(3)$ & $48(1)$ \\
\hline $\mathrm{C}(38 \mathrm{~A})$ & $-1432(4)$ & 297(3) & $6926(3)$ & $54(2)$ \\
\hline $\mathrm{C}(37 \mathrm{~B})$ & $-490(20)$ & $-60(9)$ & $7629(13)$ & $45(6)$ \\
\hline $\mathrm{C}(38 \mathrm{~B})$ & $-1421(15)$ & $843(11)$ & $6653(13)$ & $49(7)$ \\
\hline $\mathrm{C}(39)$ & $-231(3)$ & $1239(2)$ & $7884(2)$ & $35(1)$ \\
\hline$C(40)$ & $-20(6)$ & $1102(3)$ & $8750(3)$ & $84(2)$ \\
\hline $\mathrm{C}(41)$ & $-1256(4)$ & $1690(3)$ & $7507(4)$ & $72(2)$ \\
\hline$C(30)$ & $3487(3)$ & $1081(2)$ & $6923(2)$ & $32(1)$ \\
\hline $\mathrm{C}(31)$ & $3597(3)$ & $488(2)$ & $7336(2)$ & $34(1)$ \\
\hline$C(32)$ & $4637(3)$ & $266(2)$ & $7840(2)$ & $39(1)$ \\
\hline$C(33)$ & $5596(3)$ & $654(2)$ & $7947(2)$ & $39(1)$ \\
\hline$C(34)$ & $5506(3)$ & $1261(2)$ & $7550(2)$ & $34(1)$ \\
\hline$C(35)$ & $4459(3)$ & $1474(2)$ & $7043(2)$ & $33(1)$ \\
\hline$C(42)$ & $3750(30)$ & $637(14)$ & $3710(20)$ & $44(4)$ \\
\hline$C(43)$ & $4530(30)$ & $202(15)$ & $4435(19)$ & $32(3)$ \\
\hline$C(44)$ & $5280(60)$ & $-260(30)$ & $4390(50)$ & $31(3)$ \\
\hline$C(45)$ & $6020(30)$ & $-579(18)$ & $5120(20)$ & $31(3)$ \\
\hline$C(46)$ & $5860(30)$ & $-390(18)$ & $5817(17)$ & $32(3)$ \\
\hline$C(47)$ & $5040(30)$ & $57(18)$ & $5826(19)$ & $31(3)$ \\
\hline $\mathrm{C}(48)$ & $4370(30)$ & $350(19)$ & $5150(20)$ & $32(3)$ \\
\hline $\mathrm{C}(42 \mathrm{~A})$ & $3890(30)$ & $665(16)$ & $3960(30)$ & $34(4)$ \\
\hline$C(43 A)$ & $4520(30)$ & $276(18)$ & $4577(18)$ & $31(3)$ \\
\hline $\mathrm{C}(44 \mathrm{~A})$ & $5300(70)$ & $-190(30)$ & $4430(60)$ & $31(3)$ \\
\hline$C(45 A)$ & $5970(30)$ & $-600(20)$ & $4959(18)$ & $31(3)$ \\
\hline$C(46 A)$ & $5980(30)$ & $-545(17)$ & $5720(20)$ & $32(3)$ \\
\hline $\mathrm{C}(47 \mathrm{~A})$ & $5280(30)$ & $-76(19)$ & $5946(16)$ & $31(3)$ \\
\hline $\mathrm{C}(48 \mathrm{~A})$ & $4530(40)$ & $320(30)$ & $5340(30)$ & $31(3)$ \\
\hline
\end{tabular}


Table 3. Bond lengths $[\AA]$ and angles $\left[{ }^{\circ}\right]$ for $\mathbf{2} \cdot \mathbf{0 . 5} \mathbf{C}_{7} \mathbf{H}_{\mathbf{8}}$.

\begin{tabular}{|c|c|c|c|}
\hline $\mathrm{Cu}(1 \mathrm{~A})-\mathrm{C}(1)$ & $1.895(3)$ & $C(25)-C(27)$ & $1.521(5)$ \\
\hline $\mathrm{Cu}(1 \mathrm{~A})-\mathrm{C}(28)$ & $1.959(3)$ & $C(25)-C(26)$ & $1.523(6)$ \\
\hline $\mathrm{C}(28)-\mathrm{B}(1)$ & $1.520(5)$ & $\mathrm{B}(1)-\mathrm{O}(2)$ & $1.371(4)$ \\
\hline$C(28)-C(29)$ & $1.536(5)$ & $\mathrm{B}(1)-\mathrm{O}(1)$ & $1.393(5)$ \\
\hline$C(29)-C(30)$ & $1.512(4)$ & $\mathrm{O}(1)-\mathrm{C}(36)$ & $1.424(4)$ \\
\hline $\mathrm{C}(1)-\mathrm{N}(2)$ & $1.354(4)$ & $\mathrm{O}(2)-\mathrm{C}(39)$ & $1.423(4)$ \\
\hline $\mathrm{C}(1)-\mathrm{N}(1)$ & $1.362(4)$ & $\mathrm{C}(36)-\mathrm{C}(37 \mathrm{~B})$ & $1.424(15)$ \\
\hline $\mathrm{N}(1)-\mathrm{C}(2)$ & $1.383(4)$ & $C(36)-C(38 A)$ & $1.507(5)$ \\
\hline $\mathrm{N}(1)-\mathrm{C}(4)$ & $1.442(4)$ & $C(36)-C(39)$ & $1.543(5)$ \\
\hline $\mathrm{N}(2)-\mathrm{C}(3)$ & $1.391(4)$ & $\mathrm{C}(36)-\mathrm{C}(37 \mathrm{~A})$ & $1.566(6)$ \\
\hline $\mathrm{N}(2)-\mathrm{C}(16)$ & $1.444(4)$ & $\mathrm{C}(36)-\mathrm{C}(38 \mathrm{~B})$ & $1.635(16)$ \\
\hline$C(2)-C(3)$ & $1.338(5)$ & $C(39)-C(40)$ & $1.508(5)$ \\
\hline $\mathrm{C}(4)-\mathrm{C}(9)$ & $1.386(5)$ & $C(39)-C(41)$ & $1.523(6)$ \\
\hline$C(4)-C(5)$ & $1.406(5)$ & $\mathrm{C}(30)-\mathrm{C}(31)$ & $1.367(5)$ \\
\hline$C(5)-C(6)$ & $1.385(5)$ & $C(30)-C(35)$ & $1.403(5)$ \\
\hline$C(5)-C(10)$ & $1.525(5)$ & $C(31)-C(32)$ & $1.384(5)$ \\
\hline$C(6)-C(7)$ & $1.376(6)$ & $C(32)-C(33)$ & $1.388(5)$ \\
\hline$C(7)-C(8)$ & $1.378(6)$ & $\mathrm{C}(33)-\mathrm{C}(34)$ & $1.379(5)$ \\
\hline $\mathrm{C}(8)-\mathrm{C}(9)$ & $1.397(5)$ & $C(34)-C(35)$ & $1.387(5)$ \\
\hline $\mathrm{C}(9)-\mathrm{C}(13)$ & $1.517(5)$ & $C(42)-C(43)$ & $1.59(4)$ \\
\hline$C(10)-C(11)$ & $1.523(5)$ & $\mathrm{C}(43)-\mathrm{C}(44)$ & $1.32(6)$ \\
\hline$C(10)-C(12)$ & $1.533(5)$ & $C(43)-C(48)$ & $1.41(3)$ \\
\hline$C(13)-C(14)$ & $1.522(5)$ & $C(44)-C(45)$ & $1.46(10)$ \\
\hline$C(13)-C(15)$ & $1.524(5)$ & $C(45)-C(46)$ & $1.38(3)$ \\
\hline$C(16)-C(17)$ & $1.389(5)$ & $C(46)-C(47)$ & $1.36(3)$ \\
\hline$C(16)-C(21)$ & $1.399(4)$ & $C(47)-C(48)$ & $1.34(3)$ \\
\hline$C(17)-C(18)$ & $1.395(4)$ & $C(42 A)-C(43 A)$ & $1.36(5)$ \\
\hline$C(17)-C(22)$ & $1.525(5)$ & $C(43 A)-C(48 A)$ & $1.36(4)$ \\
\hline$C(18)-C(19)$ & $1.372(5)$ & $C(43 \mathrm{~A})-\mathrm{C}(44 \mathrm{~A})$ & $1.44(7)$ \\
\hline$C(19)-C(20)$ & $1.378(5)$ & $C(44 A)-C(45 A)$ & $1.31(11)$ \\
\hline$C(20)-C(21)$ & $1.394(4)$ & $C(45 A)-C(46 A)$ & $1.37(3)$ \\
\hline$C(21)-C(25)$ & $1.522(5)$ & $C(46 A)-C(47 A)$ & $1.43(3)$ \\
\hline$C(22)-C(23)$ & $1.525(7)$ & $C(47 \mathrm{~A})-\mathrm{C}(48 \mathrm{~A})$ & $1.41(3)$ \\
\hline$C(22)-C(24)$ & $1.536(7)$ & & \\
\hline
\end{tabular}




\begin{tabular}{|c|c|c|c|}
\hline $\mathrm{C}(1)-\mathrm{Cu}(1 \mathrm{~A})-\mathrm{C}(28)$ & $169.51(13)$ & $C(16)-C(17)-C(18)$ & $117.7(3)$ \\
\hline $\mathrm{B}(1)-\mathrm{C}(28)-\mathrm{C}(29)$ & 119.3(3) & $C(16)-C(17)-C(22)$ & $122.7(3)$ \\
\hline $\mathrm{B}(1)-\mathrm{C}(28)-\mathrm{Cu}(1 \mathrm{~A})$ & $96.3(2)$ & $\mathrm{C}(18)-\mathrm{C}(17)-\mathrm{C}(22)$ & $119.6(3)$ \\
\hline $\mathrm{C}(29)-\mathrm{C}(28)-\mathrm{Cu}(1 \mathrm{~A})$ & $106.9(2)$ & $\mathrm{C}(19)-\mathrm{C}(18)-\mathrm{C}(17)$ & $120.7(3)$ \\
\hline $\mathrm{C}(30)-\mathrm{C}(29)-\mathrm{C}(28)$ & $111.8(3)$ & $C(18)-C(19)-C(20)$ & $120.6(3)$ \\
\hline $\mathrm{N}(2)-\mathrm{C}(1)-\mathrm{N}(1)$ & $103.4(2)$ & $C(19)-C(20)-C(21)$ & 121.3(3) \\
\hline $\mathrm{N}(2)-\mathrm{C}(1)-\mathrm{Cu}(1 \mathrm{~A})$ & $133.3(2)$ & $C(20)-C(21)-C(16)$ & $116.8(3)$ \\
\hline $\mathrm{N}(1)-\mathrm{C}(1)-\mathrm{Cu}(1 \mathrm{~A})$ & $123.3(2)$ & $C(20)-C(21)-C(25)$ & $120.8(3)$ \\
\hline$C(1)-N(1)-C(2)$ & $111.6(2)$ & $C(16)-C(21)-C(25)$ & $122.3(3)$ \\
\hline $\mathrm{C}(1)-\mathrm{N}(1)-\mathrm{C}(4)$ & $122.3(2)$ & $C(23)-C(22)-C(17)$ & $112.0(3)$ \\
\hline $\mathrm{C}(2)-\mathrm{N}(1)-\mathrm{C}(4)$ & $126.1(2)$ & $\mathrm{C}(23)-\mathrm{C}(22)-\mathrm{C}(24)$ & $112.2(4)$ \\
\hline $\mathrm{C}(1)-\mathrm{N}(2)-\mathrm{C}(3)$ & $111.9(2)$ & $\mathrm{C}(17)-\mathrm{C}(22)-\mathrm{C}(24)$ & $109.5(4)$ \\
\hline $\mathrm{C}(1)-\mathrm{N}(2)-\mathrm{C}(16)$ & $123.8(2)$ & $C(27)-C(25)-C(21)$ & $113.0(3)$ \\
\hline$C(3)-N(2)-C(16)$ & $123.8(2)$ & $\mathrm{C}(27)-\mathrm{C}(25)-\mathrm{C}(26)$ & $110.6(3)$ \\
\hline $\mathrm{C}(3)-\mathrm{C}(2)-\mathrm{N}(1)$ & $107.0(3)$ & $\mathrm{C}(21)-\mathrm{C}(25)-\mathrm{C}(26)$ & 109.6(3) \\
\hline $\mathrm{C}(2)-\mathrm{C}(3)-\mathrm{N}(2)$ & $106.2(3)$ & $\mathrm{O}(2)-\mathrm{B}(1)-\mathrm{O}(1)$ & $110.4(3)$ \\
\hline$C(9)-C(4)-C(5)$ & $123.2(3)$ & $\mathrm{O}(2)-\mathrm{B}(1)-\mathrm{C}(28)$ & $122.6(3)$ \\
\hline $\mathrm{C}(9)-\mathrm{C}(4)-\mathrm{N}(1)$ & $118.8(3)$ & $\mathrm{O}(1)-\mathrm{B}(1)-\mathrm{C}(28)$ & $126.9(3)$ \\
\hline $\mathrm{C}(5)-\mathrm{C}(4)-\mathrm{N}(1)$ & $118.0(3)$ & $\mathrm{B}(1)-\mathrm{O}(1)-\mathrm{C}(36)$ & 108.2(3) \\
\hline$C(6)-C(5)-C(4)$ & 117.1(3) & $\mathrm{B}(1)-\mathrm{O}(2)-\mathrm{C}(39)$ & 109.3(3) \\
\hline$C(6)-C(5)-C(10)$ & $120.8(3)$ & $\mathrm{C}(37 \mathrm{~B})-\mathrm{C}(36)-\mathrm{O}(1)$ & $128.9(10)$ \\
\hline$C(4)-C(5)-C(10)$ & $122.0(3)$ & $C(37 B)-C(36)-C(38 A)$ & $64.8(10)$ \\
\hline$C(7)-C(6)-C(5)$ & $121.1(4)$ & $\mathrm{O}(1)-\mathrm{C}(36)-\mathrm{C}(38 \mathrm{~A})$ & $110.3(3)$ \\
\hline$C(6)-C(7)-C(8)$ & $120.6(3)$ & $\mathrm{C}(37 \mathrm{~B})-\mathrm{C}(36)-\mathrm{C}(39)$ & $123.0(10)$ \\
\hline $\mathrm{C}(7)-\mathrm{C}(8)-\mathrm{C}(9)$ & $120.8(4)$ & $\mathrm{O}(1)-\mathrm{C}(36)-\mathrm{C}(39)$ & $104.0(3)$ \\
\hline$C(4)-C(9)-C(8)$ & $117.1(3)$ & $\mathrm{C}(38 \mathrm{~A})-\mathrm{C}(36)-\mathrm{C}(39)$ & $119.3(4)$ \\
\hline$C(4)-C(9)-C(13)$ & $122.9(3)$ & $\mathrm{C}(37 \mathrm{~B})-\mathrm{C}(36)-\mathrm{C}(37 \mathrm{~A})$ & $44.5(10)$ \\
\hline $\mathrm{C}(8)-\mathrm{C}(9)-\mathrm{C}(13)$ & $119.9(3)$ & $\mathrm{O}(1)-\mathrm{C}(36)-\mathrm{C}(37 \mathrm{~A})$ & $102.7(3)$ \\
\hline $\mathrm{C}(11)-\mathrm{C}(10)-\mathrm{C}(5)$ & $110.2(3)$ & $\mathrm{C}(38 \mathrm{~A})-\mathrm{C}(36)-\mathrm{C}(37 \mathrm{~A})$ & $107.4(4)$ \\
\hline$C(11)-C(10)-C(12)$ & $110.9(3)$ & $C(39)-C(36)-C(37 A)$ & $111.9(3)$ \\
\hline$C(5)-C(10)-C(12)$ & $112.7(3)$ & $\mathrm{C}(37 \mathrm{~B})-\mathrm{C}(36)-\mathrm{C}(38 \mathrm{~B})$ & $108.9(12)$ \\
\hline $\mathrm{C}(9)-\mathrm{C}(13)-\mathrm{C}(14)$ & $110.3(3)$ & $\mathrm{O}(1)-\mathrm{C}(36)-\mathrm{C}(38 \mathrm{~B})$ & $83.7(9)$ \\
\hline $\mathrm{C}(9)-\mathrm{C}(13)-\mathrm{C}(15)$ & $112.0(3)$ & $\mathrm{C}(38 \mathrm{~A})-\mathrm{C}(36)-\mathrm{C}(38 \mathrm{~B})$ & $44.1(8)$ \\
\hline $\mathrm{C}(14)-\mathrm{C}(13)-\mathrm{C}(15)$ & $112.2(3)$ & $\mathrm{C}(39)-\mathrm{C}(36)-\mathrm{C}(38 \mathrm{~B})$ & $94.7(9)$ \\
\hline$C(17)-C(16)-C(21)$ & $123.0(3)$ & $\mathrm{C}(37 \mathrm{~A})-\mathrm{C}(36)-\mathrm{C}(38 \mathrm{~B})$ & $149.8(8)$ \\
\hline $\mathrm{C}(17)-\mathrm{C}(16)-\mathrm{N}(2)$ & $117.9(3)$ & $\mathrm{O}(2)-\mathrm{C}(39)-\mathrm{C}(40)$ & $109.3(4)$ \\
\hline $\mathrm{C}(21)-\mathrm{C}(16)-\mathrm{N}(2)$ & $119.0(3)$ & $\mathrm{S} 47^{\mathrm{O}(2)-\mathrm{C}(39)-\mathrm{C}(41)}$ & $106.1(3)$ \\
\hline
\end{tabular}




$\begin{array}{llll}\mathrm{C}(40)-\mathrm{C}(39)-\mathrm{C}(41) & 112.7(4) & \mathrm{C}(48)-\mathrm{C}(43)-\mathrm{C}(42) & 113(3) \\ \mathrm{O}(2)-\mathrm{C}(39)-\mathrm{C}(36) & 103.0(3) & \mathrm{C}(43)-\mathrm{C}(44)-\mathrm{C}(45) & 120(6) \\ \mathrm{C}(40)-\mathrm{C}(39)-\mathrm{C}(36) & 114.3(4) & \mathrm{C}(46)-\mathrm{C}(45)-\mathrm{C}(44) & 116(4) \\ \mathrm{C}(41)-\mathrm{C}(39)-\mathrm{C}(36) & 110.7(3) & \mathrm{C}(47)-\mathrm{C}(46)-\mathrm{C}(45) & 122(3) \\ \mathrm{C}(31)-\mathrm{C}(30)-\mathrm{C}(35) & 118.0(3) & \mathrm{C}(48)-\mathrm{C}(47)-\mathrm{C}(46) & 121(3) \\ \mathrm{C}(31)-\mathrm{C}(30)-\mathrm{C}(29) & 121.0(3) & \mathrm{C}(47)-\mathrm{C}(48)-\mathrm{C}(43) & 119(3) \\ \mathrm{C}(35)-\mathrm{C}(30)-\mathrm{C}(29) & 121.0(3) & \mathrm{C}(48 \mathrm{~A})-\mathrm{C}(43 \mathrm{~A})-\mathrm{C}(42 \mathrm{~A}) & 126(3) \\ \mathrm{C}(30)-\mathrm{C}(31)-\mathrm{C}(32) & 121.6(3) & \mathrm{C}(48 \mathrm{~A})-\mathrm{C}(43 \mathrm{~A})-\mathrm{C}(44 \mathrm{~A}) & 117(4) \\ \mathrm{C}(31)-\mathrm{C}(32)-\mathrm{C}(33) & 120.0(3) & \mathrm{C}(42 \mathrm{~A})-\mathrm{C}(43 \mathrm{~A})-\mathrm{C}(44 \mathrm{~A}) & 117(5) \\ \mathrm{C}(34)-\mathrm{C}(33)-\mathrm{C}(32) & 119.6(3) & \mathrm{C}(45 \mathrm{~A})-\mathrm{C}(44 \mathrm{~A})-\mathrm{C}(43 \mathrm{~A}) & 125(8) \\ \mathrm{C}(33)-\mathrm{C}(34)-\mathrm{C}(35) & 119.6(3) & \mathrm{C}(44 \mathrm{~A})-\mathrm{C}(45 \mathrm{~A})-\mathrm{C}(46 \mathrm{~A}) & 118(5) \\ \mathrm{C}(34)-\mathrm{C}(35)-\mathrm{C}(30) & 121.2(3) & \mathrm{C}(45 \mathrm{~A})-\mathrm{C}(46 \mathrm{~A})-\mathrm{C}(47 \mathrm{~A}) & 122(3) \\ \mathrm{C}(44)-\mathrm{C}(43)-\mathrm{C}(48) & 122(4) & \mathrm{C}(48 \mathrm{~A})-\mathrm{C}(47 \mathrm{~A})-\mathrm{C}(46 \mathrm{~A}) & 117(3) \\ \mathrm{C}(44)-\mathrm{C}(43)-\mathrm{C}(42) & 125(4) & \mathrm{C}(43 \mathrm{~A})-\mathrm{C}(48 \mathrm{~A})-\mathrm{C}(47 \mathrm{~A}) & 121(4)\end{array}$

Table 4. Anisotropic displacement parameters $\left(\AA^{2} \times 10^{3}\right)$ for $\mathbf{2} \bullet \mathbf{0 . 5} \mathbf{C}_{\mathbf{7}} \mathbf{H}_{\mathbf{8}}$. The anisotropic displacement factor exponent takes the form: $-2 \pi^{2}\left[h^{2} a^{* 2} U^{11}+\ldots+2 h k a^{*} b^{*} U^{12}\right]$

\begin{tabular}{lllllll}
\hline & $\mathrm{U}^{11}$ & $\mathrm{U}^{22}$ & $\mathrm{U}^{33}$ & $\mathrm{U}^{23}$ & $\mathrm{U}^{13}$ & $\mathrm{U}^{12}$ \\
\hline $\mathrm{Cu}(1 \mathrm{~A})$ & $22(1)$ & $37(1)$ & $29(1)$ & $13(1)$ & $12(1)$ & $5(1)$ \\
$\mathrm{C}(28)$ & $24(2)$ & $37(2)$ & $29(2)$ & $4(1)$ & $7(1)$ & $4(1)$ \\
$\mathrm{C}(29)$ & $30(2)$ & $45(2)$ & $30(2)$ & $1(2)$ & $11(1)$ & $2(2)$ \\
$\mathrm{C}(1)$ & $23(1)$ & $22(1)$ & $21(1)$ & $3(1)$ & $10(1)$ & $1(1)$ \\
$\mathrm{N}(1)$ & $26(1)$ & $23(1)$ & $24(1)$ & $4(1)$ & $14(1)$ & $2(1)$ \\
$\mathrm{N}(2)$ & $22(1)$ & $28(1)$ & $19(1)$ & $4(1)$ & $8(1)$ & $1(1)$ \\
$\mathrm{C}(2)$ & $35(2)$ & $31(2)$ & $23(1)$ & $8(1)$ & $13(1)$ & $5(1)$ \\
$\mathrm{C}(3)$ & $29(2)$ & $37(2)$ & $22(1)$ & $8(1)$ & $9(1)$ & $6(1)$ \\
$\mathrm{C}(4)$ & $28(2)$ & $30(2)$ & $29(2)$ & $8(1)$ & $17(1)$ & $2(1)$ \\
$\mathrm{C}(5)$ & $38(2)$ & $34(2)$ & $33(2)$ & $9(1)$ & $23(1)$ & $7(1)$ \\
$\mathrm{C}(6)$ & $44(2)$ & $43(2)$ & $56(2)$ & $10(2)$ & $35(2)$ & $10(2)$ \\
$\mathrm{C}(7)$ & $34(2)$ & $55(2)$ & $71(3)$ & $20(2)$ & $34(2)$ & $6(2)$ \\
$\mathrm{C}(8)$ & $31(2)$ & $48(2)$ & $56(2)$ & $9(2)$ & $20(2)$ & $-6(2)$ \\
$\mathrm{C}(9)$ & $31(2)$ & $34(2)$ & $37(2)$ & $7(1)$ & $18(1)$ & $0(1)$ \\
$\mathrm{C}(10)$ & $46(2)$ & $36(2)$ & $35(2)$ & $1(1)$ & $23(2)$ & $8(2)$ \\
$\mathrm{C}(11)$ & $49(2)$ & $40(2)$ & $42(2)$ & $2(2)$ & $25(2)$ & $-4(2)$ \\
$\mathrm{C}(12)$ & $94(3)$ & $38(2)$ & $46(2)$ & $0(2)$ & & $42(2)$ \\
& & & & & & $7(2)$ \\
& & & & & \\
\end{tabular}




\begin{tabular}{|c|c|c|c|c|c|c|}
\hline$C(13)$ & $34(2)$ & $36(2)$ & $42(2)$ & $-4(2)$ & $16(2)$ & $-7(1)$ \\
\hline$C(14)$ & $69(3)$ & $44(2)$ & $44(2)$ & $2(2)$ & $26(2)$ & $-4(2)$ \\
\hline$C(15)$ & $51(2)$ & $37(2)$ & $42(2)$ & $-1(2)$ & $10(2)$ & $-6(2)$ \\
\hline$C(16)$ & $18(1)$ & $33(2)$ & $18(1)$ & $4(1)$ & $5(1)$ & $-1(1)$ \\
\hline$C(17)$ & $31(2)$ & $41(2)$ & $28(2)$ & $-8(1)$ & $14(1)$ & $-10(1)$ \\
\hline$C(18)$ & $35(2)$ & $47(2)$ & $35(2)$ & $-12(2)$ & $17(2)$ & $-20(2)$ \\
\hline$C(19)$ & $24(2)$ & $48(2)$ & $29(2)$ & $-1(1)$ & $12(1)$ & $-10(1)$ \\
\hline$C(20)$ & $30(2)$ & $44(2)$ & $29(2)$ & $-1(1)$ & $17(1)$ & $-3(1)$ \\
\hline $\mathrm{C}(21)$ & $27(2)$ & $33(2)$ & $26(2)$ & $2(1)$ & $12(1)$ & $-2(1)$ \\
\hline$C(22)$ & 61(3) & $50(2)$ & $52(2)$ & $-25(2)$ & $40(2)$ & $-30(2)$ \\
\hline$C(23)$ & $73(3)$ & $49(3)$ & $92(4)$ & $-30(2)$ & $59(3)$ & $-24(2)$ \\
\hline$C(24)$ & $104(4)$ & $89(4)$ & $45(2)$ & $-35(3)$ & $43(3)$ & $-47(3)$ \\
\hline$C(25)$ & $60(2)$ & $33(2)$ & $52(2)$ & $-7(2)$ & $41(2)$ & $-8(2)$ \\
\hline$C(26)$ & $46(2)$ & $55(3)$ & $86(3)$ & $-34(2)$ & $18(2)$ & $-22(2)$ \\
\hline$C(27)$ & $76(3)$ & $40(2)$ & $50(2)$ & $-5(2)$ & $36(2)$ & $4(2)$ \\
\hline $\mathrm{B}(1)$ & $19(2)$ & $34(2)$ & $27(2)$ & $10(1)$ & $5(1)$ & $9(1)$ \\
\hline $\mathrm{O}(1)$ & $54(2)$ & $36(1)$ & $45(1)$ & $-5(1)$ & $28(1)$ & $-2(1)$ \\
\hline $\mathrm{O}(2)$ & $37(1)$ & $54(2)$ & $30(1)$ & $-9(1)$ & $13(1)$ & $-16(1)$ \\
\hline$C(36)$ & $32(2)$ & $45(2)$ & $36(2)$ & $6(2)$ & $9(1)$ & $-11(2)$ \\
\hline$C(37 A)$ & $45(3)$ & $43(3)$ & $48(3)$ & $15(2)$ & $7(2)$ & $-3(2)$ \\
\hline$C(38 A)$ & $37(3)$ & $52(3)$ & $63(3)$ & $11(2)$ & $6(2)$ & $-13(2)$ \\
\hline$C(37 B)$ & $54(16)$ & $47(13)$ & $38(12)$ & $0(10)$ & $20(11)$ & $-3(11)$ \\
\hline $\mathrm{C}(38 \mathrm{~B})$ & $55(15)$ & $31(12)$ & $65(16)$ & $-8(11)$ & $24(12)$ & $-19(10)$ \\
\hline$C(39)$ & $30(2)$ & $48(2)$ & $32(2)$ & $6(2)$ & $17(1)$ & $4(2)$ \\
\hline$C(40)$ & $132(5)$ & $89(4)$ & $43(3)$ & $4(3)$ & $46(3)$ & $-4(4)$ \\
\hline$C(41)$ & $45(3)$ & $67(3)$ & $107(4)$ & $1(3)$ & $31(3)$ & $13(2)$ \\
\hline$C(30)$ & $27(2)$ & $46(2)$ & $27(2)$ & $1(1)$ & $15(1)$ & $7(1)$ \\
\hline$C(31)$ & $32(2)$ & $40(2)$ & $31(2)$ & $-6(1)$ & $14(1)$ & $0(1)$ \\
\hline$C(32)$ & $41(2)$ & $31(2)$ & $47(2)$ & $0(2)$ & $19(2)$ & $12(2)$ \\
\hline$C(33)$ & $26(2)$ & $51(2)$ & $36(2)$ & $-2(2)$ & $7(1)$ & $17(2)$ \\
\hline$C(34)$ & $26(2)$ & $44(2)$ & $35(2)$ & $-12(2)$ & $15(1)$ & $0(1)$ \\
\hline$C(35)$ & $35(2)$ & $38(2)$ & $36(2)$ & $4(1)$ & $23(2)$ & $7(1)$ \\
\hline$C(42)$ & $51(8)$ & 49(8) & $29(8)$ & $-15(8)$ & $9(8)$ & $1(6)$ \\
\hline$C(43)$ & $36(5)$ & $35(5)$ & $29(7)$ & $-13(5)$ & $15(5)$ & $-11(4)$ \\
\hline$C(44)$ & $33(5)$ & $36(6)$ & $29(6)$ & $-14(5)$ & $15(5)$ & $-12(4)$ \\
\hline$C(45)$ & $31(5)$ & $38(5)$ & $29(7)$ & $-15(5)$ & $15(5)$ & $-10(4)$ \\
\hline$C(46)$ & $33(5)$ & $38(6)$ & $28(6)$ & $-17(5)$ S49 & $12(5)$ & $-10(4)$ \\
\hline
\end{tabular}




\begin{tabular}{lllllll}
$\mathrm{C}(47)$ & $33(6)$ & $36(7)$ & $28(6)$ & $-15(5)$ & $16(5)$ & $-11(5)$ \\
$\mathrm{C}(48)$ & $35(6)$ & $34(6)$ & $29(7)$ & $-14(5)$ & $15(5)$ & $-11(4)$ \\
$\mathrm{C}(42 \mathrm{~A})$ & $41(8)$ & $38(8)$ & $23(9)$ & $-24(8)$ & $9(9)$ & $-5(5)$ \\
$\mathrm{C}(43 \mathrm{~A})$ & $35(5)$ & $34(5)$ & $28(7)$ & $-15(5)$ & $16(5)$ & $-11(4)$ \\
$\mathrm{C}(44 \mathrm{~A})$ & $32(5)$ & $36(6)$ & $28(6)$ & $-14(5)$ & $16(5)$ & $-12(4)$ \\
$\mathrm{C}(45 \mathrm{~A})$ & $31(5)$ & $36(5)$ & $29(7)$ & $-16(5)$ & $13(5)$ & $-12(4)$ \\
$\mathrm{C}(46 \mathrm{~A})$ & $33(5)$ & $37(6)$ & $27(6)$ & $-16(5)$ & $12(6)$ & $-11(4)$ \\
$\mathrm{C}(47 \mathrm{~A})$ & $33(6)$ & $36(7)$ & $28(6)$ & $-15(4)$ & $15(5)$ & $-13(5)$ \\
$\mathrm{C}(48 \mathrm{~A})$ & $34(6)$ & $35(6)$ & $29(7)$ & $-15(5)$ & $16(5)$ & $-12(4)$ \\
\hline
\end{tabular}

Table 5. Hydrogen coordinates ( $\left.x 10^{4}\right)$ and isotropic displacement parameters $\left(\AA^{2} \times 10^{3}\right)$ for $2 \cdot 0.5 \mathrm{C}_{7} \mathrm{H}_{8}$.

\begin{tabular}{|c|c|c|c|c|}
\hline & $\mathrm{x}$ & $\mathrm{y}$ & z & $\mathrm{U}(\mathrm{eq})$ \\
\hline $\mathrm{H}(28)$ & 2224 & 2038 & 7199 & 36 \\
\hline $\mathrm{H}(29 \mathrm{~A})$ & 1892 & 913 & 6115 & 42 \\
\hline $\mathrm{H}(29 \mathrm{~B})$ & 2454 & 1594 & 5940 & 42 \\
\hline $\mathrm{H}(2)$ & -865 & 3565 & 3520 & 35 \\
\hline $\mathrm{H}(3)$ & -2620 & 3335 & 3802 & 35 \\
\hline $\mathrm{H}(6)$ & 2846 & 2125 & 4020 & 51 \\
\hline $\mathrm{H}(7)$ & 4129 & 2876 & 4841 & 59 \\
\hline $\mathrm{H}(8)$ & 3534 & 3725 & 5499 & 52 \\
\hline $\mathrm{H}(10)$ & -156 & 2180 & 3543 & 44 \\
\hline $\mathrm{H}(11 \mathrm{~A})$ & 1395 & 1132 & 4312 & 62 \\
\hline $\mathrm{H}(11 \mathrm{~B})$ & 82 & 1016 & 3827 & 62 \\
\hline $\mathrm{H}(11 \mathrm{C})$ & 460 & 1445 & 4633 & 62 \\
\hline $\mathrm{H}(12 \mathrm{~A})$ & 881 & 2249 & 2643 & 81 \\
\hline $\mathrm{H}(12 \mathrm{~B})$ & 200 & 1553 & 2564 & 81 \\
\hline $\mathrm{H}(12 \mathrm{C})$ & 1534 & 1570 & 3028 & 81 \\
\hline $\mathrm{H}(13)$ & 667 & 3964 & 5440 & 44 \\
\hline $\mathrm{H}(14 \mathrm{~A})$ & 1870 & 3442 & 6591 & 76 \\
\hline $\mathrm{H}(14 \mathrm{~B})$ & 1787 & 4234 & 6746 & 76 \\
\hline $\mathrm{H}(14 \mathrm{C})$ & 2880 & 3936 & 6600 & 76 \\
\hline $\mathrm{H}(15 \mathrm{~A})$ & 2527 & 4781 & 5421 & 67 \\
\hline $\mathrm{H}(15 \mathrm{~B})$ & 1466 & 5057 & 5629 & 67 \\
\hline $\mathrm{H}(15 \mathrm{C})$ & 1297 & 4787 & $\mathrm{~S} 50^{4754}$ & 67 \\
\hline
\end{tabular}




\begin{tabular}{|c|c|c|c|c|}
\hline $\mathrm{H}(18)$ & -3774 & 1141 & 5042 & 45 \\
\hline $\mathrm{H}(19)$ & -4407 & 1735 & 5920 & 39 \\
\hline $\mathrm{H}(20)$ & -3761 & 2820 & 6314 & 39 \\
\hline $\mathrm{H}(22)$ & -1714 & 1738 & 4212 & 59 \\
\hline $\mathrm{H}(23 \mathrm{~A})$ & -1261 & 834 & 5129 & 95 \\
\hline $\mathrm{H}(23 \mathrm{~B})$ & -1595 & 556 & 4240 & 95 \\
\hline $\mathrm{H}(23 \mathrm{C})$ & -2482 & 510 & 4698 & 95 \\
\hline $\mathrm{H}(24 \mathrm{~A})$ & -3934 & 1168 & 3574 & 112 \\
\hline $\mathrm{H}(24 \mathrm{~B})$ & -3050 & 1213 & 3113 & 112 \\
\hline $\mathrm{H}(24 \mathrm{C})$ & -3586 & 1890 & 3324 & 112 \\
\hline $\mathrm{H}(25)$ & -1919 & 3789 & 5574 & 51 \\
\hline $\mathrm{H}(26 \mathrm{~A})$ & -1596 & 3337 & 7151 & 95 \\
\hline $\mathrm{H}(26 \mathrm{~B})$ & -987 & 3984 & 6941 & 95 \\
\hline $\mathrm{H}(26 \mathrm{C})$ & -702 & 3242 & 6704 & 95 \\
\hline $\mathrm{H}(27 \mathrm{~A})$ & -3781 & 4091 & 5515 & 77 \\
\hline $\mathrm{H}(27 \mathrm{~B})$ & -2830 & 4539 & 6144 & 77 \\
\hline $\mathrm{H}(27 \mathrm{C})$ & -3427 & 3933 & 6444 & 77 \\
\hline $\mathrm{H}(37 \mathrm{~A})$ & 506 & -352 & 7518 & 72 \\
\hline $\mathrm{H}(37 \mathrm{~B})$ & 138 & -158 & 8260 & 72 \\
\hline $\mathrm{H}(37 \mathrm{C})$ & 1226 & 189 & 8154 & 72 \\
\hline $\mathrm{H}(38 \mathrm{~A})$ & -1858 & 610 & 6506 & 80 \\
\hline $\mathrm{H}(38 \mathrm{~B})$ & -1851 & 218 & 7289 & 80 \\
\hline $\mathrm{H}(38 \mathrm{C})$ & -1328 & -134 & 6688 & 80 \\
\hline $\mathrm{H}(37 \mathrm{D})$ & -621 & -379 & 7188 & 68 \\
\hline $\mathrm{H}(37 \mathrm{E})$ & -1155 & -49 & 7795 & 68 \\
\hline $\mathrm{H}(37 \mathrm{~F})$ & 171 & -204 & 8076 & 68 \\
\hline $\mathrm{H}(38 \mathrm{D})$ & -1345 & 1321 & 6537 & 74 \\
\hline $\mathrm{H}(38 \mathrm{E})$ & -2089 & 782 & 6809 & 74 \\
\hline $\mathrm{H}(38 \mathrm{~F})$ & -1503 & 571 & 6180 & 74 \\
\hline $\mathrm{H}(40 \mathrm{~A})$ & 685 & 846 & 8975 & 126 \\
\hline $\mathrm{H}(40 \mathrm{~B})$ & -648 & 838 & 8803 & 126 \\
\hline $\mathrm{H}(40 \mathrm{C})$ & 42 & 1532 & 9033 & 126 \\
\hline $\mathrm{H}(41 \mathrm{~A})$ & -1143 & 2125 & 7787 & 107 \\
\hline $\mathrm{H}(41 \mathrm{~B})$ & -1932 & 1469 & 7545 & 107 \\
\hline $\mathrm{H}(41 \mathrm{C})$ & -1353 & 1767 & 6948 & 107 \\
\hline $\mathrm{H}(31 \mathrm{~A})$ & 2945 & 223 & 7275 & 40 \\
\hline $\mathrm{H}(32 \mathrm{~A})$ & 4694 & -153 & 8112 & 46 \\
\hline
\end{tabular}




\begin{tabular}{lrrrr}
$\mathrm{H}(33)$ & 6311 & 503 & 8293 & 46 \\
$\mathrm{H}(34)$ & 6158 & 1531 & 7623 & 41 \\
$\mathrm{H}(35 \mathrm{~A})$ & 4399 & 1893 & 6773 & 40 \\
$\mathrm{H}(42 \mathrm{~A})$ & 2973 & 626 & 3704 & 66 \\
$\mathrm{H}(42 \mathrm{~B})$ & 3781 & 447 & 3211 & 66 \\
$\mathrm{H}(42 \mathrm{C})$ & 4021 & 1106 & 3763 & 66 \\
$\mathrm{H}(44)$ & 5324 & -381 & 3894 & 37 \\
$\mathrm{H}(45)$ & 6575 & -900 & 5111 & 38 \\
$\mathrm{H}(46)$ & -578 & 6304 & 39 \\
$\mathrm{H}(47)$ & 6338 & 165 & 6316 & 37 \\
$\mathrm{H}(48)$ & 4952 & 651 & 5166 & 38 \\
$\mathrm{H}(42 \mathrm{D})$ & 3785 & 389 & 3476 & 51 \\
$\mathrm{H}(42 \mathrm{E})$ & 3620 & 1039 & 3879 & 51 \\
$\mathrm{H}(42 \mathrm{~F})$ & 4349 & 849 & 4074 & 51 \\
$\mathrm{H}(44 \mathrm{~A})$ & 3237 & -210 & 3906 & 37 \\
$\mathrm{H}(45 \mathrm{~A})$ & 5327 & -918 & 4818 & 37 \\
$\mathrm{H}(46 \mathrm{~A})$ & 6430 & -829 & 6121 & 39 \\
$\mathrm{H}(47 \mathrm{~A})$ & 6475 & -32 & 6482 & 37 \\
$\mathrm{H}(48 \mathrm{~A})$ & 5318 & 618 & 5459 & 37 \\
\hline & 4027 & & &
\end{tabular}

${ }^{1}$ Laitar, D. S.; Müller, P.; Sadighi, J. P. J. Am. Chem. Soc. 2005, 127, 17196.

${ }^{2}$ Fristrup, P.; Le Quement, S.; Tanner, D.; Norrby, P.-O. Organometallics 2004, 23, 6160.

${ }^{3}$ Sheldrick, G. M. Acta Crystallogr. Sect. A 1990, 46, 467. 